University of Tennessee Health Science Center UTHSC Digital Commons

6-1999

\title{
Characterizing Erythrocyte Motions in Flowing Blood
}

Markos Leggas

University of Tennessee Health Science Center

Follow this and additional works at: https://dc.uthsc.edu/dissertations

Part of the Medical Cell Biology Commons

\section{Recommended Citation}

Leggas, Markos, "Characterizing Erythrocyte Motions in Flowing Blood" (1999). Theses and Dissertations (ETD). Paper 145. http://dx.doi.org/10.21007/etd.cghs.1999.0177.

This Thesis is brought to you for free and open access by the College of Graduate Health Sciences at UTHSC Digital Commons. It has been accepted for inclusion in Theses and Dissertations (ETD) by an authorized administrator of UTHSC Digital Commons. For more information, please contact jwelch30@uthsc.edu. 


\title{
Characterizing Erythrocyte Motions in Flowing Blood
}

\author{
Abstract \\ Characterizing the probabilistic motions of cells is a prerequisite for the development of a general model \\ of transport and surface deposition of white cells and platelets (WBC/P). These phenomena differ greatly \\ when red blood cells (RBC) are present at normal levels, but follow convective diffusion in dilute \\ suspensions. A critical need is to understand and characterize dispersion and diffusion as they apply to \\ red cells in suspension flow. The dispersive motions of \\ 0.5 -micrometer beads and fluorescently labeled human RBC flowing in dilute $(0.003 \%)$ and concentrated \\ (25\%) RBC suspensions, respectively, were characterized using fluorescence videomicroscopy methods, \\ and times for individual tracer particles to move fixed distances were measured. The particles were \\ tracked in the axial direction and in a moving reference frame. The \\ experimentally estimated effective diffusion coefficient of the particles was in good agreement with \\ published work (RBC $\sim 1 \times 10^{-8}$ and beads $\sim 4 \times 10^{-9}\left[\mathrm{~cm}^{2} / \mathrm{sec}\right]$ ). Using a continuous time random walk \\ model (CTRW) to characterize the particles' random motions in a shear field, the average time was plotted \\ versus the squared displacement and a power law fit exponent was used to quantitatively distinguish \\ between diffusion and dispersion. Values consistent with Brownian motion were found for the bead \\ suspensions and an anomalous diffusion was found for the RBC suspensions, which indicated that beads \\ random motions were diffusive and the RBC ones were dispersive. The methods developed in this work \\ could be used to study dispersion events at different lateral locations along the channel's height and \\ investigate the effects of flow parameters such as wall shear rate, hematocrit, and cell type.

\section{Document Type} \\ Thesis

\section{Degree Name} \\ Master of Science (MS)

\section{Program} \\ Biomedical Engineering

\section{Research Advisor} \\ Eugene C. Eckstein

\section{Keywords} \\ blood flow, diffusion, dispersion, rheology, suspension flow, random walk, first passage time

\section{Subject Categories} \\ Medical Cell Biology | Medical Sciences | Medicine and Health Sciences
}




\title{
CHARACTERIZING ERYTHROCYTE MOTIONS IN FLOWING BLOOD
}

\author{
A Thesis \\ Presented for \\ The Graduate Studies Council \\ The University of Tennessee, Memphis
}
In Partial Fulfillment
Of the Requirements for the Degree

\section{Master of Science}
In the Joint Graduate Program in Biomedical Engineering
From the University of Tennessee
and
The University of Memphis

By

\section{Markos Leggas}

June, 1999 
Copyright (C) Markos Leggas, 1999

All Rights Reserved 


\section{DEDICATION}

This thesis is dedicated to my family and friends. I thank them for their love and support through the time of my graduate study and research. 


\section{ACKNOWLEDGMENTS}

I would like to thank my mentor, Eugene C. Eckstein, Ph.D., for his guidance and support. I would also like to thank my other committee members, Mohammad F. Kiani, Ph.D., and R. Howard Berg, Ph.D. for their continued assistance throughout this study. In addition, I would like to thank Mr. Ajay Wagh (summer intern for 1998) and Mr. Baoshun Ma for their help in analyzing the videotaped images, and the other students in the lab for their assistance. I would also like to thank again Dr. Kiani and Dr. Eckstein for their friendship at a personal level. Finally, I want to acknowledge that this work was possible through funds from the J.R. Hyde Chair of Excellence and The University of Tennessee, Memphis Dean's Funds for Graduate Study. 


\begin{abstract}
Characterizing the probabilistic motions of cells is a prerequisite for the development of a general model of transport and surface deposition of white cells and platelets (WBC/P). These phenomena differ greatly when red blood cells (RBC) are present at normal levels, but follow convective diffusion in dilute suspensions. A critical need is to understand and characterize dispersion and diffusion as they apply to red cells in suspension flow. The dispersive motions of 0.5-micrometer beads and fluorescently labeled human RBC flowing in dilute $(0.003 \%)$ and concentrated (25\%) RBC suspensions, respectively, were characterized using fluorescence videomicroscopy methods, and times for individual tracer particles to move fixed distances were measured. The particles were tracked in the axial direction and in a moving reference frame. The experimentally estimated effective diffusion coefficient of the particles was in good agreement with published work $\left(\mathrm{RBC} \sim 1 \times 10^{-8}\right.$ and beads $\left.\sim 4 \times 10^{-9}\left[\mathrm{~cm}^{2} / \mathrm{sec}\right]\right)$. Using a continuous time random walk model (CTRW) to characterize the particles' random motions in a shear field, the average time was plotted versus the squared displacement and a power law fit exponent was used to quantitatively distinguish between diffusion and dispersion. Values consistent with Brownian motion were found for the bead suspensions and an anomalous diffusion was found for the RBC suspensions, which indicated that beads random motions were diffusive and the RBC ones were dispersive. The methods developed in this work could be used to study dispersion events at different lateral locations along the channel's height and investigate the effects of flow parameters such as wall shear rate, hematocrit, and cell type.
\end{abstract}




\section{Table of Contents}

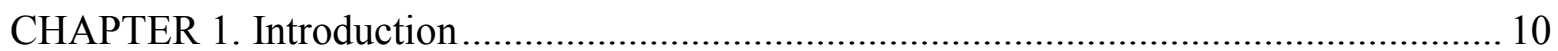

Definition of the Problem and Practical Significance .................................................. 10

A Summary of the Experimental Approach …………............................................. 12

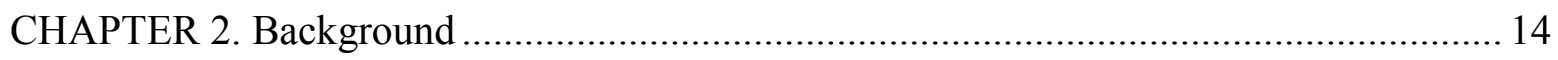

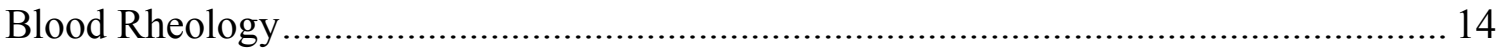

Flow and Transport Equations................................................................................ 15

Mechanisms of Lateral Transport............................................................................... 16

Previous Experimental Methods and Mathematical Models of Lateral Transport in

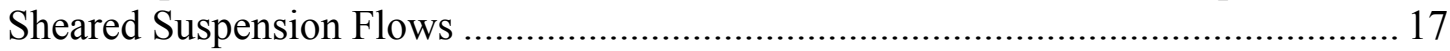

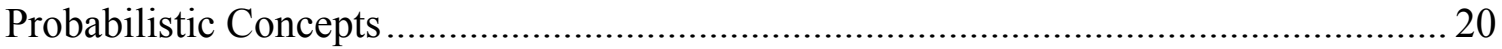

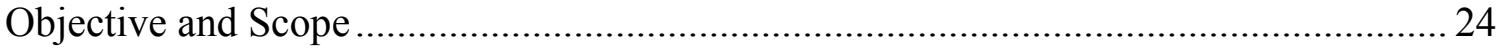

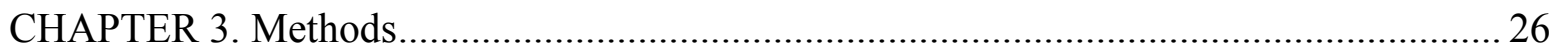

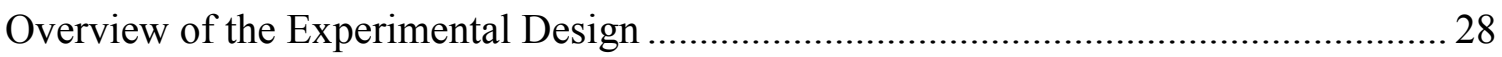

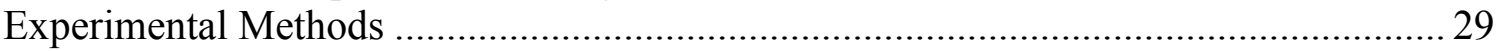

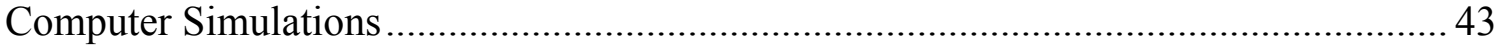

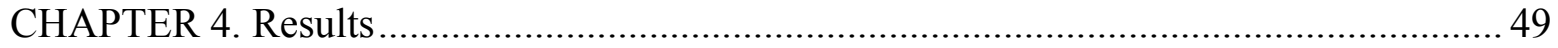

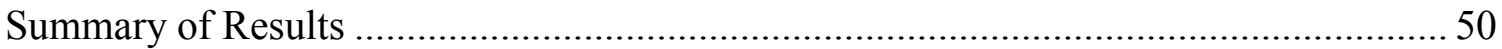

Estimation of the Effective Diffusion Coefficients ..................................................... 58

Index of Diffusion: Average FPT and Squared Axial Displacement Relationships ........ 60

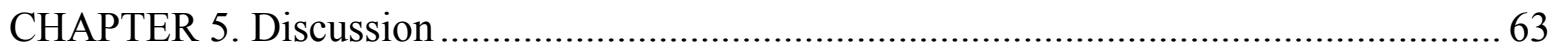

Comparisons of Results with Published Values................................................................6 66

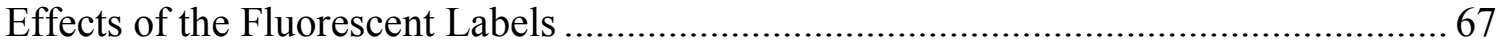

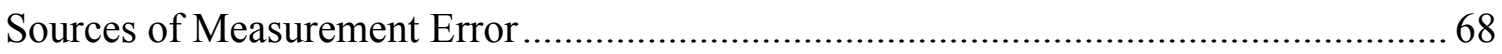

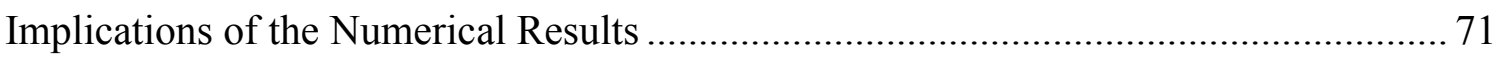

CHAPTER 6. Conclusions and Future Work................................................................. 72

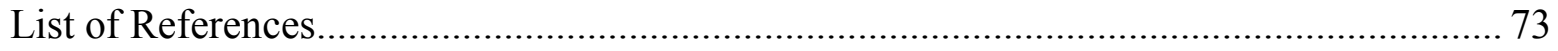

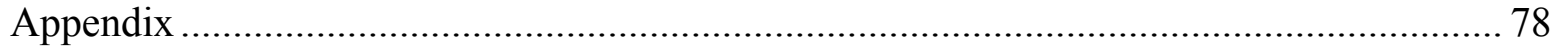

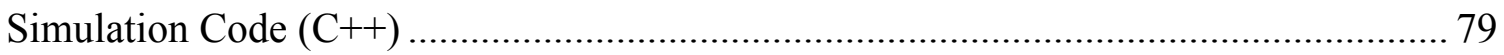

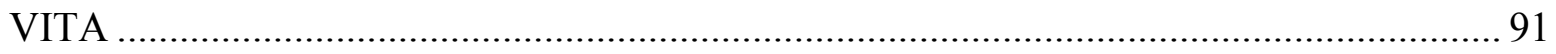




\section{List of Tables}

Table 3-1. The number of particles tracked during the experimental trials. .......................... 40

Table 3-2. The number of particles "tracked" with the numerical trials. ............................... 45

Table 4-1. Experimental results - Average first passage times for each trial. ....................... 52

Table 4-2. Numerical results - Average first passage times for each trial............................. 52

Table 4-3. Experimental results - Effective diffusion coefficients..................................... 59

Table 4-4. Numerical results - Effective diffusion coefficients........................................ 59

Table 4-5. Experimental Results - The character of diffusion........................................ 61

Table 4-6. Numerical results - The character of diffusion................................................ 62 


\section{List of Figures}

Figure 3-1. An outline of the experimental and numerical methods.................................. 27

Figure 3-2. Schematic representation of the flow apparatus............................................ 30

Figure 3-3. Schematic representations of the video and microscope (dotted line box)

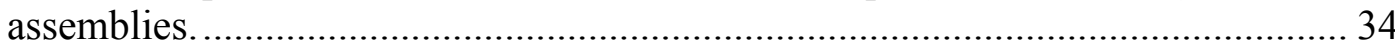

Figure 3-4. Schematic representation of the side view of the experiment. .............................. 36

Figure 3-5. Schematic representation of the first passage measurement for a p-type particle.... 39

Figure 3-6. Schematic of the flow profiles in the moving reference frame. .......................... 44

Figure 3-7. Flowchart of the simple random walk simulation for one particle...................... 47

Figure 3-8. Flowchart of the Kac walk simulation for one particle. .................................... 48

Figure 4-1. Average first passage time \pm standard deviation for combined experimental

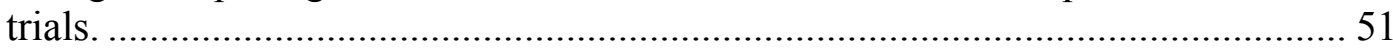

Figure 4-2. Average first passage time \pm standard deviation for combined numerical trials...... 52

Figure 4-3. Experimental result - Cumulative probability distributions for the first passage times of the bead trials............................................................................................... 53

Figure 4-4. Experimental results - Cumulative probability distributions for the first passage times of the RBC trials.

Figure 4-5. Cumulative probability distributions of the first passage times for combined KW numerical trials and $\mathrm{RBC}$ experimental trials.

Figure 4-6. Scaled cumulative probability distributions for all experimental and simulated trials at each fence distance..

Figure 4-7. Experimental results - Fractions of particles crossing a fence in the same direction as their initial direction of motion.

Figure 4-8. Experimental results - Fractions of p- and q-type particles crossing a fence in the direction of their initial motion.

Figure 4-9. Experimental results - Fractions of particles crossing a fence in the downstream direction

Figure 4-10. Comparison of numerical simulation $(\mathrm{KW})$ results to the experimental trials (RBC) 
Figure 4-11. Linear regression fits on the plot of the scaled average FPT vs. the squared axial displacement.

Figure 4-12. Experimental results - The character of diffusion was estimated by the exponent of the power law fit on the plot of the average FPT vs. the squared axial displacement..

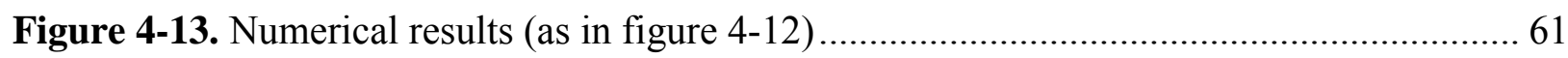

Figure 5-1. Box plots for the time measurement error................................................... 69 


\section{CHAPTER 1. INTRODUCTION}

In the work presented here, experimental methods and computer simulations that characterized the random motions of particles in suspension flows are described. The following items were the focus of the experimental efforts: 1) the effective diffusion coefficient of red blood cells (RBC) flowing through thin glass channels in a concentrated (25\%) suspension was estimated and compared to results of 0.46 micrometer diameter beads flowing in dilute suspensions $(0.003 \%), 2)$ the character of the diffusive motions was determined quantitatively, and 3) general observations regarding the cell and bead motions were made. The experimental work was performed within a theoretical probabilistic framework and the results were in agreement with previously published values. Numerical simulations based on random walk theories were also developed, and good agreement with experimental results was attained.

\section{Definition of the Problem and Practical Significance}

Blood is a biological suspension that consists of suspended particles and chemical solutes in water. Blood circulates throughout the body and has three general functions: 1) transportation, 2) regulation, and 3) protection. The functional effectiveness of blood depends on the interaction of its components and solutes with the vessel walls that contain them. Blood facilitates the transport of oxygen and removal of carbon dioxide and carries nutrients from the gastrointestinal system to the body cells while it takes wastes away. The tissue also supports the endocrine system by distributing hormones throughout the body. As a regulator, blood contains buffers to aid in maintaining constant $\mathrm{pH}$ levels and water to aid in regulating temperature. The protective function of blood is through the suspended cells. Among these, white blood cells (WBCs) protect against foreign microbes and toxins while platelets protect against blood loss through the clotting 
mechanism. Approximately $45 \%$ of the blood volume is comprised of formed elements or cells depending on gender and the individual. Ninety-nine percent of the cells are red blood cells, 0.7 percent are platelets, and the rest are white blood cells (40).

The flow-associated interaction among suspended particles has been shown to affect their transport properties (44). Clinical interest in the transport of platelets lies in their role in hemostasis and thrombosis and the interest in white blood cells is primarily for their important role in the immune response. The role of platelets is important in intravascular thrombosis because of the associated cardiovascular implications that may lead to death. Platelet interactions with artificial organs are also important because thrombus formation that may lead to malfunction of the implanted device or material. Finally, red cell interactions with implants, artificial organs, and biomedical devices are of importance for both pathological and functional reasons, namely hemolysis and gas exchange.

The functionality of both white blood cells and platelets depends on their ability to reach the vessel wall. Considering the relatively low numbers of white cells and platelets compared to red blood cells it is safe to postulate that the transport properties of the former would be affected by the dynamic motions of the later. Many studies have shown that there is an augmentation in lateral transport of solutes and particles in suspensions with red cells. The enhanced diffusion of platelets flowing through tubes when red blood cells are present has been investigated by Turitto (41), and the enhanced deposition of platelets on artificial surfaces has been attributed to the action of red cells in studies conducted by Brash (5). Additionally, the enhanced margination of white cells in the presence of red cells has been studied at low $(21 ; 30)$ and at high shear flows (29). The investigators concluded that the enhanced margination was due to the interaction of white cells with the red cells. 


\section{A Summary of the Experimental Approach}

In previous studies Goldsmith and coworkers traced particles along the tube axis using a traveling microscope apparatus. From the tracings of the particle positions in time it is obvious that while the particles always moved down the tube they simultaneously exhibited erratic lateral motions, hence, their axial velocity varied in time (20). In the studies by Goldsmith and coworkers and in the studies by others (6), the apparatus speed was adjusted so that the particle remained in view for long periods.

In this work, particles in suspensions that were flowing through glass channels were tracked using fluorescence videomicroscopy. Individual particle motions were tracked in a moving reference frame, which was constructed by moving the thin glass channel, mounted with its wide side flat on a moving microscope stage, in the opposite direction to the direction of the flow. The microscope stage traveled with a constant speed that corresponded to approximately the average speed of the flow; images collected by the objective as the stage moved were videotaped by a videocamera. In this frame, particles with a lesser speed than that of the microscope stage (i.e. particles flowing in streamlines closer to the wall) moved in one direction in the images, while particles with a higher speed than that of the microscope stage moved in the opposite direction (i.e. particles flowing in streamlines closer to the center). Fluctuations in the particle's lateral position appeared as changes in their speeds of axial motion and hence of their axial position because their average axial velocity was a function of their lateral position. Only those particles that appeared to change their axial directions of motion were tracked. This motion was believed to have been caused by the particle changing its lateral position from below the streamline corresponding to the microscope stage's speed to above or visa versa. In any case, all 
particles were tracked from a time when their initial axial speed was nearly zero as observed in the moving reference frame (MRF).

In the MRF, fluctuations in the particle's lateral position altered its axial speed and direction causing the particle to perform an apparent random walk in the axial direction and to eventually move out of sight on one or the other side of the video screen with varying speeds. Tracer particles often changed their axial direction or appeared to momentarily stop before they moved out of view. No significant motion was observed in the cross-lateral direction. Each particle was tracked until it reached a prescribed distance on one or the other side of its initial position for the first time and the interval of time needed to move this net distance was noted. All estimates of diffusion were based on the particles' axial displacement in the moving reference frame and the time intervals to reach those distances. 


\section{CHAPTER 2. BACKGROUND}

\section{Blood Rheology}

Extensive research efforts in the past decades have increased our understanding of the flow properties of blood suspensions. In the early part of this century, Robin Fåhraeus performed experiments that measured anomalous viscosity and concentration variations in vessels less than 300 micrometers in diameter $(3 ; 19)$. The concentration of erythrocytes ranges from lower near the wall to higher in the center and therefore the erythrocytes tend to have an average speed that is higher than the plasma. Consequently, vessel hematocrit decreases in the smaller branches of the vasculature. This phenomenon is known as the Fåhraeus effect. Another important observation made by Fåhraeus and Lindqvist was the decreased viscosity in smaller vessels. The Fåhraeus-Lindqvist effect can be explained by the marginal plasma layer that contains relatively few erythrocytes and acts as a lubricating zone by reducing the total flow resistance (8).

Other research efforts focused on the ability of erythrocytes to aggregate and form rouleaux - stacks of cells - at low shear rates (7). The aggregates tend to move toward the center and thus allow the formation of a plasma rich zone that has a lower viscosity. At higher flow rates however the rouleaux are exposed to higher shear stresses and are easily broken up. The flow velocity profile across the tube changes and may no longer be parabolic and it assumes a shape that is approaching plug flow or becomes blunted $(17 ; 18 ; 36)$. The decrease in viscosity at high shear rates may be partially explained by the mechanical properties of erythrocytes. These cells are able to deform in response to the shear stress and thus the viscosity is lower when compared to suspensions of rigid particles and hardened red cells (7). 
By studying the flow characteristics of erythrocytes and other particles, researchers have shown the dependence of blood rheology on shear rate, particle deformability, and diameter of the particle relative to the flow conduit. Extensive experimental work has shown that the radial migration is dependent on the particle deformability (20). Rigid spherical particles tend to flow with no radial migration while deformable ones tend to migrate toward the center. Goldsmith and associates showed that red cells exhibit random radial motions in the order of a cell diameter. These motions came about when the tracer cells collided with other suspended cells $(18 ; 20)$.

\section{Flow and Transport Equations}

In the experiments presented here, the traditional treatment would represent the axial flow as occurring between two flat plates (4) and simplify the Navier - Stokes equations to the Stokes equation

$$
\frac{\partial \mathrm{p}}{\partial \mathrm{x}}=\frac{1}{\mu} \frac{\partial^{2} \mathrm{u}}{\partial \mathrm{y}^{2}}
$$

where $\mathrm{p}$ is the pressure, $\mathrm{x}$ is the axial direction, $\mathrm{y}$ is the lateral direction, and $\mu$ is the viscosity. Equation 2-1 can be solved with the appropriate boundary conditions to give the Poiseuille formula.

$$
\mathrm{u}(\mathrm{y})=\frac{3}{2} \overline{\mathrm{u}}\left[1-\frac{\mathrm{y}^{2}}{\mathrm{Y}^{2}}\right]
$$

Equation 2-2

Where $\overline{\mathrm{u}}$ is the average velocity given by the ratio of the flowrate and the cross-sectional area of the channel, $\mathrm{Y}$ is the channel's half height and y is the lateral (height) position away from the center. Equation 2-2 is valid for laminar, incompressible flow of liquids. 
The convective diffusion equation that can represent mixing motions in this simple system is given by equation $2-3$.

$$
u(y) \frac{\partial c}{\partial x}=D \frac{\partial^{2} c}{\partial y^{2}} \quad \text { Equation 2-3 }
$$

where $\mathrm{c}$ is the concentration, and D is the diffusion coefficient. Using dimensional analysis, the ratio of the convective term on the left side of equation 2-3 to the diffusion term on the right gives the non-dimensional Peclet number that determines the governing mode of transport (i.e. diffusive or convective).

$$
\mathrm{Pe}=\frac{\mathrm{Y} \overline{\mathrm{u}}}{\mathrm{D}} \quad \text { Equation 2-4 }
$$

\section{Mechanisms of Lateral Transport}

In dilute suspensions, particles with sizes ranging from the submicron (colloidal) (6) and up to the platelet (41) size ( $\sim 2$ micrometers) are subject to Brownian motion. Brownian motions are random motions caused by the imparted energy of fluid molecules on the suspended particle. In concentrated suspensions however, the interaction among particles dominates any particlefluid interaction and gives rise to an indeterminate erratic path.

The diffusive phenomena in suspensions are currently classified in three ways: 1) selfdiffusion; b) diffusion from regions of high concentration to low; and 3) diffusion from regions of high shear to low (2). Self-diffusion is due to particle - particle interactions and can be measured in Couette devices $(12 ; 27)$ where the shear and concentration profiles are constant (4). Specific particle interaction mechanisms that give rise to augmented net lateral transport include: 1) particle rotations that cause adjacent fluid mixing, 2) shear-induced collisions that enhance 
diffusion, and 3) in the case of deformable particles such as red cells, internal fluid mixing that causes erratic motions (44 (references therein)).

\section{Previous Experimental Methods and Mathematical Models of Lateral Transport in Sheared Suspension Flows}

Using concepts from Taylor dispersion $(11 ; 38 ; 39)$, Turitto and coworkers measured the diffusion coefficient of platelets flowing through tubes in suspensions of variable hematocrits and shears. The method however did not prove very sensitive to changes in hematocrit and shear. Furthermore, the method required extremely long tubes and the observations were subject to scaling conditions (e.g. $t>R^{2} / D$ and 7.2(LD/R $R^{2}>>1$, where $D$ is the diffusion coefficient, $R$ is the tube radius, $\mathrm{L}$ is the length of the tube and $\mathrm{v}$ is the average velocity) (41).

Eckstein and Bailey measured the self-diffusion of spheres and discs flowing in a Couette device over a range of concentrations and shears (12). In their experiments a radioactive particle traveled around the Couette device and the change in lateral position (the $\mathrm{y}$ - direction) was measured along with the time required to complete the travel. The random particle motions were treated as a random walk and the diffusion coefficient was estimated with equation 2-5.

$$
\mathrm{D}=\frac{1}{2 \mathrm{~N}} \sum_{1}^{\mathrm{N}} \frac{\left(\Delta \mathrm{y}_{\mathrm{i}}\right)^{2}}{\Delta \mathrm{t}_{\mathrm{i}}} \quad \text { Equation 2-5 }
$$

where $\Delta \mathrm{y}_{\mathrm{i}}$ was the lateral displacement and $\Delta \mathrm{t}_{\mathrm{i}}$ was the time between measurements of the lateral position, $\mathrm{y}_{\mathrm{i}}$, in the Couette device. Using dimensional analysis, the researchers established relationships relating the diffusion coefficient to the particle size and shear rate as a function of concentration. Interested readers are referred to the original article for details. 
The diffusion coefficient of red cells has also been estimated using equation 2-5 by measuring the lateral displacement of red cell tracers flowing in tubes (20). The authors reported effective diffusion values in the order of $10^{-8} \mathrm{~cm}^{2} / \mathrm{sec}$. In that work the particle was tracked by a translating microscope apparatus that followed the particle motions in a stationary tube.

Leighton and Acrivos also measured the self-diffusion of large particles in a Couette device and developed expressions depicting the dependence of diffusion on shear rate (27). Their model is currently used by others in the analysis of flow in tubes and channels (32), but it is not clear that it is effective in the center region of tube and channel flows where presumably the shear rate approaches zero. Furthermore, analysis performed by Nott and Brady (31) shows that the solution of the convective diffusion equation with the use of a shear dependent diffusion equation would give an abnormal concentration profile - cusp - in the center region. This result is also in contradiction with the experimentally determined concentration profiles presented by Koh and others $(1 ; 26 ; 37)$. Nonetheless, their model is very useful in calculating diffusion coefficients and presumably less prone to errors.

In their analysis, Leighton and Acrivos assumed that the distribution of the random displacements was Gaussian, and developed an expression for the calculation of diffusion based only on the successive times to complete a trip - distance $x_{0}-$ around the Couette device in which the suspension was being sheared with shear rate $\gamma$. For $N+1$ measurements the observed diffusion coefficient can be estimated with equation 2-6

$$
\mathrm{D}_{\mathrm{obs}}=\frac{1}{2 \mathrm{~N}} \sum_{1}^{\mathrm{N}} \alpha_{\mathrm{i}}^{2}
$$

where, $\alpha_{\mathrm{i}}^{2}=\frac{\left(\mathrm{t}_{\mathrm{i}}-\mathrm{t}_{\mathrm{i}-1}\right)^{2}}{(1 / 3) \mathrm{t}_{\mathrm{i}}^{2} \mathrm{t}_{\mathrm{i}-1}^{2}\left(\mathrm{t}_{\mathrm{i}}+\mathrm{t}_{\mathrm{i}+1}\right)\left(\gamma / \mathrm{x}_{\mathrm{o}}\right)^{2}}$. 
The variables $t_{i-1}, t_{i}$, and $t_{i+1}$ are the consecutive times to travel a distance $x_{0}$.

In an analysis presented by Foister and van de Ven, a relationship was developed for the mean squared displacement of Brownian particles flowing in dilute sheared flow (14).

$$
<\mathrm{x}^{2}>=\mathrm{D}\left(2 \mathrm{t}+\mathrm{kt}^{3}\right)
$$

\section{Equation 2-7}

where the $\mathrm{D}$ is the diffusion coefficient and $\mathrm{k}$ is a constant relating the maximum velocity, $\mathrm{V}_{\max }$, the tube radius $R$, and the initial lateral position of the particle $r_{o}$ as, $k=\left(\frac{V_{\max }}{R^{2}}\right)^{2} r_{o}^{2}$.

Their model assumed convection and diffusion as the modes of transport, which gives a Gaussian distribution of the displacements, and by the calculation of the second moment, the variance of the displacements, using the Langevin equation. For a detailed analysis of the derivation of transport equations through the Langevin and Fokker - Plank equations the reader is referred to the text by Gardiner (15).

Foister and van de Ven partially tested their model with limited experiments, but it was later tested by experimentally measuring the diffusion of small bead ( 0.5 micrometers) doublets in shear flow (6). The experiments estimated the particle diffusion coefficient in the order of $3 \times 10^{-9} \mathrm{~cm}^{2} / \mathrm{sec}$ and the mean squared displacement of the particles was related to time with an exponent of 3.19 , (i.e. $\left\langle\mathrm{x}^{2}\right\rangle \sim \mathrm{t}^{3.19}$ ). The estimated value of diffusion was in the order of the value calculated by using the Stokes-Einstein formula.

In their experiments, Cerda and van de Ven traced particles by using an apparatus that consisted of a moving - tube flow - assembly. The particles were always kept in a fixed position on the video screen by using a feedback mechanism that controlled the speed of the moving apparatus. The distance traveled and time were displayed on the video monitor. To calculate the 
displacement due to fluctuations in the particle's lateral position, they subtracted the displacement that the particle was expected to travel if it had remained in the same initial lateral position (assuming infinite Peclet number) from the total displacement that was displayed on the video screen - due to both convection and diffusion. The initial lateral position of particles was in the range of 50-80 micrometers from the wall of a tube with diameter of 190-micrometers. The shear rates that the beads were subjected to varied from 0.5 to $1.2 \mathrm{sec}^{-1}$.

A mathematical model developed by Compte used continuous time random walk theory to develop expressions relating the mean squared displacement to time for the cases of diffusion, subdiffusion, and superdiffusion. Continuous time random walks have proven to be useful tools in describing diffusion in disordered media. The model used Levy distributions to describe the displacement of random walkers and in the case of Brownian motion the solution obtained was equation 2-7. Compte developed additional expressions to treat anomalous diffusion and found that values of the time exponent varied from 3 to the degree that the motions varied from the Brownian ideal (10). Compte showed that for Brownian motion the relationship of the squared mean displacement and time were given by $\left\langle\mathrm{x}^{2}\right\rangle \sim \mathrm{t}^{3}$, and for the cases of superdiffusion and subdiffusion the exponent was less than 3 or higher than 3 , respectively. In the context of this work, superdiffusion is equivalent to dispersion.

\section{Probabilistic Concepts}

Several concepts commonly treated in probability theory, but also often appearing in textbooks on physics or applied science, are reviewed to provide a common language for the remainder of the thesis. The ideas here are basic and fundamental, but quite deep in the mathematical connections that exist between the discrete forms that are discussed and the 
continuous mathematics of diffusion, which most of the prior discussion has assumed. That depth is not covered.

\section{Brownian Motion}

The Dutch physician Jan Ingenhausz first observed the phenomenon of Brownian motion in 1785 while he was looking at powdered charcoal on an alcohol surface. However, the phenomenon was named after the Scottish botanist Robert Brown, who in 1828 published his investigation of the random motions of pollen, dust, and soot on a water surface (25). Einstein was the first to explain the Brownian motion in physical terms. He proposed that the random thermal motions of fluid molecules striking the microscopic particles were the cause of the particles' erratic motions that appeared as a random walk (25). Perrin used Einstein's theoretical model to directly measure Brownian motion and calculate Avogadro's number (23). The formula most often used to calculate the diffusion of small particles suspended in a stagnant fluid is the Stokes - Einstein formula, $\mathrm{D}=\mathrm{kT} / 6 \pi \mu \alpha$, where $\mathrm{k}$ is Boltzman's constant, and $\alpha$ is the particle radius $(15)$.

\section{Random Walks}

Random walks are probabilistic constructs that can be used to model continuous phenomena in discrete temporal and spatial terms. The associated mathematical equivalents for the continuous representations of events in time and space are usually partial differential equations with specific solutions obtained with the use of initial and boundary conditions. In cases where those conditions are not known or the equations are difficult to solve, it is more convenient to model events as random walks. 


\section{Simple Random Walk}

Random walks may be used to model the erratic motions of particles. One of the first known random walk models is the Drunkard's walk where in one-dimensional coordinates the walker moves at fixed time intervals to the left or to the right with equal probability. After each step the walker must decide which way he should go and since the probability to move to either direction is equal to $1 / 2$, the direction of the new step is independent of the direction of the previous step(s). In probability terms, this property makes the simple random walk (SRW) a first order Markov process. It should be noted that SRW steps modeled as discrete steps on a lattice or as random variables with a Gaussian distribution lead to the same results (43). In his book Aspects and Applications of the Random Walk, Weiss explains how the convective diffusion equation can be derived from the probabilistic parameters associated with the displacement of random walkers (e.g. the probability density of the displacement, and the means squared displacement).

\section{$\underline{\text { Persistent Random Walk }}$}

If the random walker in the previous section has a tendency to keep moving in the same direction, the random walk is called a persistent random walk. In that case, the walker has to also consider his direction of motion when he takes the next step. Once the walker changes direction the probability to keep moving in the new direction is higher than the probability of reversing directions. The decision process requires two pieces of information, the current position, and previous direction in order to generate a new step. In probability terms, this type of random walk is known as a second order Markov process, and the derivation of the associated differential equation leads to a telegrapher's equation, which is an interpolation between a wave and a diffusion equation. This equation can then lead to a wave or a diffusion equation in limiting 
cases (43). Kac also derived the telegrapher's equation for a persistent random walk (KW), whose steps in each direction followed a Poisson distribution (24). This type of walk was used in a simulation developed in this work and it has been called the Kac Walk.

\section{Continuous Time Random Walks (CTRW)}

Continuous time random walks are a way to incorporate variable time intervals in the random walk model by treating time as a random variable and not as a fixed interval between spatial steps as in the previous Markovian examples. The random walk can be on a lattice or have variable length steps. The times between each step can be considered as random variables that are described by a stable or Levy distribution (i.e. they have an infinite mean) (10; 43). This type of random walk has been proven very useful in describing events in disordered media. Both the Kac and simple random walks can be viewed as special cases of the CTRW.

\section{First Passage Measurements}

A useful tool in the random walk and Markov processes theory is the first passage time measurement. A brief explanation of this concept is provided in this section but the associated analysis is omitted. Full descriptions with mathematical analysis are provided in probability literature $(16 ; 28 ; 43)$.

Assume a system can be characterized by many states $\mathrm{s}_{1}, \mathrm{~s}_{2}, \mathrm{~s}_{3} \ldots$ and suppose that the state is initially at $\mathrm{s}_{0}$. The system may achieve the different states with time. The first passage time measurement is the time which the system has made a transition from state $\mathrm{s}_{0}$ to a new state, e.g. $\mathrm{s}_{1}$, for the first time. The first passage time is a random variable with a characteristic distribution. The calculation of moments that characterize the distribution depends on the nature 
of the random variable. Results can be derived for continuous and discrete random processes and in the context of random walks in one or more dimensions (28).

The "first passage time measurement" is obviously a structured analytical device. There are always defined states. The measurement starts when the system is at one state and stops when the system reaches a new state and the states are independent of the random process (at least in the context of this work). In this work, the first passage time has been used more for the structure that it offers rather than for extensive analysis of the first passage time distributions. The later however is subject to future work since the first passage time is a representation of the underlying random process (16). Again, in the context of the present work the states were defined in terms of the axial position of the tracer particles.

\section{Objective and Scope}

Cells flowing in biological suspensions exhibit enhanced diffusion due to the dynamic interactions of red cells. In this study, the erratic motions of red cells were characterized by measuring their diffusion coefficients, and determining the nature of their diffusive behavior by developing expressions for their squared axial displacement in time.

As noted earlier, studies in Couette devices have been used to characterize the random motions of particles. In those studies, mathematical expressions that related the diffusion coefficients to particle size, shear rate, and concentration were developed and were in the form of a non-dimensional diffusion coefficient, $\hat{\mathrm{D}}(\phi)=\frac{\mathrm{D}}{\gamma \alpha^{2}}$, where $\mathrm{D}$ is the diffusion coefficient, $\gamma$ is the shear rate, and $\alpha$ is the particle's radius $(12 ; 27)$. In this study the intent was to develop an experimental method that could be used to systematically characterize particle motions in narrow 
lateral regions across the height of glass channels. In other words, the methods were intended to make local measurements over the range of concentration and shear gradients in channel flows.

Three trials with blood cell suspensions, whose concentration was $25 \%$ by volume in a buffered albumin solution, were conducted. The flowrate was the same for each experiment, and measurements were made in the same lateral region, approximately $1 / 2$ the distance between the channel wall and the center-plane of a $\sim 50$ micrometer thick channel. The wall shear rate was approximately $185 \mathrm{sec}^{-1}$, assuming Poiseuille flow between two flat plates. Three additional experiments studied the motions of 0.46 -micrometer diameter polystyrene microspheres, which were conducted under the same flow conditions as above in dilute bead suspensions $(0.003 \%)$. These results of the bead experiments served as "quality" control experiments and were compared to published findings obtained by similar methods.

A secondary objective was to numerically model particle motions. The goal was to simulate motions in two directions, axial and lateral, where the motions could be modeled by convection and diffusion, respectively. The convective motions were treated as Poiseuille flow and the lateral motions as random walks. The results between a simple random walk and a Kac walk were compared. 


\section{CHAPTER 3. METHODS}

Techniques and concepts from many areas were used to describe and analyze particle behavior in suspension flows. The methods consisted of standard protocols for microcirculation and physical or colloidal suspensions, and the analysis was based on probabilistic and mathematical models.

The effective diffusion coefficients of commercially available 0.46 -micrometer fluorescent beads and fluorescently labeled human red cells suspended in buffered albumin were determined. Measurements were performed on a dilute bead suspension ( $0.003 \%$ beads) and a concentrated ( $25 \%$ red cells) erythrocyte suspension. Particles were tracked in a moving reference frame where initial and ending states or conditions could be easily defined and imposed repetitively. The data provided a qualitative picture of the RBC motions and a quantitative way to measure the lateral transport of particles in flowing suspensions.

Particle motions were simulated numerically in two dimensions. The axial motions were modeled as convective (Poiseuille flow) and the lateral motions as random walks. Two different random walks were used to simulate the lateral motions. Results of these numerical simulations provided hints about the underlying random processes that may be involved in particle transport.

An overview of the work is shown in figure 3-1. Details about specific topics in the boxes are discussed in the next sections. 


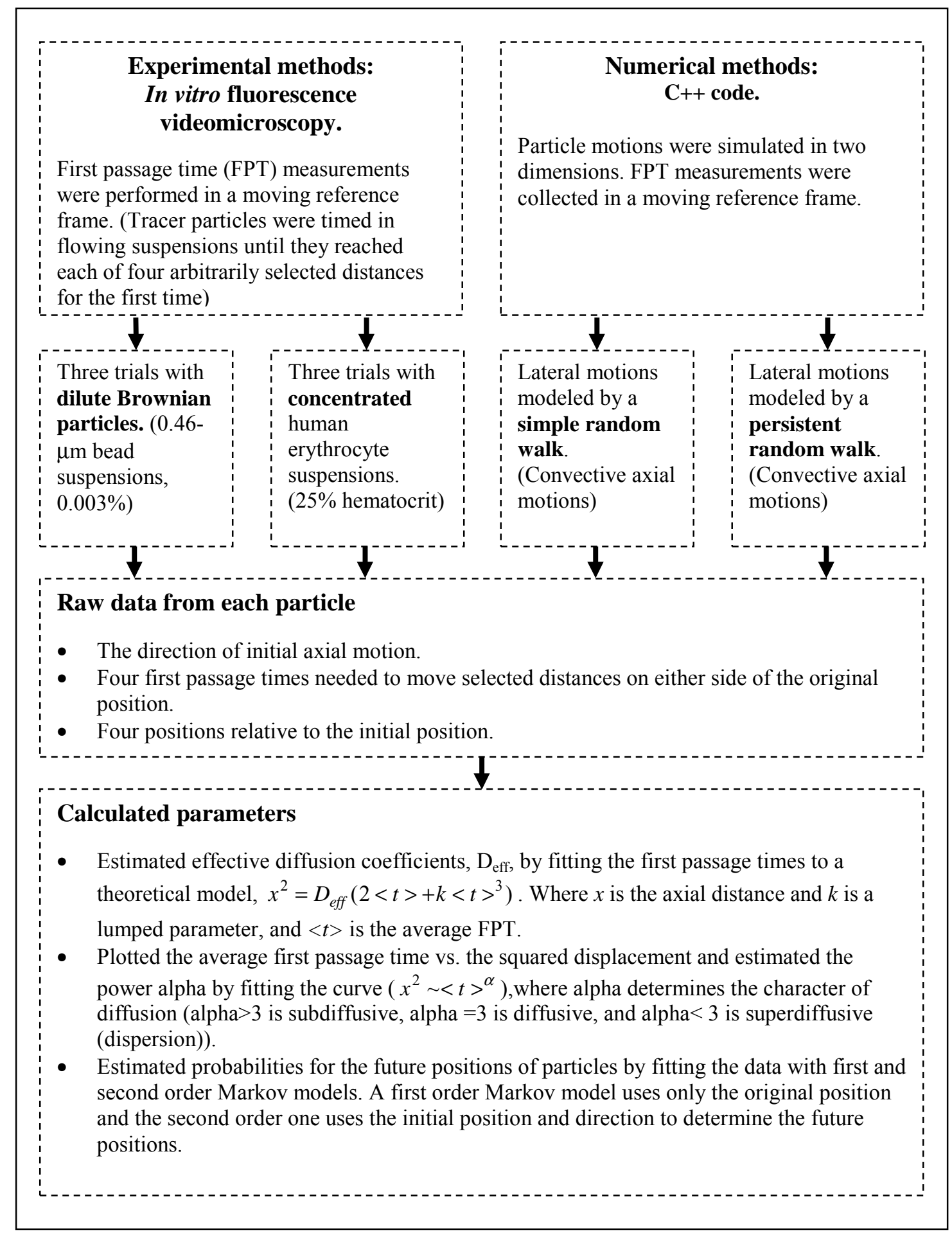

Figure 3-1. An outline of the experimental and numerical methods. 


\section{Overview of the Experimental Design}

The suspension flowed in a thin rectangular channel that was mounted on a motorized microscope stage that could move at high speeds. The moving reference frame was obtained by recording particle motions while the microscope stage moved in the opposite direction of the flow in the mounted channel. The narrow dimension of the channel, which would more directly show differences of speed due to particle position within the channel, was aligned with the focal direction of the microscope. In the video image, the flow axis was horizontal and the channel width was vertical (i.e. the height of the channel and the lateral flow velocity profile were perpendicular to the video image). Fluorescence microscopy enabled the observer to see only the small fraction of fluorescently labeled particles and select an even smaller number of particles for analysis. The camera signal from the experiments was recorded on a SVHS videocassette recorder with a time stamp applied to each video frame. This simplified analysis of the video images and prevented measurement errors associated with dropped frames. The measurements on images were done with analysis software that allowed some of the procedures to be automated with macro programming.

Particles were selected for analysis if they reversed their axial motion. For each particle the following data were recorded: 1) the initial position (centroid), 2) the initial time displayed on the video frame, 3 ) the initial axial direction as it was inferred by two consecutive video frames, and 4) the time displayed when the particle first crossed each of four pairs of boundaries located on either side of its initial position, and 5) the centroid of the particle as it crossed over each boundary. 


\section{Experimental Methods}

\section{Flow Apparatus}

A long section of a thin rectangular glass channel (Friedrich \& Dimmock, NJ) was used for all the experiments $(25 \mathrm{~cm} \times 54 \mu \mathrm{m} \times 556 \mu \mathrm{m}$ with $50-\mu \mathrm{m}$ walls $)$. The rectangular channel shape was chosen to avoid optical distortion problems associated with the curvature of glass tubes when these are observed via microscopy. The height-to-width ratio was chosen so that the average cross-lateral flow velocity profile would be nearly identical across the majority of the channel's width. Each end of the channel was carefully inserted in polyethylene tubing (PE-60) to allow easy connections with other pieces of the flow apparatus with the length of the tubing measuring $30 \mathrm{~cm}$ on one side and $3 \mathrm{~cm}$ on the other. The glass was secured in the tubing with epoxy glue and cured for a minimum of 24 hours to assure a strong, leak-free connection.

In preparation for the experiment the channel assembly described above was washed with $10 \mathrm{ml}$ of ethanol, $20 \mathrm{ml}$ of water, and $30 \mathrm{ml}$ of HEPES buffer with $2.5 \%$ BSA in that order. Following the experiment the channel was washed with HEPES buffer, water, and ethanol.

The channel assembly was carefully aligned and its position secured on the motorized microscope stage. Figure 3-2 shows a schematic representation of the microscope stage and the channel assembly. The channel was positioned so that its wide side was flat on the surface of the stage. A 5-ml cylindrical plastic container was secured to the top of the stage (MAC 2000 controller and 3" x 4" motorized stage, Ludl Electronic Products, Hawthorne, NY) and used as a suspension reservoir. A hole was drilled and threaded near the base of the cylinder. A small plastic connector with matching threads was used to connect the short tube end of the channel to 


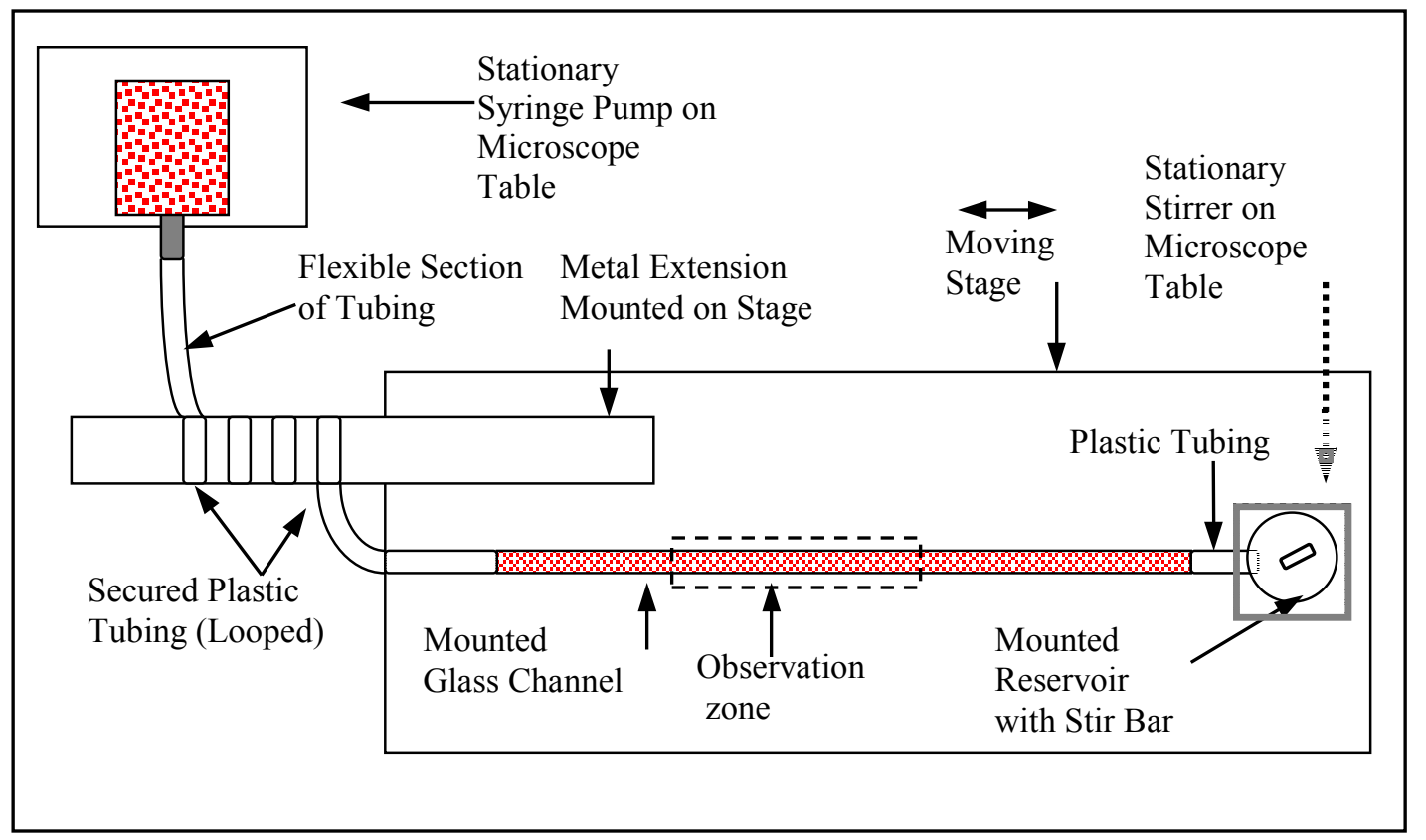

Figure 3-2. Schematic representation of the flow apparatus. Everything was located on the top of a vibration isolation table. The suspensions were withdrawn through the channel by the syringe pump.

the plastic container. A small amount of silicone vacuum grease was applied around each connection during the experiments to ensure the integrity of the seal. The long tubing at the other end of the channel assembly was wrapped around a metal extension of the stage and connected to a syringe located on a pump (Harvard Apparatus Pump 22, Harvard Apparatus Co., South Natick, MA). The syringe pump was stationed on the microscope table and used to withdraw the suspension from the reservoir. The plastic section of tubing from the metal stage extension to the syringe was kept relatively straight and in a lower position relative to the channel, allowing it to freely move with the stage motion. A simpler system was used with the bead suspensions, which were withdrawn using a miniature BAS Bee syringe pump (Bioanalytical Systems Inc., Lafayette, IN) that was located on the travelling microscope stage. 


\section{Suspension Methods}

\section{$\underline{\text { Red Cell Separation and Labeling }}$}

Following protocols approved by a Human Subjects Committee, whole blood was taken from healthy adult volunteers via standard phlebotomy of the median cubital vein. Samples were collected in three 5-ml sterile vacuum containers (Becton Dickinson, Franklin Lakes, NJ) containing an anticoagulant ( $\mathrm{K}_{3}$ EDTA). Subsequently, the contents of each tube of blood were transferred to a $15-\mathrm{ml}$ polyethylene centrifuge tube and diluted with $9 \mathrm{ml}$ of cold $\left(4{ }^{\circ} \mathrm{C}\right) \mathrm{HEPES}$ buffer solution and $0.2 \mathrm{ml}$ of anticoagulant ACD-A. An aliquot of ACD-A solution was added each time the cells were re-suspended. The suspension was centrifuged at 900 revolutions per minute for 5 minutes (IEC Centra-8R Centrifuge, International Equipment Co., USA.) The erythrocytes were isolated by removing the plasma and the buffy coat and the packed red cells were re-suspended in buffer. The washing procedure was repeated twice more. A 1-ml portion of packed cells was suspended in HEPES buffer and was set aside for the fluorescent labeling procedure. The rest of the packed cells were washed with 2.5\% Bovine Serum Albumin/HEPES buffer. The hematocrit was adjusted to $25 \%(\mathrm{v} / \mathrm{v})$ with the buffered albumin solution. Hematocrit values were checked with a microcentrifuge (Drucker Co., Model 412, Hialeah, FL). The suspension was refrigerated until use.

A double labeling procedure was developed to enhance the brightness of the red cells in the images. The first label was a membrane permeable dye that underwent chemical modification once inside the cell. The modified molecules could not penetrate the cell membrane and remain dissolved in the cytoplasm. The second label was a lipophilic dye that is incorporated into the cell membrane. 
Red blood cells for staining were prepared using the centrifuge washing procedure discussed above. In all the washing procedures, a $0.1-\mathrm{ml}$ portion of anticoagulant ACD-A was added each time the cells were re-suspended in buffer solution. A 1-ml portion of packed cells was suspended in $10 \mathrm{ml}$ of buffer. The suspension was mixed with 12 microliters of 5-(and-6)(((4-chloromethyl) benzoyl)amino)tetramethylrhodamine stock (1mg in 200 microliters of DMSO) (Cell Tracker Orange $®$, Molecular Probes, C-2927, maximum excitation/emission $540 / 566 \mathrm{~nm}$, Eugene, OR), and it was gently stirred with a magnetic stirrer for one hour in the dark. The cells were washed twice with HEPES buffer (34) and re-suspended in $10 \mathrm{ml}$ of buffer and $0.1 \mathrm{ml}$ of DiI fluorescent dye stock solution (5mg in $10 \mathrm{ml}$ of absolute ethanol) (Molecular Probes, D-282, maximum excitation/emission 549/565 nm, Eugene, OR) (35; 42). The suspension was gently stirred using a magnetic stirrer for one hour in the dark. The cells were washed three times with HEPES buffer and a 10\% suspension of labeled RBCs was prepared in HEPES buffer with $2.5 \%$ BSA. The sample was shielded from light and refrigerated until ready to use.

The blood suspension was prepared by mixing $3 \mathrm{ml}$ of the $25 \%$ erythrocyte suspension with 10 microliters of the $10 \%$ labeled erythrocyte suspension. The suspension was placed in a plastic container that was used as the reservoir. The resulting labeled cell fraction was approximately $0.03 \%$.

\section{Preparation of the Bead Suspension}

A $2 \%$ commercially available suspension of 0.46 micrometer diameter fluorescent polystyrene beads (Molecular Probes, F-8813, maximum excitation/emission 505/515 nm, specific gravity $=1.05$, Eugene, $\mathrm{OR}$ ) was sonicated for three minutes. All the preparations were processed in containers that protected the fluorescent materials from light. Three microlitter of 
the bead suspension were added to $25 \mathrm{ml}$ of $2.5 \% \mathrm{BSA} / \mathrm{HEPES}$ buffer, and the suspension was mixed and then sonicated for 2 minutes. After standing for 1.5 hours, the suspension was again mixed and then sonicated for 2 minutes. A $5-\mathrm{ml}$ solution of $2.6 \% \mathrm{Na}_{2} \mathrm{SO}_{4}(\mathrm{w} / \mathrm{v})$ with specific gravity of 1.02 at $25{ }^{\circ} \mathrm{C}$ was placed in the reservoir, and 60 microlitters of the buffered bead suspension were added. The final bead fraction was less than $0.003 \%$.

\section{Microscopy and Experiment Recordings}

\section{$\underline{\text { Hardware }}$}

The particle motions from each experiment were recorded using fluorescence videomicroscopy techniques. The connections between microscope and video recording assemblies are shown in figure 3-3. The appropriate light filter was used to control the wavelength of the light sensed by the camera. For the red cell trials, the filter characteristics were as follows: excitation, $535 \mathrm{~nm}$; dichroic, $575 \mathrm{~nm}$; and emission, $590 \mathrm{~nm}$ (Omega Optical, Brattleboro, VT). For the fluorescent bead trials, the filter characteristics were as follows: excitation, $475 \mathrm{~nm}$; dichroic, $505 \mathrm{~nm}$; and emission, $535 \mathrm{~nm}$ (Omega Optical, Brattleboro, VT). A Nikon industrial grade microscope body with a trans-illumination (Opti-Quip 75W xenon light source Model No. 1500, Highland Hills, NY) and epi-illumination (Nikon 150W mercury lamp Model No. HB-10101AAF, Japan) light sources was used. Preliminary set-up procedures were done in trans-illumination mode with a $4 \mathrm{x}$ or 10x objective. Recordings of particle motions were made in fluorescence mode with a 10x objective ( 0.25 numerical aperture). The primary camera assembly consisted of Dage-MTI intensified CCD camera and controller (Dage-MTI CCD CCD72, GenIISys 104722-01, Michigan City, IN). A second Hamamatsu CCD camera was also 


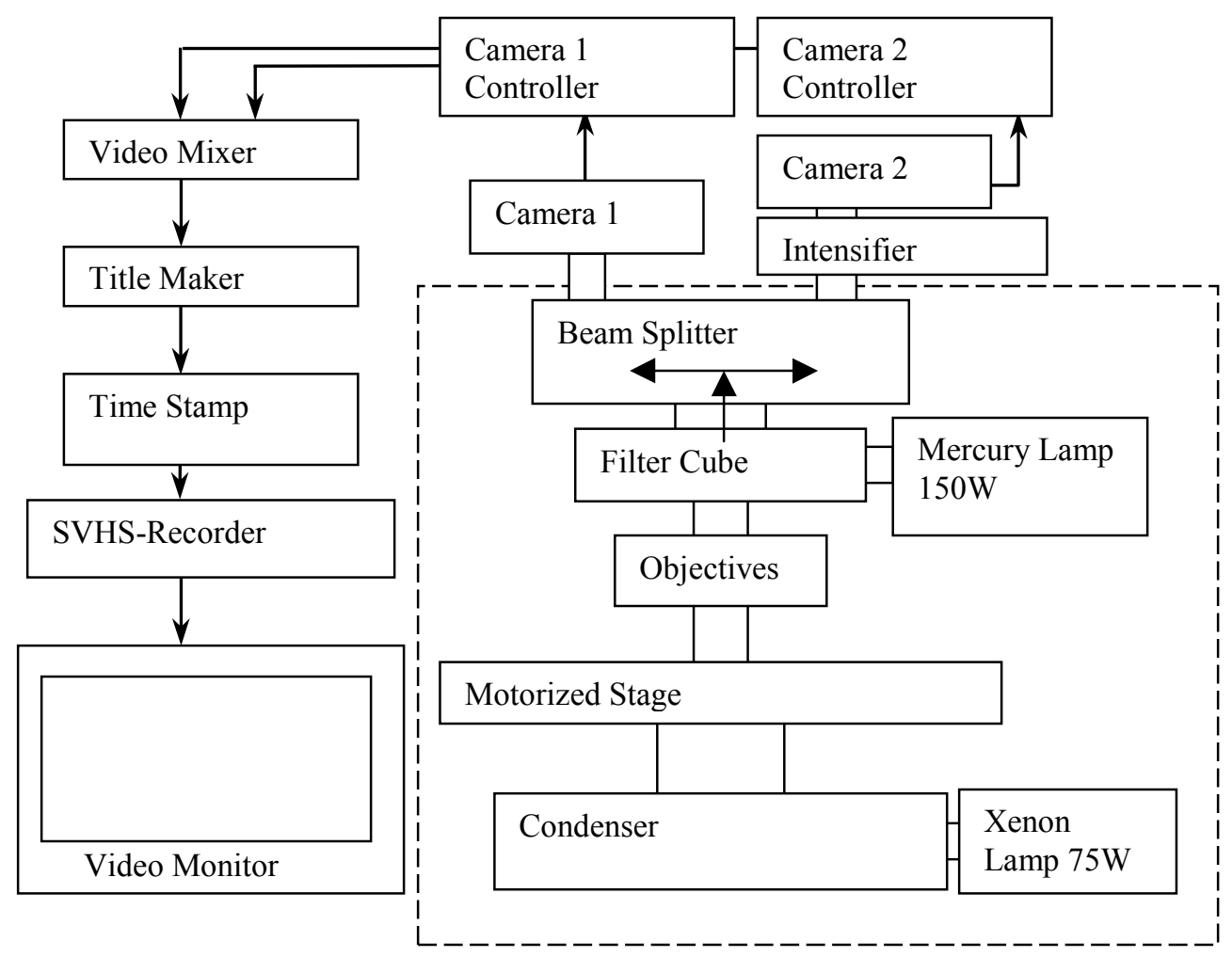

Figure 3-3. Schematic representations of the video and microscope (dotted line box) assemblies.

mounted, and was sometimes used for stage-speed calibration and channel-dimension measurements (Hamamatsu C5985, Japan).

During the experiment, the channel was observed on a video monitor (Panasonic WV1410, Japan) and the images recorded (JVC SVHS HR-54900U, Japan) on SVHS videotapes at a rate of thirty frames per second. A digital video mixer (Videonics Digital Video Mixer, Campbell, CA) was used to switch signals between camera assemblies. A title maker (Videonics TitleMaker 3000, Campbell, CA) was used to annotate the videotapes with text. Finally, a digital time stamp (Horita TG-50, Mission Viejo, CA) was used to paste a time on the video images. The timer had a resolution of one video frame or one thirtieth of a second. 


\section{Experiment Recordings}

Once the suspension was placed in the reservoir, the syringe pump began withdrawing it through the channel at a flow rate of $0.18 \mathrm{ml} /$ hour. The experimental flow was videotaped for about one hour. Stopping points on the motorized microscope stage were set approximately 10 $\mathrm{cm}$ from the entrance and about 5 to $8 \mathrm{~cm}$ from the exit of the channel, depending on the experiment. The total distance traveled by the microscope stage was approximately 6 to 8 centimeters. The images contained the entire width of the channel. Refer to figures 3-2 and 3-3 for a schematic representation of the flow, microscope, and recording assemblies.

Recordings were made only after the stage had reached its top speed and as it traveled in the direction opposite to the flow. The stage speed was determined to be $1786 \pm 2$ micrometers per second. The microscope stage platform had been previously machined and markings were placed on it at 0.1 -inch intervals. The stage speed was calculated by measuring the distance with the markings on the stage and the time from the video frames. Similar results were obtained when the speed was measured by observing the markings on a caliper and dividing by the elapsed time. The manufacturer suggested that the variation in the speed was approximately $1 \%$. Assuming a parabolic flow with a wall shear rate of 185 inverse seconds, the stage speed corresponded to a height approximately 13 micrometers from the upper wall of the channel and was approximately equal to the average speed in the channel. While the stage was moving the microscope was focused on labeled particles that appeared to move slowly across the screen and the focus was adjusted so those particles moving slowly from either direction appeared to be in focus. This was accomplished by using a low numerical aperture microscope objective (10x $0.25 \mathrm{NA})$. A side view of the flow and experimental setup is illustrated by the schematic on figure 3-4. 


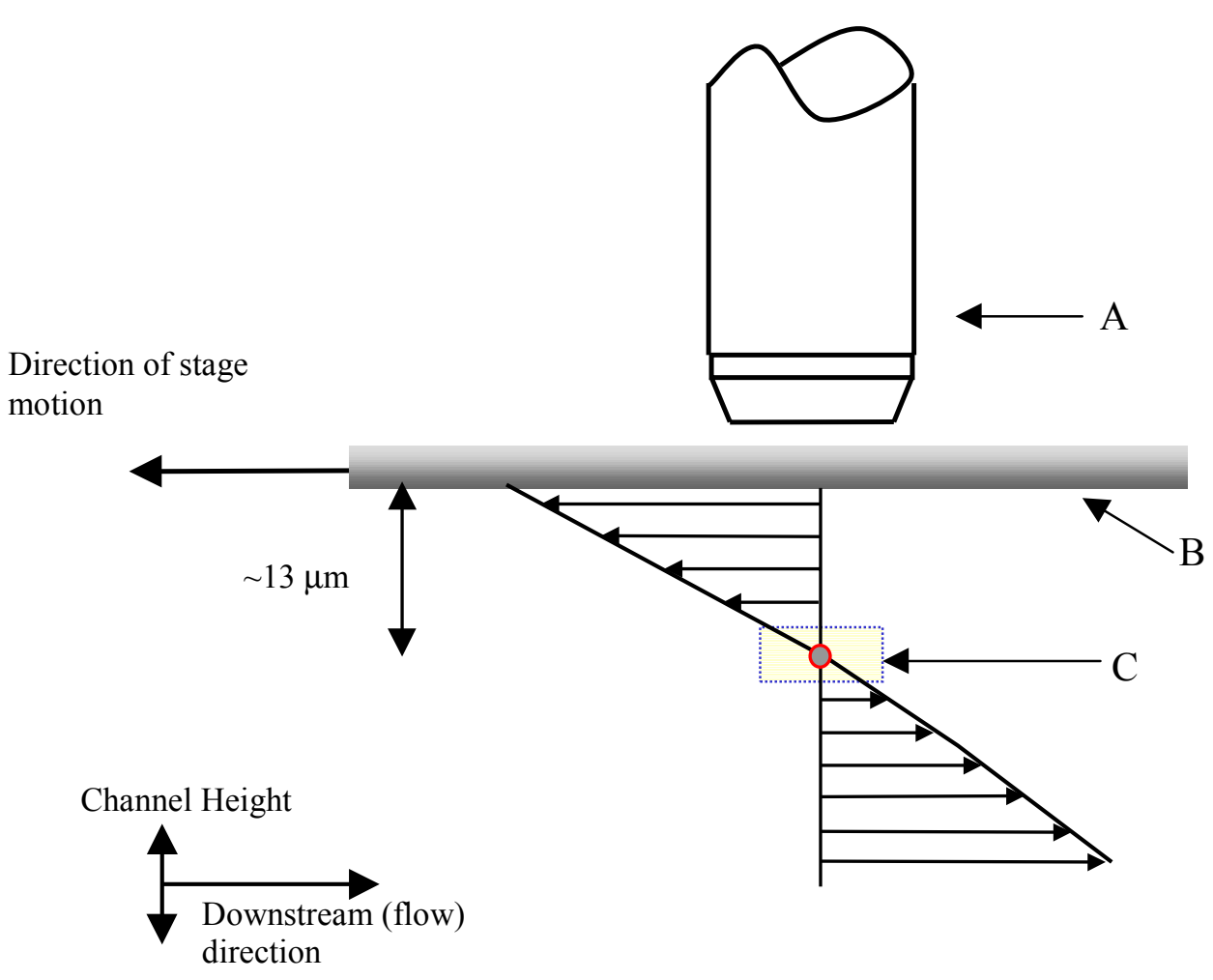

A. Stationary fluorescence objective (10x, $0.25 \mathrm{NA})$ on microscope records top view on videotape.

B. Upper wall of channel mounted on moving motorized microscope stage.

C. Observation zone where labeled cells sometimes appear round and stationary with average velocity profile.

Figure 3-4. Schematic representation of the side view of the experiment.

\section{Data Analysis}

\section{$\underline{\text { Hardware and Software }}$}

The videotapes were processed by replaying them on a SONY medical grade VCR (Sony

SVO 9500MD, Sony Inc., Japan). This VCR allowed manipulation of the tape to display single 
video frames. Its functions included single frame step forward, single frame step reverse, slow forward and slow reverse at 6 frames per second. In the pause mode, the images were not distorted. The video was viewed on a Panasonic monitor (WV-BM 1400, Matsushita Ltd., Japan) and images were imported into a Pentium Pro $200 \mathrm{MHz}$ computer (Dell Computer Corp., Round Rock, TX) with the help of a Matrox frame grabber (Matrox Electronic Systems Ltd, Canada). The video images were then processed with the Metamorph 3.0 package of image-analysis software (Universal Imaging, West Chester, PA).

Data Collection based on Axial Motions in the Moving Reference Frame and Particle Categorization by their initial axial motions

Each video frame was an image of the $\mathrm{x}-\mathrm{z}$ plane, with the flow axis or $\mathrm{x}$-axis in the horizontal direction of the image. The cross-lateral axis, or the channel's width, was the z-axis and it was vertical on the image. The lateral axis, or the channel's height, was the y-axis and it was perpendicular to the image. The entire width of the channel was visible on each image. When the stage was stationary, the flowing particles were moving downstream from left to right. Then, with the stage in motion, particles moved to the left if their speed was slower than that of the stage and to the right if they were faster. Particles traveling at about the same speed as the stage moved slowly in the moving reference frame while particles that appeared stationary were assumed to be moving at the same speed as the stage. The particle motions amounted to a random walk on a line, the $\mathrm{x}$-axis. Since the video images were collected in a regularly intermittent fashion at $30 \mathrm{~Hz}$, the random walk data are a discrete sampling of an apparently continuous process.

Particles were screened for analysis when they met several criteria regarding their axial motion, size, and position in the cross-lateral and axial directions. A cell or a bead became a 
candidate for analysis if it appeared to be relatively stationary. A particle was selected for analysis if it reversed its axial direction in the middle third of the image and its size met a qualitative test based on prior observations of single cells and beads on a slide. Particles were selected if they were approximately 100 micrometers from the wall in the cross-lateral direction. The videotape was reviewed using the slow forward mode (6 frames per second) and small changes in the axial motion of a particle were easily observed.

Figure 3-5 is a four panel schematic that illustrates a first passage measurement. The measurement was started when particles changed their axial direction. At that frame, the particle's position was recorded as its initial axial position $(\mathrm{x}=0)$ in the moving reference frame. This was the last video frame before the particle turned and changed its axial direction. The particles were then categorized based on their initial axial motions. If particles had been moving to the right and then turned to the left, they were called p-type. Similarly, if the particles had been moving to the left and then turned to the right, they were called q-type. The boundaries (or fences) were placed on the digital image and were equally spaced on either side of the particle's centroid. The fence distances were arbitrarily placed at $20,58,117$, and 350 micrometers and they remained fixed on the images until the particular measurement was completed. The videotape was slowly advanced until at least half of the particle had crossed the first boundary on either side (the +20 or -20 -micrometer fence.) The time from the video frame stamp was recorded along with the centroid of the particle. The process was repeated until the labeled bodies crossed one of the fences in each boundary set for the first time. 


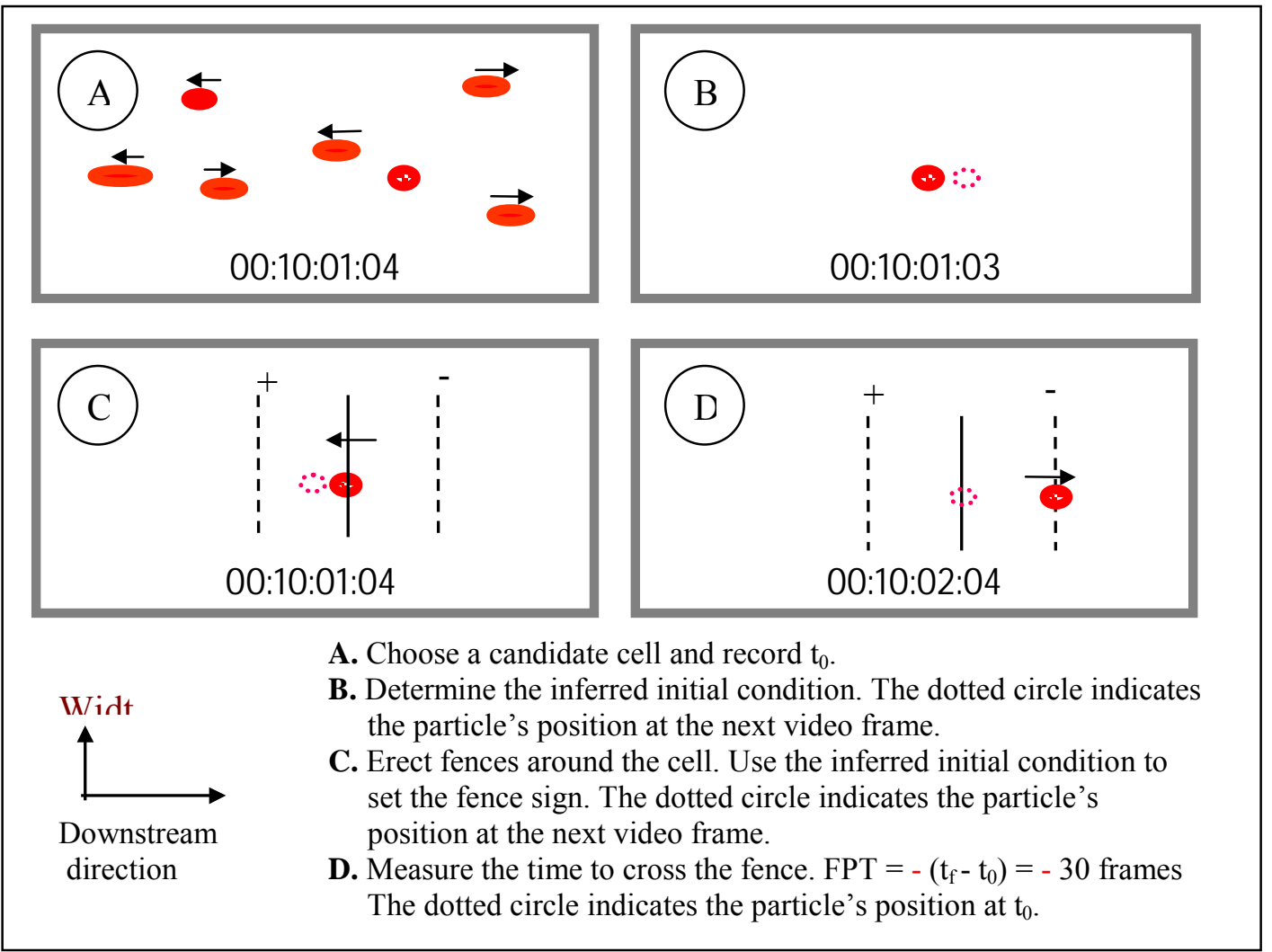

Figure 3-5. Schematic representation of the first passage measurement for a ptype particle.

\section{First Passage Time Calculation}

After the data were collected, the first passage time to reach each fence was calculated using the Microsoft Excel spreadsheet program. The times were first converted to frame counts (30 frames per second). The first passage time was calculated by subtracting the time recorded when the particle was at $\mathrm{x}=0$ from the time recorded when it crossed a fence. The resulting number of frames was then converted back to seconds. 


\section{Grouping of Data}

The table below shows the number of particles that were tracked during the experimental and numerical trials. The type $\mathrm{p}$ and type $\mathrm{q}$ particles were particles with opposite initial axial directions in their motion. This information was obtained over three consecutive video frames. Type $\mathrm{p}$ particles reversed their motion from left to right and they moved from right to left. The opposite was true for type q particles. The numerical simulations used the lateral direction to categorize the simulated particles. Particles that initially moved upward on the lattice, toward the wall, were type p particles because they were moving from right to left in the axial direction. Refer to figure 3-6 for the velocity profiles. The opposite was true for particles that initially moved inward on the lattice, toward the center.

Along with the directional information, each particle's data included the four first passage times required to cross over each of the four selected distances that were placed on either side of the particles initial position. The specific fence position on one side or the other was also noted when the particle crossed the fence for the first time.

Table 3-1. The number of particles tracked during the experimental trials. The type indicates the initial axial direction. Particles with p-type initial direction reversed their motion from left to right and moved from right to left. The opposite was true for q-type particles.

\begin{tabular}{cccccccc}
\hline Trial & & 1 & \multicolumn{2}{c}{2} & & 3 & Total \\
\hline Type & $\mathrm{p}$ & $\mathrm{q}$ & $\mathrm{p}$ & $\mathrm{q}$ & $\mathrm{p}$ & $\mathrm{q}$ & $\mathrm{p} \& \mathrm{q}$ \\
\hline RBC & 128 & 128 & 128 & 128 & 128 & 128 & 768 \\
Beads & 128 & 128 & 128 & 115 & 98 & 98 & 695 \\
\hline
\end{tabular}




\section{Calculated Parameters}

\section{Estimation of the Diffusion Coefficients}

The effective diffusion coefficients were estimated by fitting the data to a theoretical model (14). The coefficient was the estimated slope of the line ( $\left.\mathrm{D}_{\text {eff }}\right)$ that fit Equation 3-1. The subscript (i) indicates the four discrete distances, 20, 58, 117, and 350 micrometers. The constant $\mathrm{k}$ lumped together parameters that took into account the channel's half height, $\mathrm{R}$; the maximum velocity, $\mathrm{U}_{\max }$; and the particle's initial lateral position, $\mathrm{r}_{\mathrm{o}}$. The maximum velocity was estimated to be $0.25 \mathrm{~cm} / \mathrm{sec}$, the channel half height was measured to be $0.0027 \mathrm{~cm}$, and the initial lateral position was estimated to be $0.0014 \mathrm{~cm}$.

$$
\mathrm{x}_{\mathrm{i}}^{2}=\mathrm{D}_{\text {eff }} \boldsymbol{C}<\mathrm{t}>_{\mathrm{i}}+\mathrm{k}<\mathrm{t}>_{\mathrm{i}}^{3} \boldsymbol{h}
$$

\section{Equation 3-1}

where, $\mathrm{k}=\frac{8}{3} \mathrm{a}^{2} \mathrm{r}_{\mathrm{o}}^{2} ; \mathrm{a}=\frac{\mathrm{U}_{\max }}{\mathrm{R}}$

\section{$\underline{\text { Calculations of Conditional Probability Fractions }}$}

The first passage times were assigned labels in two different ways to indicate which of the two lines or fences at the discrete distances was crossed by the particle. These conventions are explained further below. The sign labels were arbitrarily chosen for their ease of use with the spreadsheets because time numbers with such a label were still considered numbers (rather than text) by the spreadsheet and thus they were easily manipulated. The fractions of particles crossing a given fence from each pair of fences were then calculated. This fraction was the proportion of the $(+)$ labeled FPTs out of the total number for each experimental trial at each fence distance. 
In the first way, when particles crossed a fence to the right of their initial position, the FPT was labeled with a $(+)$ sign. If the particles crossed a fence to the left of their initial position the FPT was labeled with a (-) sign. The fractions were calculated in a second way using the particle's initial direction to label the FPT. If a particle crossed a fence in the same direction as its initial direction the FPT was labeled with a $(+)$ sign. If a particle crossed a fence in the opposite direction the FPT was labeled with a (-) sign. In the first calculation, only the initial position was considered and thus the model followed the concepts associated with a first order Markov process. Two pieces of information were used in the second calculation, a position and a direction of motion, consistent with concepts tied to a second order Markov process. For either way, the formula was:

Conditional Probability Fraction $=\frac{\# \text { particles with a "positive"FPT }}{\text { All particles }}$ Equation 3-2

\section{Estimation of the character of diffusion}

Following Compte's treatment (10), a number to describe the nature of diffusion that characterized the lateral motions was obtained by fitting the data to a theoretical model that related the axial squared displacement (the square of the fence distances) to the average first passage time to reach each fence. The data, average FPT vs. the squared displacement, were fitted using a power law fit and the exponent, the parameter alpha in equation 3-3, was compared to theoretical values. The subscript was the same as in equation 3-1. Theoretically, the character of the lateral motions is considered diffusive for values of alpha equal to three, superdiffusive for values less than three, and subdiffusive for values higher than three.

$$
\mathrm{x}_{\mathrm{i}}^{2} \propto<\mathrm{t}>_{\mathrm{i}}^{\alpha}, \quad \text { Equation 3-3 }
$$




\section{Computer Simulations}

\section{Overview of the Simulations}

Programs to simulate particle motions in the axial and lateral directions were written in the $\mathrm{C}++$ computer language. The experimental observations showed no appreciable motions in the cross-lateral direction and thus the model was limited to two dimensions. Particles were modeled as points and their lateral motions were simulated by two different methods. The focus of this effort was to reproduce the basic events of the mechanism for lateral dispersion within a probabilistic framework; it was not to replicate with accuracy specific flow parameters or physical characteristics of the events in the suspension.

In all the simulations, the axial motions were modeled as convective. The Poiseuille formula was used to determine the velocity as a function of the particle's lateral position. Refer to figure 3-6 for a schematic representation of the velocity profile. All particles were started from the same lateral position, which corresponded to the position that would give a speed equal to the speed of the microscope stage. Equal numbers of particles were started in each lateral direction. The lateral motions were modeled as random walks on a lattice and particles changed their position at fixed time intervals. The points in the lattice were equidistant. The word "random" is taken to imply a more general process than the very specific Gaussian one, which is connected to the normal distribution.

In one type of simulation, lateral motions were modeled by using a binomial process to decide the lateral direction of a single step after each time interval. This amounted to a simple random walk (SRW) on a line. In the limit for many steps with this case, the distribution of particles on the line is statistically described by the Gaussian distribution. In the second type of 


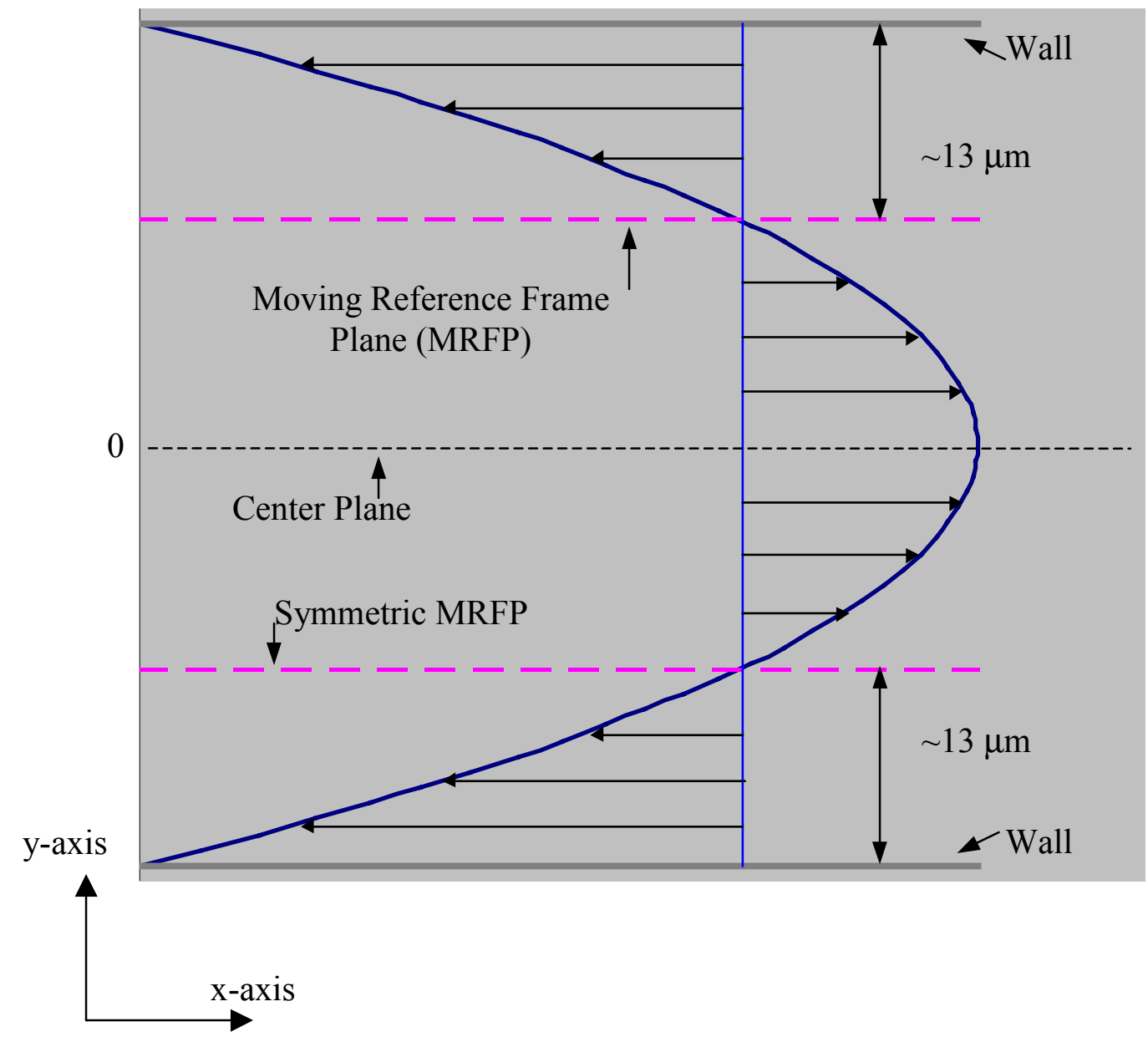

Figure 3-6. Schematic of the flow profiles in the moving reference frame. The parabolic line indicates the flow profile in the channel frame. Particles located on the moving reference frame planes had zero velocity.

simulation, the steps, or lack of a step, in a given direction were determined by a Poisson distributed integer variable. After all the steps in one direction were completed, the sign of the lateral direction was reversed and the particle moved in the new direction for a new number of steps and so on. This type of random walk was designated as a Kac walk (KW) and had a "persistence" associated with its lateral motion (24).

All axial motions were transformed to a moving reference frame by offsetting the particle's speed by a constant speed, which corresponded to the speed of the microscope stage 
during the experimental trials. The particle's speed was a function of its lateral position. For all simulations, after each lateral motion, the particles were translated axially by a distance that was the product of the particle's moving reference frame velocity and the time step. The program was written so that the particles would be reflected from a lateral boundary (wall), but that never occurred in these simulations. The size of the lattice (the lateral step) and the time step were manipulated so result outcomes similar to the experimental RBC data were obtained. As with the experiments, first passage time data and particle positions were recorded from each numerical trial. The number of data generated by the simulations is listed on Table 3-2.

Table 3-2. The number of particles "tracked" with the numerical trials. The type indicates the initial lateral direction. P-type particles had an initial lateral direction toward the "wall" and q-type particles had an initial lateral direction toward the "centerline."

\begin{tabular}{cccccccc}
\hline Trial & & 1 & \multicolumn{2}{c}{2} & & 3 & Total \\
\hline Type & $\mathrm{p}$ & $\mathrm{q}$ & $\mathrm{p}$ & $\mathrm{q}$ & $\mathrm{p}$ & $\mathrm{q}$ & $\mathrm{p} \& \mathrm{q}$ \\
\hline SRW & 850 & 850 & 850 & 850 & 850 & 850 & 5100 \\
KW & 850 & 850 & 850 & 850 & 850 & 850 & 5100 \\
\hline
\end{tabular}

\section{Random Number Generation}

The $\mathrm{C}++$ code was written and compiled using Microsoft Visual Studio $97^{\circledR}$ and it appears in the appendix. The compiler's uniform random number generator, which produced values between 0 and 1, was used to pick the direction of each lateral step in the simple random walk simulation. Values above 0.5 always resulted in a step in one direction (toward the wall), while those below 0.5 resulted in a step to the opposite direction. The Poisson random numbers were generated with the uniform random number generator and an inversion method $(13 ; 16)$. A large number of Poisson distributed numbers were created using the Random number generator 
in Microsoft Excel $97^{\circledR}$. The cumulative distribution function of these variables was then plotted. Each variable in the distribution was a fraction in the range from zero to one. For example, from a Poisson distribution with mean and variance of four, the number zero could be generated when the uniform number generator returned a value between 0 and 0.01833 . Similarly, number one could be generated when the random number generated was between 0.018331 and 0.0911 , and so on. The code for the generation of several Poisson distributions is included in the appendix.

\section{Flowcharts of the Numerical Algorithms}

The flowchart for the program that models the lateral motions of particles using the simple random walk is in figure 3-7. The flowchart for the Kac walk is in figure 3-8. Both simulations were done in a moving reference frame, as were the experiments. 
Figure 3-7. Flowchart of the simple random walk simulation for one particle. The algorithm was repeated for many particles.

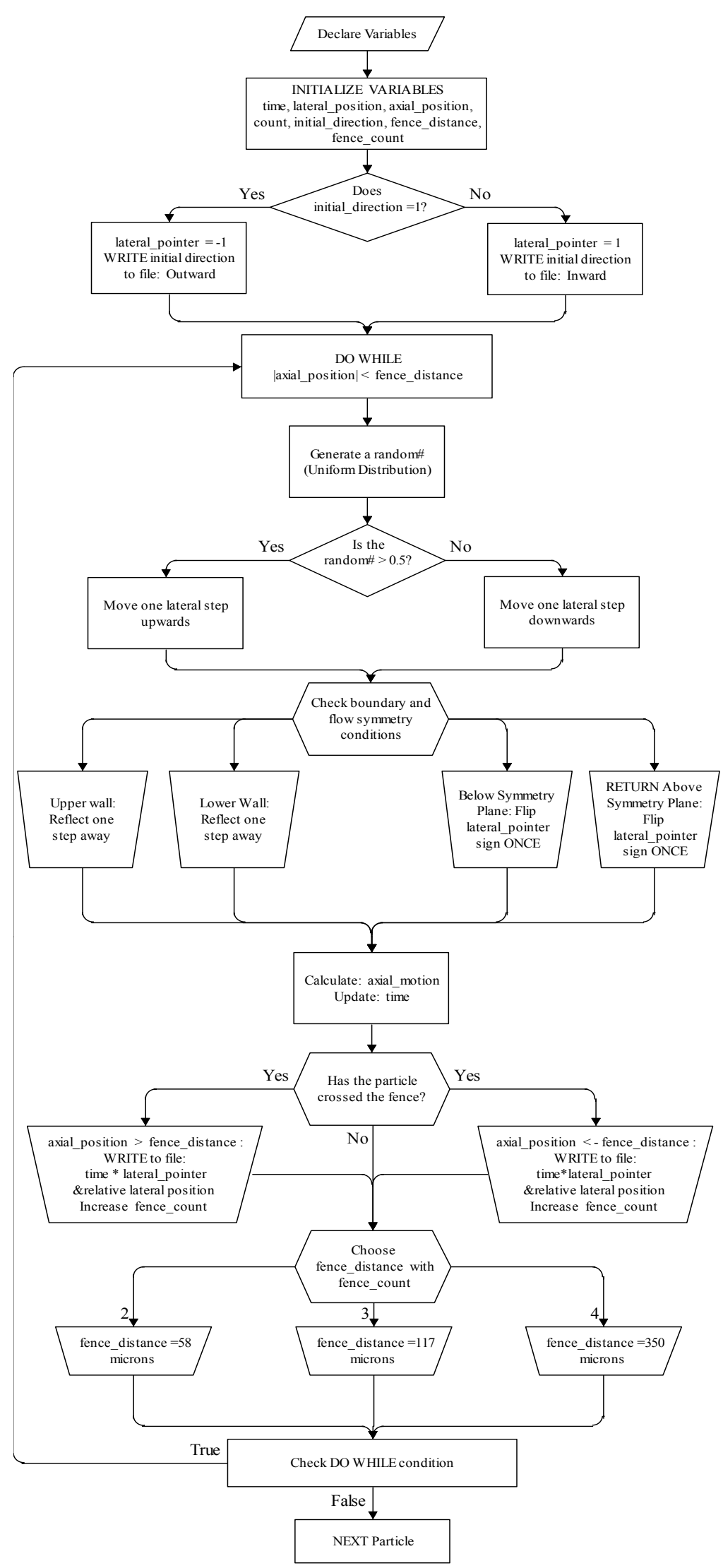


Figure 3-8. Flowchart of the Kac walk simulation for one particle. The algorithm was repeated for many particles.

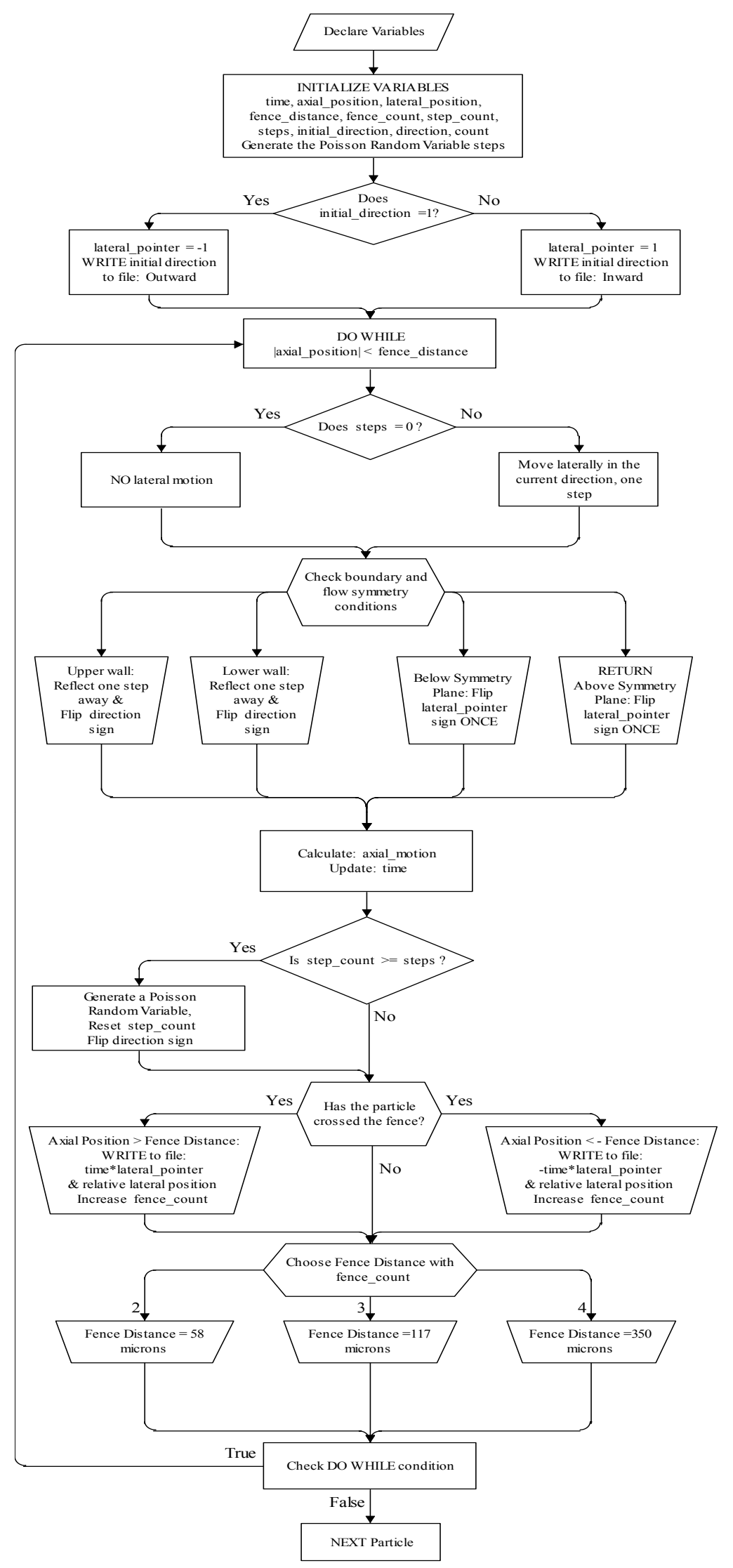




\section{CHAPTER 4. RESULTS}

The random motions of red blood cells and beads were characterized. Three trials with human red blood cells were conducted in flowing suspensions with concentration of $25 \%$ by volume. Three trials with 0.46 -micrometer diameter beads were also conducted in flowing suspensions with concentration of $0.003 \%$ by volume and all particles were tracked in a moving reference frame. Data from 256 cells were collected from recordings of each red cell trial and data from 256, 243, and 196 beads were collected from recordings of the dilute bead trials. The suspensions were withdrawn through thin glass channels ( $54 \times 556$ micrometers) with a flow rate of 0.180 milliliters per hour. The wall shear rate was estimated to be approximately $185 \mathrm{sec}^{-1}$, assuming Poiseuille flow, and the local shear rate at the moving reference frame was estimated to be approximately $100 \sec ^{-1}$ ( 14 micrometers from the center plane of the thin channel).

An initial direction of motion and four (first passage) times to reach each of four distances were recorded for each particle. The average times for each distance were calculated and used to estimate the effective dispersion coefficient and the nature of the random motions.

The random motions of particles were also simulated numerically in two dimensions. The axial motions were modeled as convective and the lateral motions were simulated as stochastic. The lateral motions were modeled using a simple random walk and again using a Kac walk. Three trials with 1700 particles were simulated for each type of random walk. Motions were simulated in a moving reference frame and the data were collected and analyzed as in the experimental trials. 


\section{Summary of Results}

The effective diffusion coefficient of the 0.46 -micrometer diameter beads was estimated to be $(3.75 \pm 0.59) \times 10^{-9} \mathrm{~cm}^{2} / \mathrm{sec}$ and that of red cells $(8.81 \pm 1.73) \times 10^{-9} \mathrm{~cm}^{2} / \mathrm{sec}$. The erratic behavior of the beads followed the exponent for ideal Brownian motion while that for the red cells matched values for anomalous diffusion. More than one half of the particles in all experimental trials crossed a fence in the same direction as in their initial direction of motion and while the fractions decreased with distance, they always remained above one half.

The effective diffusion coefficients predicted by the numerical results were in the order

of $\sim 7 \times 10^{-9} \mathrm{~cm}^{2} / \mathrm{sec}$ for both the simple random walk and the Kac walk. The simple random walk simulations, which followed ideal Brownian motion, failed to predict the fractions of particles crossing a fence in the direction of their initial motion, but did match the time distributions and the fractions of particles crossing the downstream fence. The simple random walk simulation resulted in approximately equal number of particles crossing in either direction relative to their initial direction of motion. The Kac walk simulations matched all the observations from the RBC trials.

\section{General Observations and Descriptive Graphs of First Passage Time Data}

The RBC appeared to change their directions of motion more often than the beads; they appeared to have a more erratic pattern to their motion. On the average, the RBC traveled their net distances faster than the beads.

The combined average first passage time at each distance for all RBC and bead trials are shown in figure 4-1, and the results for the average first passage times of the SRW and KW 


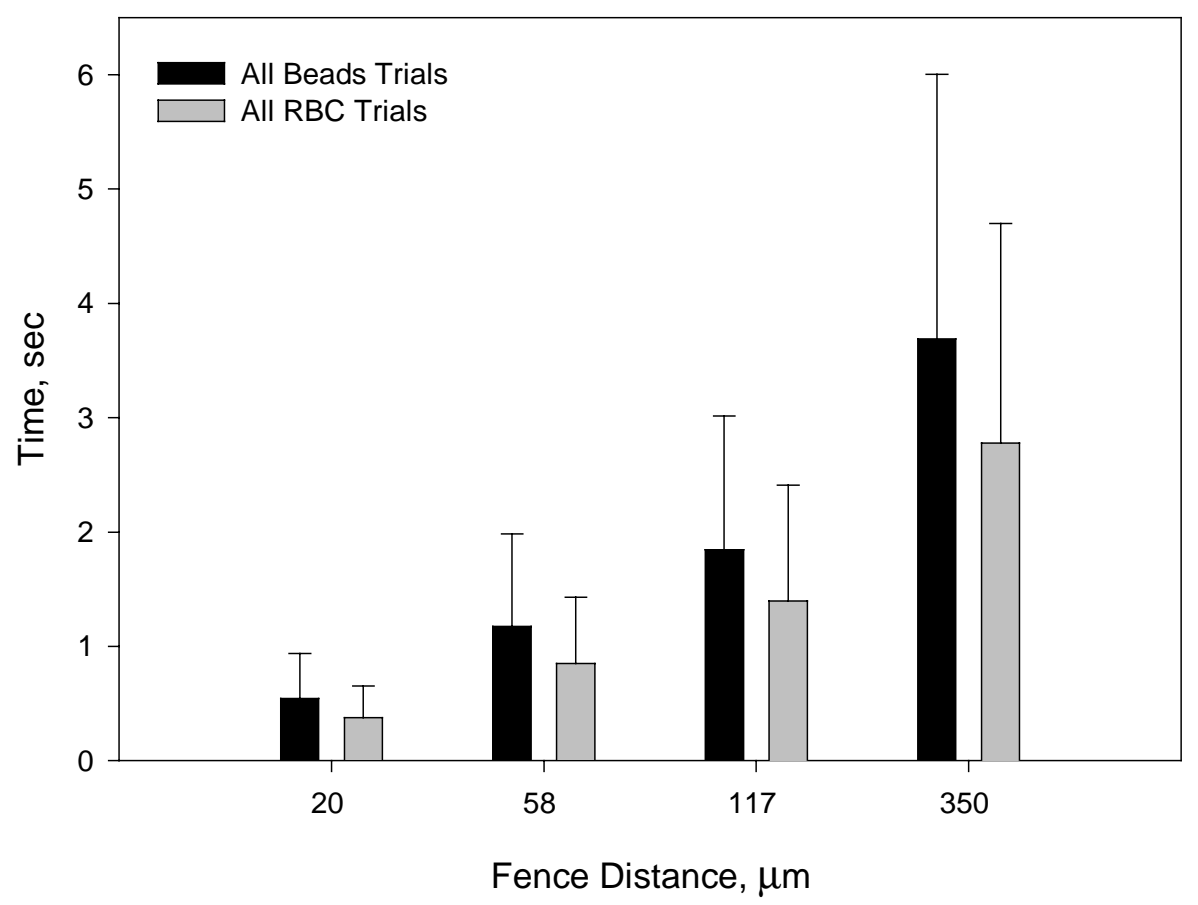

Figure 4-1. Average first passage time \pm standard deviation for combined experimental trials.

simulations are shown in figure 4-2. As it can be seen in these figures, the numerically simulated first passage times, for both the SRW and KW, matched the RBC values well. The bar charts show the average first passage time at each distance with an error bar that represents the standard deviation. The average first passage times from all the experimental trials are listed in table 4-1 and those from the numerical simulations in table 4-2.

The cumulative probability distribution graphs for the first passage times from the Beads and RBC trials are shown in figures 4-3 and 4-4. These graphs show the probability that a particle would have crossed a fence after a certain time. The figures show that most of the particles reach the short fences quickly, but that number decays with distance as can be seen by the decreasing slope of the cumulative probability lines with fence distance. Figure 4-5 shows 


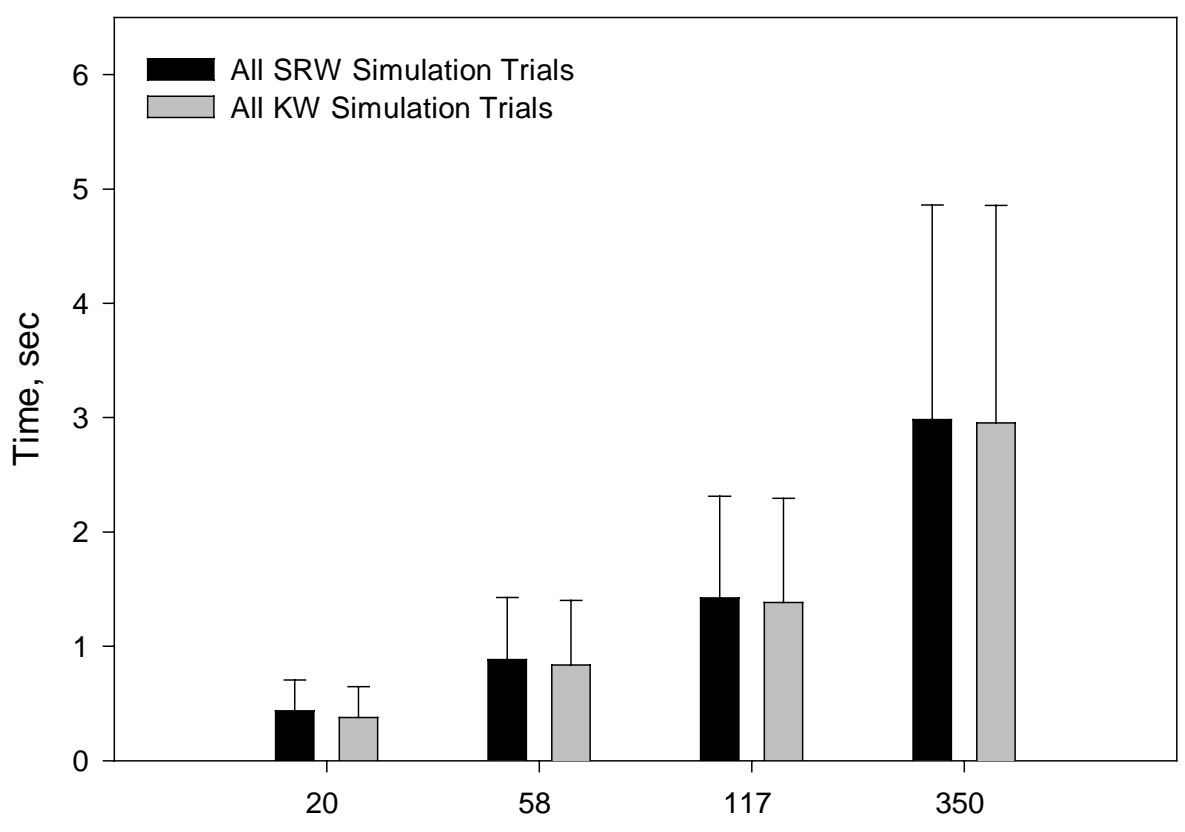

Fence Distance, $\mu \mathrm{m}$

Figure 4-2. Average first passage time \pm standard deviation for combined numerical trials.

Table 4-1. Experimental results - Average first passage times for each trial.

\begin{tabular}{cccccccc}
\hline \multicolumn{7}{c}{ Average First Passage Times, seconds } \\
\hline Experiment & \multicolumn{3}{c}{ Beads } & \multicolumn{3}{c}{ Red Blood Cells } \\
\hline Distance, $\mu \mathrm{m}$ & Trial & 1 & 2 & 3 & 1 & 2 & 3 \\
\hline 20 & 0.573 & 0.526 & 0.526 & 0.357 & 0.374 & 0.393 \\
58 & 1.184 & 1.191 & 1.191 & 0.802 & 0.862 & 0.881 \\
117 & 1.895 & 1.791 & 1.791 & 1.323 & 1.404 & 1.459 \\
350 & 3.756 & 3.489 & 3.489 & 2.695 & 2.618 & 3.020 \\
\hline
\end{tabular}

Table 4-2. Numerical results - Average first passage times for each trial.

\begin{tabular}{|c|c|c|c|c|c|c|c|}
\hline \multicolumn{8}{|c|}{ Average First Passage Times, seconds } \\
\hline \multicolumn{2}{|c|}{ Simulation } & \multicolumn{3}{|c|}{ Simple Random Walk } & \multicolumn{3}{|c|}{ Kac Walk } \\
\hline Distance, $\mu \mathrm{m}$ & Trial & 1 & 2 & 3 & 1 & 2 & 3 \\
\hline 20 & & 0.442 & 0.434 & 0.434 & 0.379 & 0.375 & 0.382 \\
\hline 58 & & 0.886 & 0.883 & 0.880 & 0.841 & 0.838 & 0.832 \\
\hline 117 & & 1.420 & 1.424 & 1.426 & 1.378 & 1.387 & 1.385 \\
\hline 350 & & 3.001 & 2.922 & 3.030 & 2.965 & 2.965 & 2.938 \\
\hline
\end{tabular}




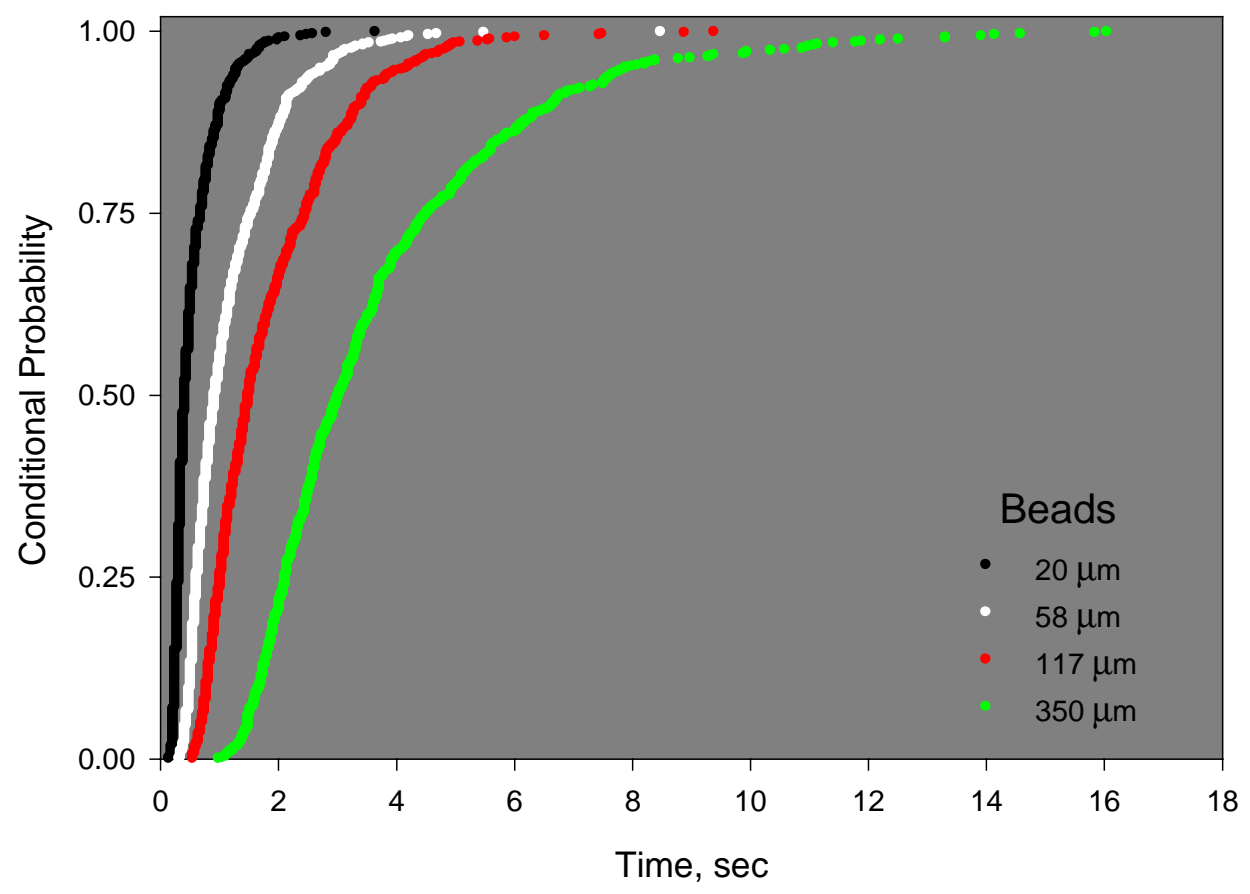

Figure 4-3. Experimental result - Cumulative probability distributions for the first passage times of the bead trials.

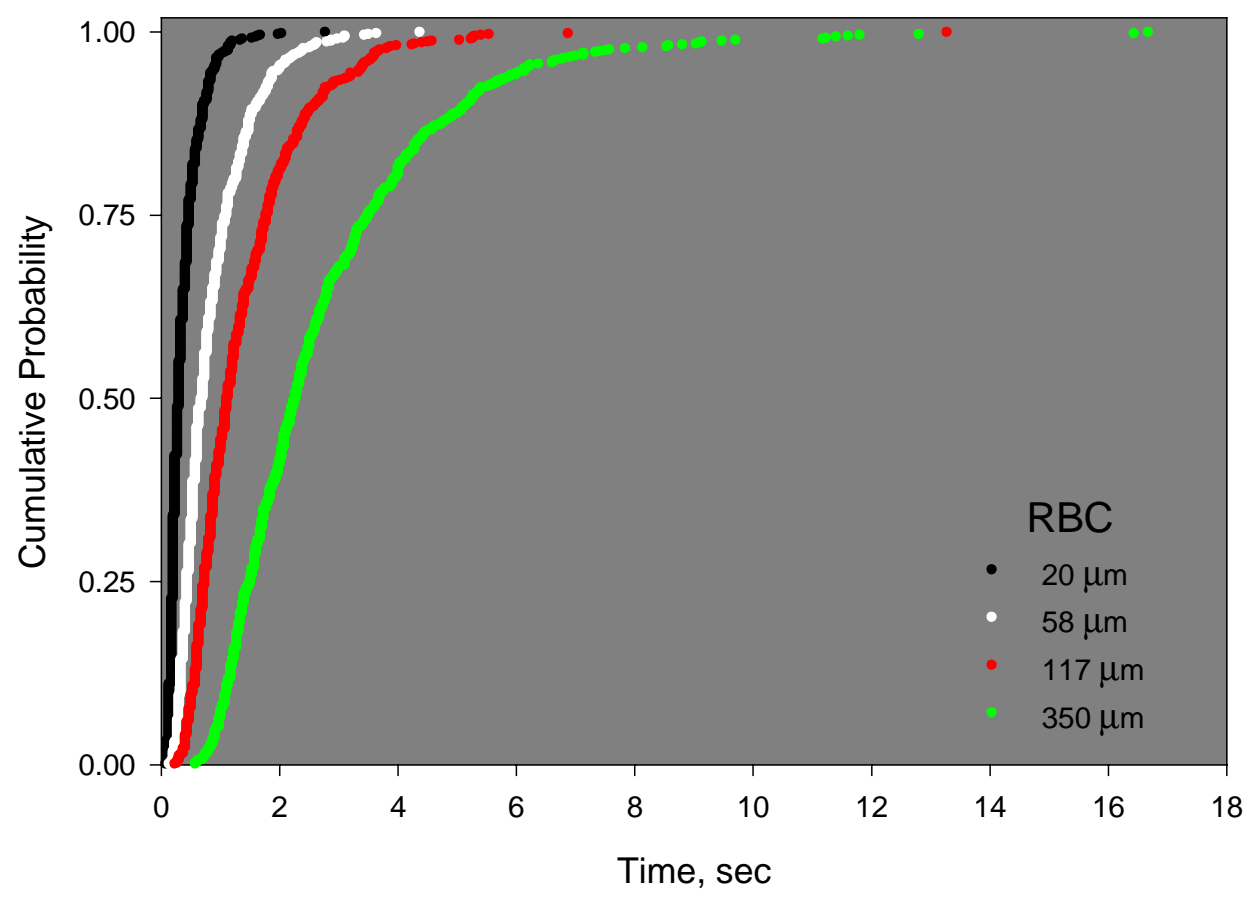

Figure 4-4. Experimental results - Cumulative probability distributions for the first passage times of the RBC trials. 


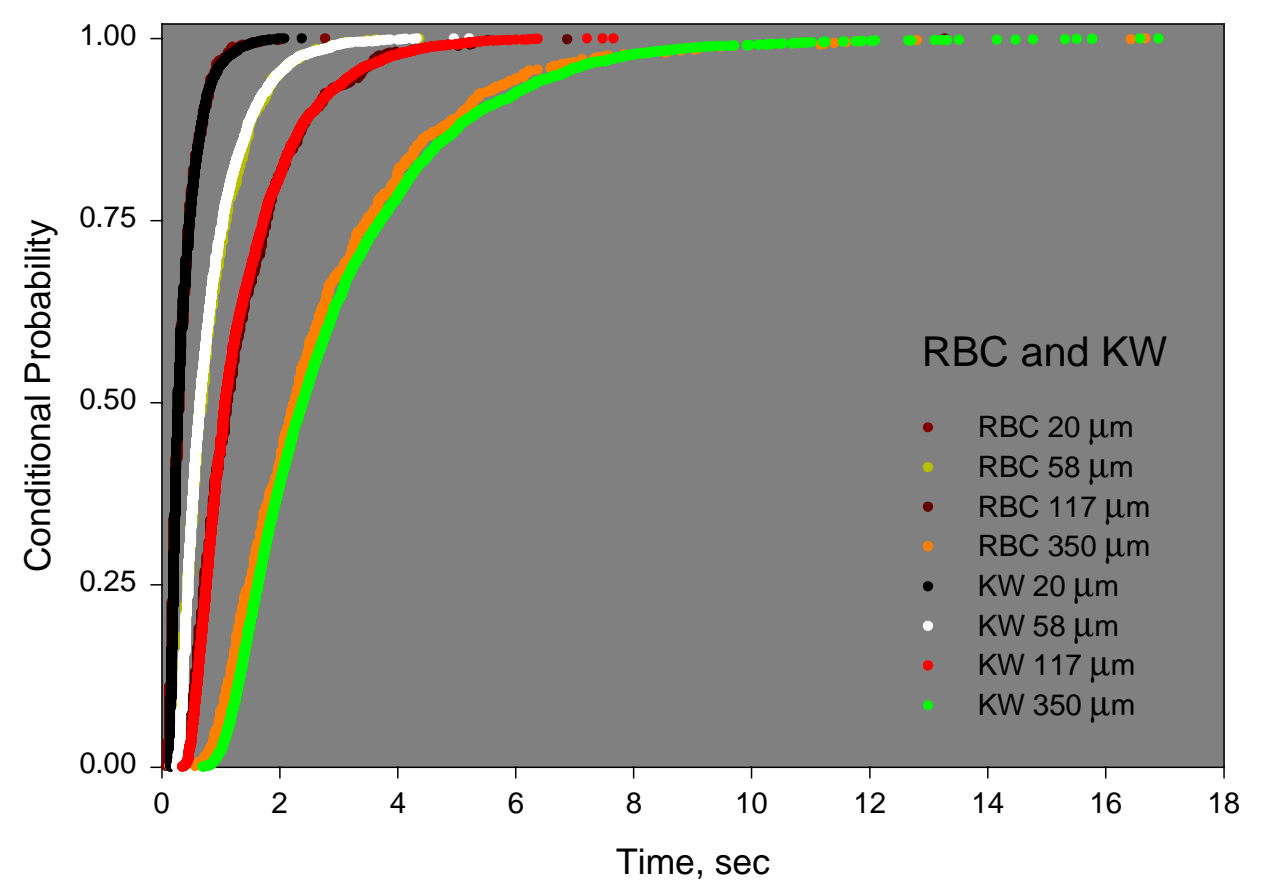

Figure 4-5. Cumulative probability distributions of the first passage times for combined KW numerical trials and RBC experimental trials.

the good agreement obtained between the experimental RBC trials and the KW simulation. The agreement in the cumulative distributions of the $\mathrm{RBC}$ and $\mathrm{KW}$ is not as good at the 350 micrometer fence and that is probably due to slight blunting of the velocity profile in the experimental trials. Similar data were obtained with the SRW simulation. Interestingly, the average [first passage] is a powerful scaling parameter. Figure 4-6 shows the scaled cumulative probability graphs of all 48 sets of experimental and simulated data sets (12 trials each with 4 fence distances). In that graph, each time point was scaled by the average time for that fence and trial.

The experimental results for the fractions of particles crossing a fence in the same axial direction as their initial direction of motion are shown in figure 4-7. Large fractions of both the $\mathrm{RBC}$ and the beads crossed a fence in the direction of their initial motion and these results are 


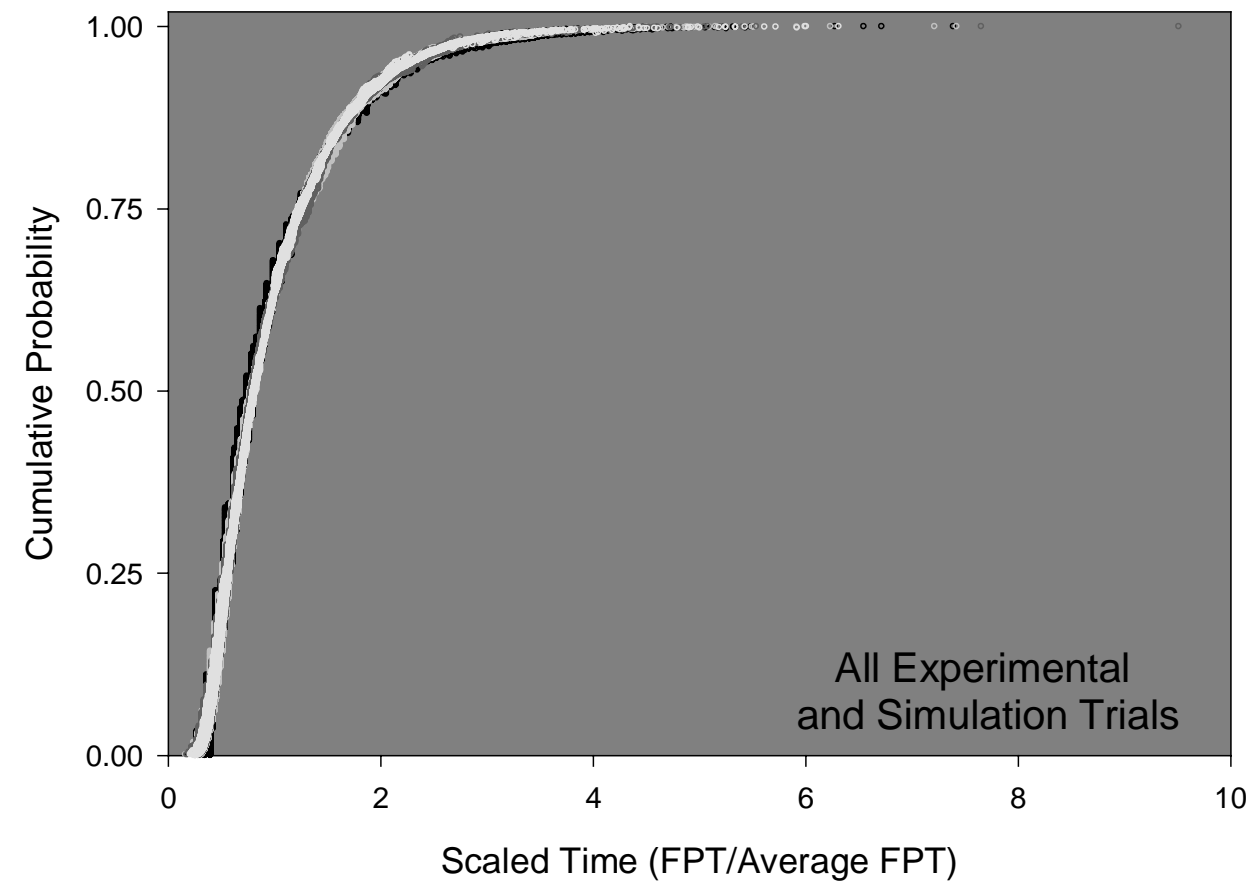

Figure 4-6. Scaled cumulative probability distributions for all experimental and simulated trials at each fence distance. The first passage times were scaled by the mean first passage time at each fence distance.

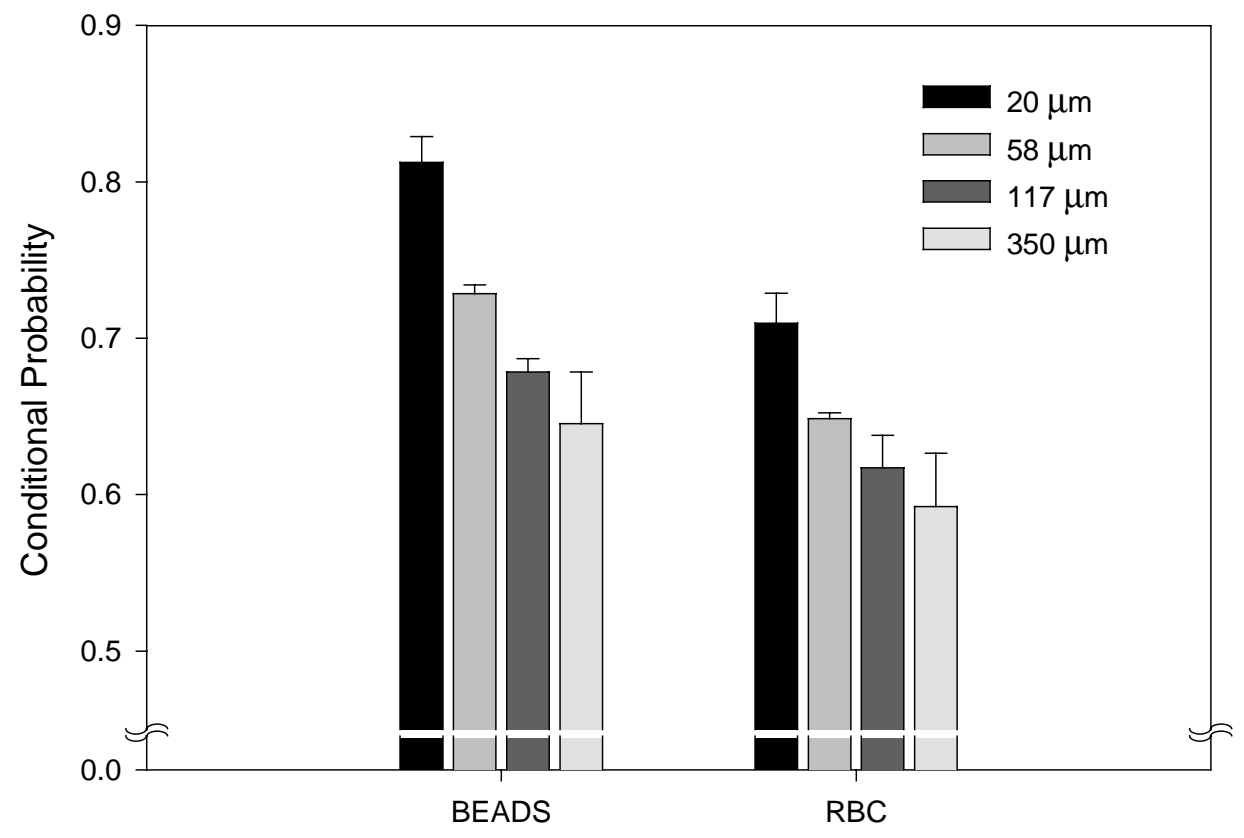

Figure 4-7. Experimental results - Fractions of particles crossing a fence in the same direction as their initial direction of motion. 
consistent with the second order Markov process. Figure 4-8 shows that there was no difference between the p-type and q-type particles and presumably that is an indication that the particles were exposed to a region with linear shear rate. Figure 4-9 shows the experimental fractions of particles crossing a fence in the downstream direction. Both the beads and the $\mathrm{RBC}$ fractions are approximately $50 \%$ as would be expected when the first order Markov model is used to calculate these values. The equal number of cells crossing the downstream fence also indicates that over the time that these particles were studied there was no significant amount of settling. Figure 4-10 shows that there was no significant difference in the RBC and $\mathrm{KW}$ results for the fractions of particles crossing a fence in the direction of their initial motion. In addition, all simulations gave fractions around $50 \%$ for the number of particles crossing the downstream fences.

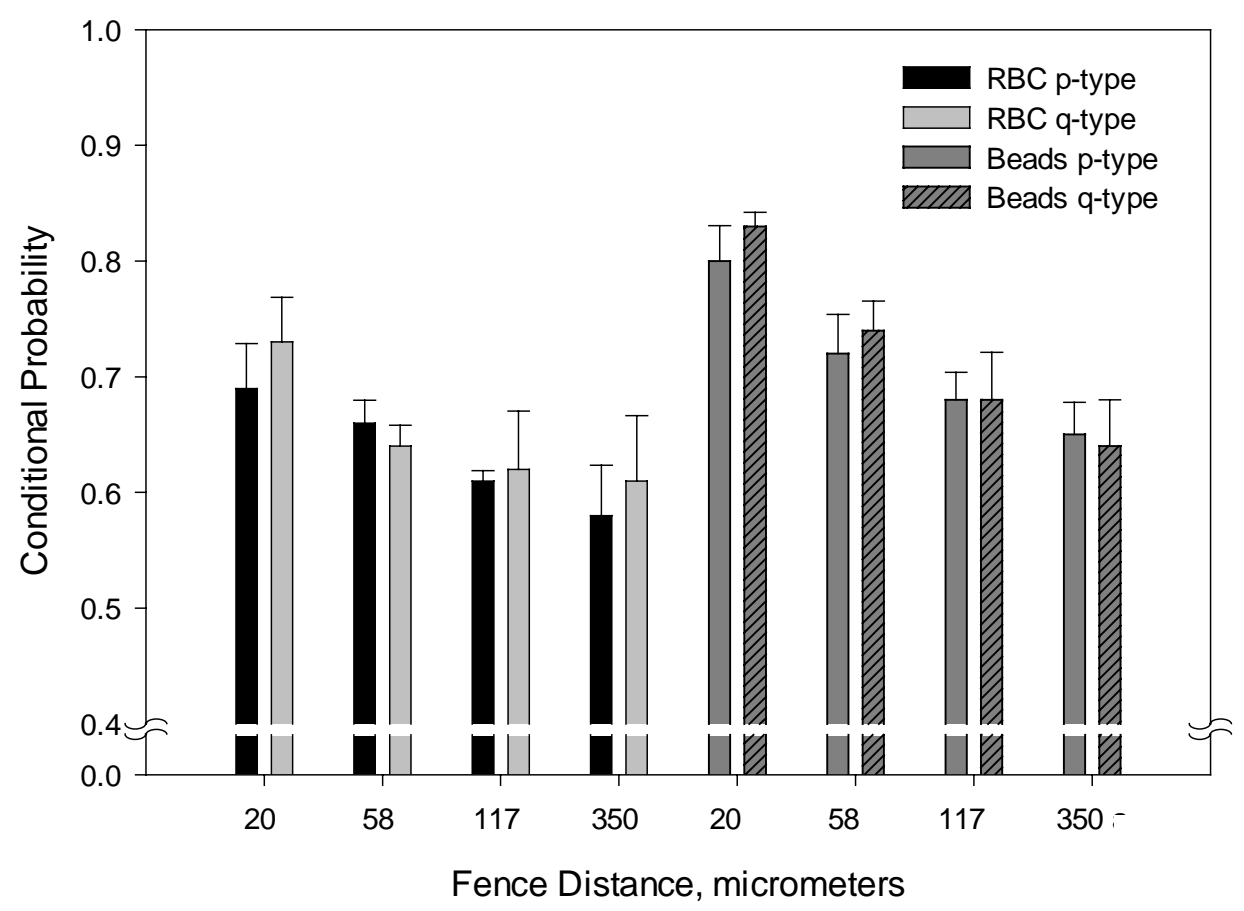

Figure 4-8. Experimental results - Fractions of p- and q-type particles crossing a fence in the direction of their initial motion. There was no significant difference between $\mathrm{p}$ - and q-type particles 


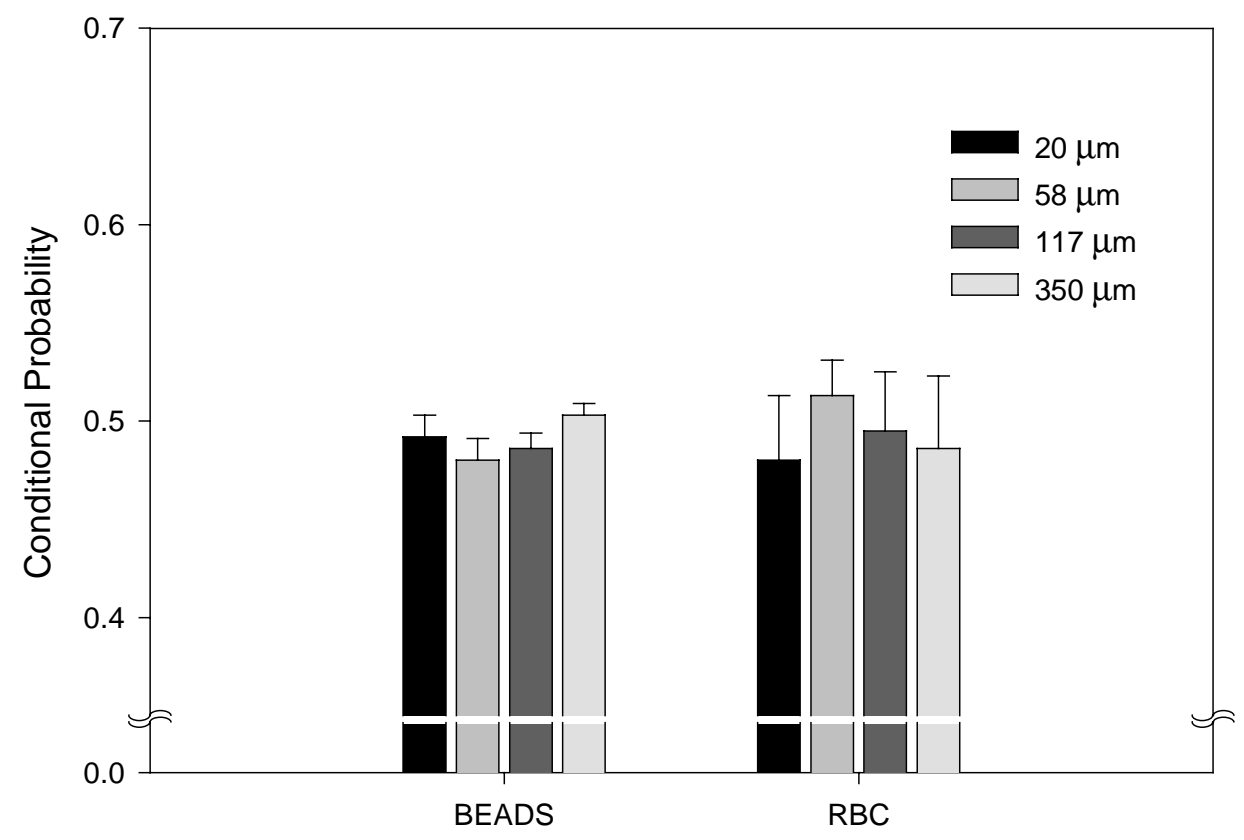

Figure 4-9. Experimental results - Fractions of particles crossing a fence in the downstream direction

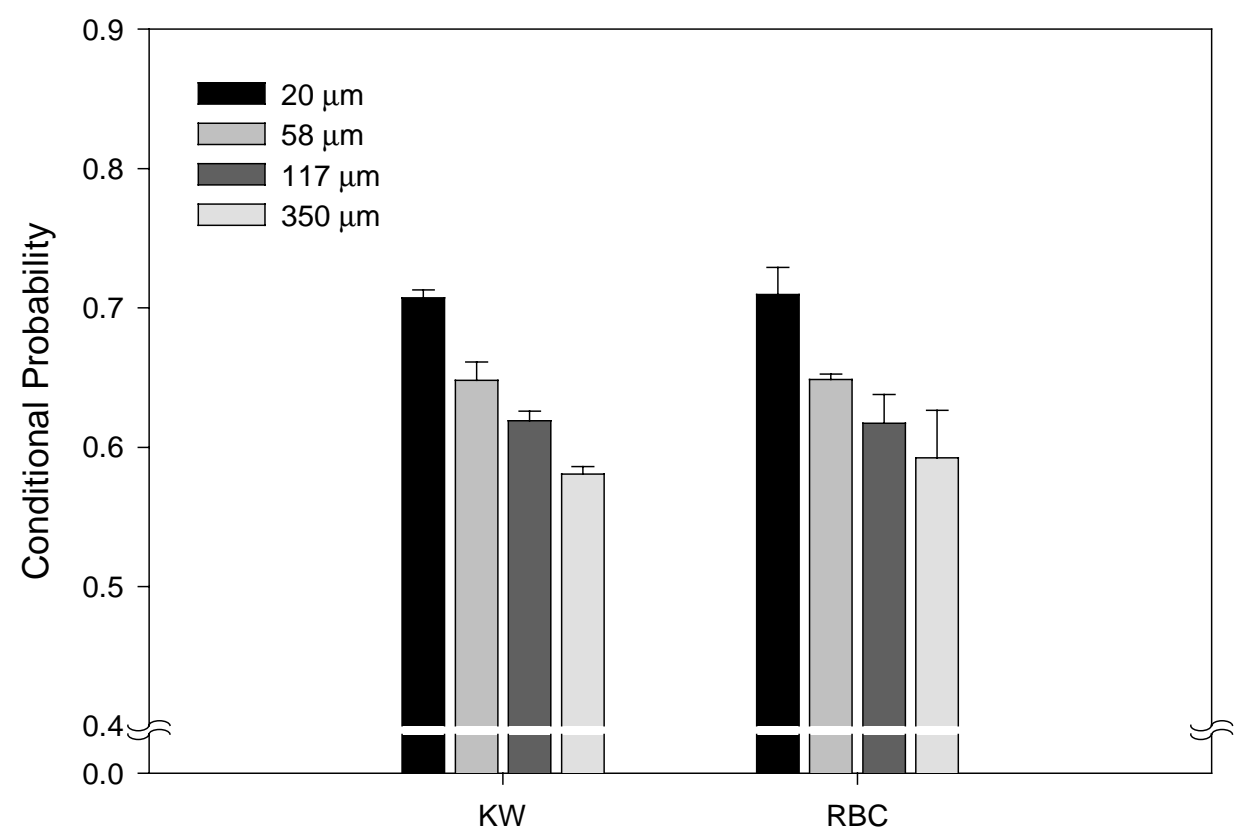

Figure 4-10. Comparison of numerical simulation (KW) results to the experimental trials $(\mathrm{RBC})$. 
The SRW simulation also produced fractions around $50 \%$ for the cells crossing a fence in the direction of their initial motion and that result was expected since the SRW model is a first order Markov Process.

\section{Estimation of the Effective Diffusion Coefficients}

The effective diffusion coefficient, $\mathrm{D}_{\text {eff }}$, for beads and red cells was estimated by the slope of the linear regression line on the plot of the scaled average first passage time vs. the squared axial displacement $\left(\mathrm{x}^{2}=\mathrm{D}_{\text {eff }}\left(2<\mathrm{t}>+\mathrm{k}<\mathrm{t}>^{3}\right)\right.$. The average value for the beads was approximately $4 \times 10^{-9} \mathrm{~cm}^{2} / \mathrm{sec}$ and for the red cells $0.9 \times 10^{-9} \mathrm{~cm}^{2} / \mathrm{sec}$. Figure $4-11$ shows the plots of the experimental data and table 4-3 shows the values for the effective diffusion coefficient for each experimental trial. Table 4-4 shows the effective dispersion coefficients from each numerical trial and all results would cluster around the RBC 3 line in figure 4-11.

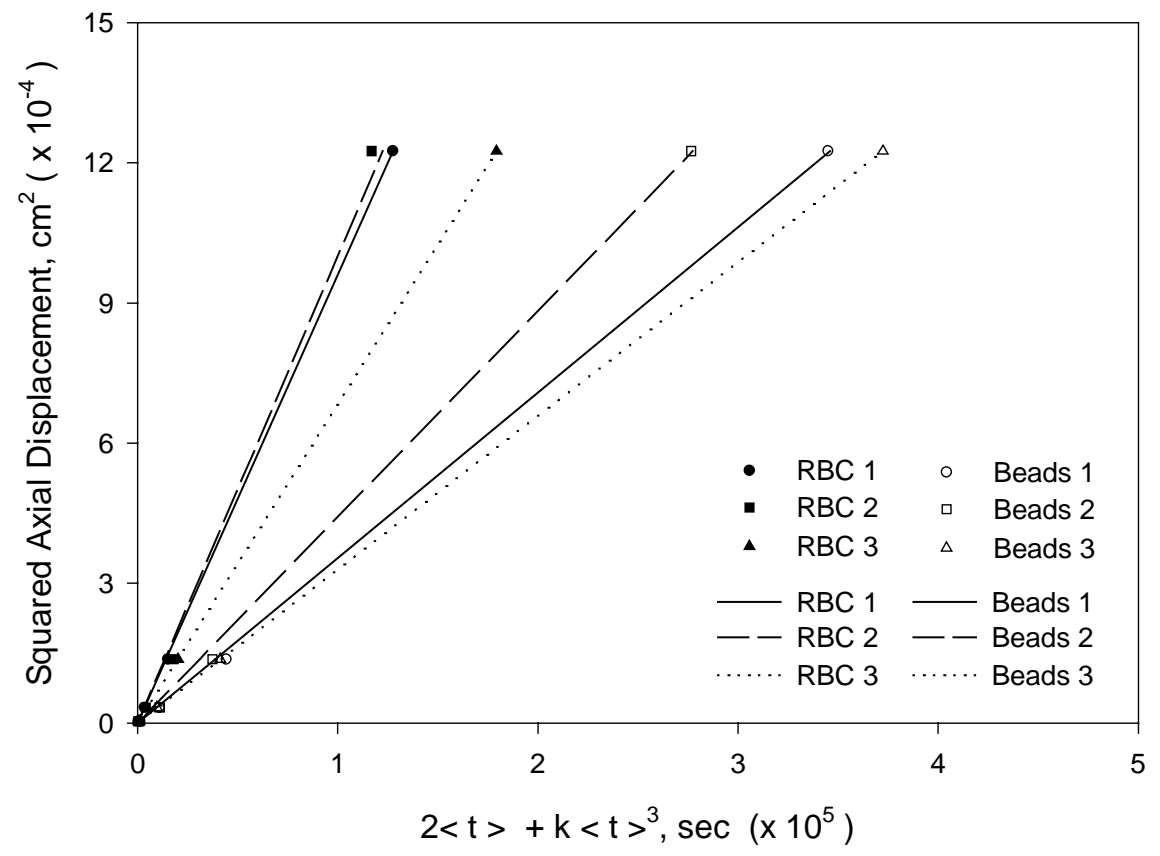

Figure 4-11. Linear regression fits on the plot of the scaled average FPT vs. the squared axial displacement. The symbols represent the data and the lines represent the regression fits. 
Table 4-3. Experimental results - Effective diffusion coefficients. The values were the slopes of the linear regression lines on the plots of the scaled average FPT vs. the squared displacement (figure 4-11).

\begin{tabular}{|c|c|c|c|c|c|c|}
\hline \multicolumn{7}{|c|}{$\begin{array}{l}\text { Experimental Estimates of Effective Diffusion Coefficients }\left[\mathrm{cm}^{2} / \mathrm{sec}\right] \\
\qquad \mathbf{x}^{2}=D_{\text {eff }}\left(2<t>+k<t>^{3}\right)\end{array}$} \\
\hline \multirow{2}{*}{$\begin{array}{l}\text { Experiment } \\
\text { Trial \# }\end{array}$} & \multicolumn{3}{|c|}{ Beads } & \multicolumn{3}{|c|}{ RBC } \\
\hline & 1 & 2 & 3 & 1 & 2 & 3 \\
\hline $\mathbf{D}_{\text {eff }}$ & $3.54 \times 10^{-9}$ & $4.41 \times 10^{-9}$ & $3.29 \times 10^{-9}$ & $9.60 \times 10^{-9}$ & $1.00 \times 10^{-8}$ & $6.83 \times 10^{-9}$ \\
\hline $\mathbf{r}^{2}$ & 0.999 & 0.999 & 0.999 & 0.999 & 0.997 & 0.999 \\
\hline Average & \multicolumn{3}{|c|}{$3.75 \times 10^{-9}$} & \multicolumn{3}{|c|}{$8.81 \times 10^{-9}$} \\
\hline S.D. & \multicolumn{3}{|c|}{$0.59 \times 10^{-9}$} & \multicolumn{3}{|c|}{$1.73 \times 10^{-9}$} \\
\hline
\end{tabular}

Table 4-4. Numerical results - Effective diffusion coefficients. The values were the slopes of the linear regression lines on the plots of the scaled average FPT vs. the squared displacement.

\begin{tabular}{|c|c|c|c|c|c|c|}
\hline \multicolumn{7}{|c|}{$\begin{array}{l}\text { Numerical Estimate of Effective Diffusion Coefficients }\left[\mathrm{cm}^{2} / \mathrm{sec}\right] \\
\qquad \mathbf{x}^{2}=D_{\text {eff }}\left(2<t>+k<t>^{3}\right)\end{array}$} \\
\hline \multirow{2}{*}{$\begin{array}{c}\text { Simulation } \\
\text { Trial \# }\end{array}$} & \multicolumn{3}{|c|}{ SRW } & \multicolumn{3}{|c|}{ KW } \\
\hline & 1 & 2 & 3 & 1 & 2 & 3 \\
\hline $\mathbf{D}_{\text {eff }}$ & $6.97 \times 10^{-9}$ & $7.53 \times 10^{-9}$ & $6.77 \times 10^{-9}$ & $7.23 \times 10^{-9}$ & $7.23 \times 10^{-9}$ & $7.42 \times 10^{-9}$ \\
\hline $\mathbf{r}^{2}$ & 0.999 & 0.999 & 0.999 & 0.998 & 0.998 & 0.999 \\
\hline Average & & $7.09 \times 10^{-9}$ & & & $7.29 \times 10^{-9}$ & \\
\hline S.D. & & $0.40 \times 10^{-9}$ & & & $0.11 \times 10^{-9}$ & \\
\hline
\end{tabular}




\section{Index of Diffusion: Average FPT and Squared Axial Displacement Relationships}

A numerical description of the character of the random motions of the beads and the red cells was assigned by fitting power law regressions to plots of the average first passage time vs. the squared axial displacement $\left(\mathrm{x}^{2}=\mathrm{c}<\mathrm{t}>^{\alpha}\right)$. Figure 4-12 shows the plots of the experimental data and table 4-5 shows the values of the exponent $\alpha$ for each experimental trial. Figure 4-13 shows the plots of the numerically simulated data and table 4-6 shows the values of the exponent $\alpha$ for each numerical trial. As expected the exponent alpha was around 3 for the bead trials. The red cell trials had an average exponent of 2.85 indicating a dispersive process. The exponent alpha was around 3 for the SRW simulation and that was expected for a process that is simulating Brownian motion. The KW simulation exponent (2.79) was closer to the RBC value and provided an indication that dispersion can be modeled using a persistent random walk.

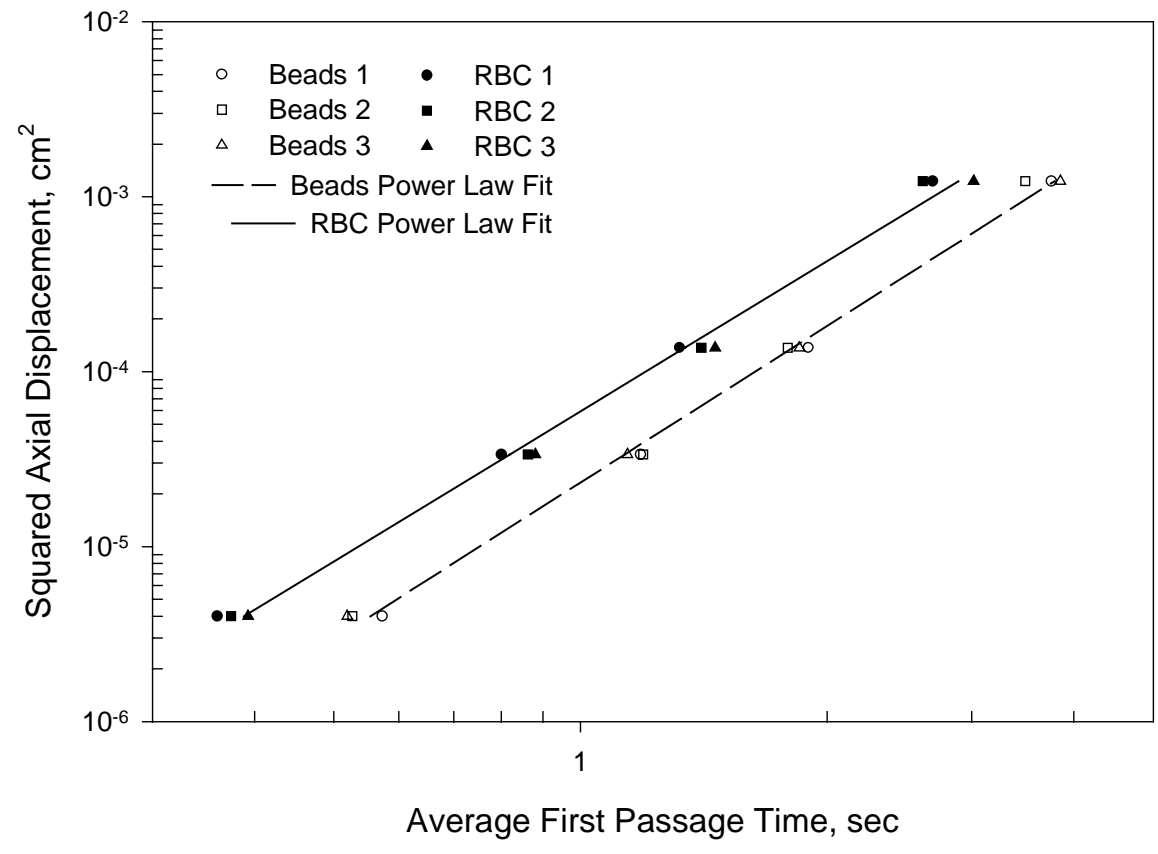

Figure 4-12. Experimental results - The character of diffusion was estimated by the exponent of the power law fit on the plot of the average FPT vs. the squared axial displacement. The data are represented by the symbols and the lines represent the regression fits. 
Table 4-5. Experimental Results - The character of diffusion. Values were estimated quantitatively by the exponent of the power-law regression fit on the plot of the average FPT vs. the squared axial displacement (figure 4-12).

\begin{tabular}{|c|c|c|c|c|c|c|}
\hline \multicolumn{7}{|c|}{$\begin{array}{l}\text { Estimate of the diffusion character } \\
\qquad \mathbf{x}^{2}=\mathbf{c}<\boldsymbol{t}>^{\alpha}\end{array}$} \\
\hline \multirow{2}{*}{$\begin{array}{c}\text { Experiment } \\
\text { Trial \# }\end{array}$} & \multicolumn{3}{|c|}{ Beads } & \multicolumn{3}{|c|}{ RBC } \\
\hline & 1 & 2 & 3 & 1 & 2 & 3 \\
\hline Coefficient (c) & $2.08 \times 10^{-5}$ & $2.45 \times 10^{-5}$ & $2.47 \times 10^{-5}$ & $6.80 \times 10^{-5}$ & $6.08 \times 10^{-5}$ & $5.13 \times 10^{-5}$ \\
\hline Exponent $(\alpha)$ & 3.0390 & 3.0347 & 2.8565 & 2.8247 & 2.9217 & 2.8021 \\
\hline \multirow[t]{2}{*}{$\mathbf{r}^{2}$} & 0.999 & 0.995 & 0.999 & 0.998 & 0.993 & 0.998 \\
\hline & \multicolumn{6}{|c|}{ Power law fit through all data points } \\
\hline Coefficient (c) & & $2.32 \times 10^{-5}$ & & & $5.94 \times 10^{-5}$ & \\
\hline Exponent $(\alpha)$ & & 2.9759 & & & 2.8484 & \\
\hline $\mathbf{r}^{2}$ & & 0.999 & & & 0.997 & \\
\hline
\end{tabular}

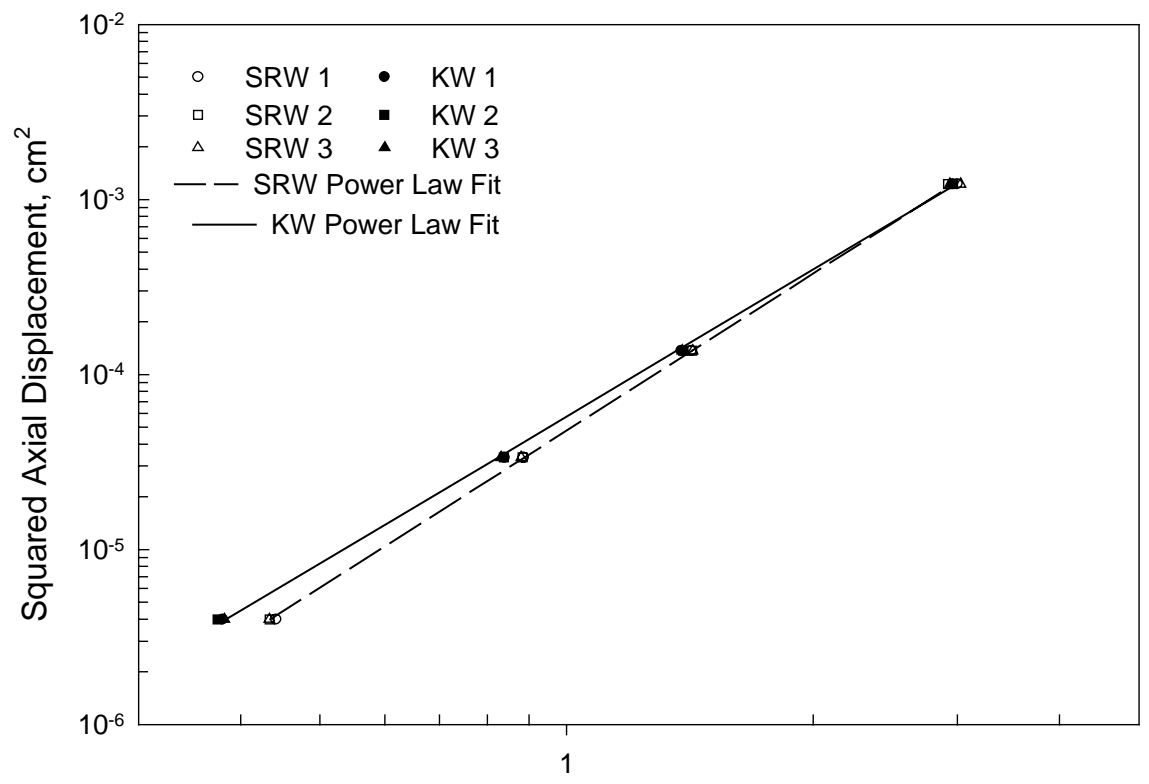

Average First Passage Time, sec

Figure 4-13. Numerical results (as in figure 4-12) 
Table 4-6. Numerical results - The character of diffusion. Values were estimated quantitatively by the exponent of the power-law regression fit on the plot of the average FPT vs. the squared axial displacement (figure 4-13).

\begin{tabular}{|c|c|c|c|c|c|c|}
\hline \multicolumn{7}{|c|}{$\begin{array}{l}\text { Estimate of the diffusion character } \\
\qquad \mathbf{x}^{2}=\mathbf{c}<\mathrm{t}>^{\alpha}\end{array}$} \\
\hline \multirow{2}{*}{$\begin{array}{c}\text { Experiment } \\
\text { Trial \# }\end{array}$} & \multicolumn{3}{|c|}{ SRW } & \multicolumn{3}{|c|}{ KW } \\
\hline & 1 & 2 & 3 & 1 & 2 & 3 \\
\hline Coefficient (c) & $4.71 \times 10^{-5}$ & $4.86 \times 10^{-5}$ & $4.77 \times 10^{-5}$ & $5.73 \times 10^{-5}$ & $5.77 \times 10^{-5}$ & $5.75 \times 10^{-5}$ \\
\hline Exponent $(\alpha)$ & 2.9858 & 2.9979 & 2.9416 & 2.7870 & 2.7683 & 2.8026 \\
\hline \multirow[t]{2}{*}{$\mathbf{r}^{2}$} & 0.999 & 0.999 & 0.999 & 0.999 & 0.999 & 0.999 \\
\hline & \multicolumn{6}{|c|}{ Power law fit through all data points } \\
\hline Coefficient (c) & & $4.78 \times 10^{-5}$ & & & $5.75 \times 10^{-5}$ & \\
\hline Exponent $(\alpha)$ & & 2.9749 & & & 2.7860 & \\
\hline $\mathbf{r}^{2}$ & & 0.999 & & & 0.999 & \\
\hline
\end{tabular}




\section{CHAPTER 5. DISCUSSION}

Previous studies have shown enhanced transport of cells and solutes in the presence of red blood cells (44 (references therein). Properly characterizing the random motions of cells in flow in the vasculature or an artificial conduit is important because of the associated biophysical implications with clinical significance. In this work, experiments with erythrocytes were conducted in a simplified in vitro model of red cell transport. The channel height $(\sim 50$ micrometers) was in the order of magnitude of vessels in the microvasculature. The analysis was performed within a theoretical framework that included probabilistic concepts. The work characterized the random motions of human erythrocytes flowing in concentrated suspensions and used dilute suspension flows of 0.46-micrometer diameter beads to provide a simpler, better understood physical standard as a control.

The experimental method is similar to the one used by Cerda and van de Ven to study bead doublets (6). The use of a constant stage speed in this method is the principal difference between the methods. This condition of constant stage speed allowed measurements to be restricted to a narrow lateral region, which has nearly constant values of average concentration and shear rate. Though this is a simple modification of their method, it allows for the study of events at specific regions across the entire channel or tube where concentration and shear vary significantly. For example, near the wall, where cellular concentrations vary with cell types (1; 37), and near the center, where the average shear rate is zero. The center region is also interesting because the fluctuating shear rate is likely to be significant, and the concentration of particles is highest (26). 
The experimental method also gives an indirect way to consider motions that move individual particles along concentration and shear gradients (2). The particles were tracked from the point they changed their axial motion. That event was presumably associated with a continuing lateral motion across the flow plane that corresponded to the speed of the stage. The reader is referred to figure 3-6 for a schematic representation of the velocity profiles in the moving reference frame. Particles that were moving from left to right in the video screen and then changed their axial motion (q-type) presumably were moving laterally deeper into the channel. These motions can be considered as motions down a shear gradient and up a concentration gradient. The opposite would hold for p-type cells, which moved from left to right before they changed their axial direction. In these studies, there was no significant difference in the results between the p-type and q-type particles, which indicates that the particles were exposed to a local environment with a relatively constant concentration and shear gradient.

The irregular motions were assumed to begin in the lateral direction and to have associated events in the axial direction, which could be observed and simulated by numerical methods. By studying the particle motions in the moving reference frame, the erratic motions of the particles were easily observed as the particle presumably changed lateral position and hence axial speed. This assumption is consistent with the concept of a low particle Reynolds number, which implies that the viscous stresses outweigh those due to inertia, and so the particle will stay at the speed of the surrounding fluid. This model is limited and true to the extent that the dynamic "structure" in the concentrated suspension and its associated effects are ignored. For example if a marked particle is a part of a rouleaux that is exposed to high shear gradient, it may temporarily tumble and turn faster than if it followed the local fluid velocity. True rouleaux would be rare in these experimental trials since the red cells were washed and the shear rate was 
in the range that does not favor rouleaux formation. But the temporary formation and disbanding of cells groups is to be expected; such flow induced particle associations are a fundamental part of the viscosity theory advanced by Quemada (33).

An aspect warranting further research is the role of the channel wall as a restrictive boundary. In other words, would the particles behave differently if they were free to flow without feeling the presence of the wall? In the case of the bead experiments, the channel height to the particle diameter ratio was in the order of 100 , and beads were tracked when they were approximately 30 particle diameters away from the wall. In the case of the red cells that ratio was around 20 and cells were tracked when they were approximately four to five cell diameters away from the wall. The boundary problem though is not simple if the role of concentration is considered. Is the channel wall more important than the presence of another cell adjacent to the one being tracked? Perhaps a study of red cells in dilute suspension would provide some answers, but such a study is as likely to shed light on the additional issue of red cell flexibility and the tendency of such bodies to center themselves in a flow channel. This is in contrast to the tendency of rigid particles to move to places midway between the centerline and the wall (also known as the Segre-Silberberg effect) (22).

As noted above the low particle Reynolds number required that the observed axial motions be associated with changes in the particle's lateral position, which were presumed to occur first for the numerical models of suspension events in two dimensions. The simulations focused in the differences of the results when the lateral motions were modeled by two different random walks; a simple random walk that is commonly used to model diffusion and a persistent random walk that is seldom used. The simple random walk predicted the values as "diffusive" and the persistent random walk (Kac walk) was in good agreement with the experimental RBC 
values. There were no significant differences in the first passage time distributions between the results obtained by the two different models. Only the Kac walk provided a means to model the fraction of particles that crossed the fences in the same direction as their initial direction of motion, as shown in figure 4-10.

\section{Comparisons of Results with Published Values}

Experimental data were fitted to mathematical and probabilistic models to estimate the effective diffusion coefficients and to distinguish between dispersive and diffusive motions. The resulting values for the diffusion coefficients compared well with published values and served as a way to validate the experimental protocols. Goldsmith and coworkers measured the diffusion coefficient of red cells and obtained values in the order of $1 \times 10^{-8} \mathrm{~cm}^{2} / \mathrm{sec}(20)$. The values estimated by this work were in the order of $0.9 \times 10^{-8} \mathrm{~cm}^{2} / \mathrm{sec}$. Cerda and van de Ven measured the diffusion of bead doublets in dilute suspensions and their value was in the order of $3 \times 10^{-9}$ $\mathrm{cm}^{2} / \mathrm{sec}$ (6). The values estimated by this work, for single beads, were in the order of $4 \times 10^{-9}$ $\mathrm{cm}^{2} / \mathrm{sec}$ and compares well to the value calculated with the Stokes-Einstein equation, $4.4 \times 10^{-9}$ $\mathrm{cm}^{2} / \mathrm{sec}$.

Using the time and squared displacement relationships described in the probabilistic CTRW model developed by Compte $\left(\mathrm{x}^{2} \sim \mathrm{t}^{\alpha}\right)(10)$, the motions of RBC and beads were characterized as dispersive and diffusive, respectively. Such methods are widely accepted in the statistical physics community. To our knowledge, this is the first time that the red cell motions have been described by use of such quantitative methods.

For the RBC, the values of the time exponent in the Compte relationship were consistently less than 3 with an average value of 2.84 . The average of the beads was 2.97 . Two 
of the bead trials produced exponents higher than 3 and the third one was less than 3 ; the value less than 3 was believed to have been influenced by the observed adhesion of beads on the channel walls, which was not a significant event in the other trials.

Given the small number of trials for each event (3) and the small number of observations, the difference in the time exponent values between the beads and the RBC is too small to be statistically significant. Thus, additional experiments are needed. But according to the accepted notion of the dispersive behavior of red cells and the theoretical relationships developed by Compte, the red cell exponents are probably representative of anomalous diffusion or dispersive process in these simple suspension studied here.

\section{Effects of the Fluorescent Labels}

The physical and mechanical properties of cells play an important role in the way cells behave in suspensions (7). It should be noted that the cells tracked were stained with two different dyes and washed several times with buffers. In this study, no attempt was made to quantitatively determine any changes in their physical characteristics or mechanical properties, but the cells were examined under high magnification (40x) and their biconcave shape and plasma membrane were seemingly intact.

The membrane dye, DiI, which could presumably cause some changes in the mechanical properties of the cell's membrane, has been used to stain cells that were injected into animals for microcirculation studies (34). In these studies (personal communication), no rapid decay in the number of the stained cells was observed that could perhaps indicate rapid uptake of the stained cells in the reticuloendothelium. The cytoplasmic dye, Cell Tracker Orange was assumed to be 
innocuous since it has been used to study events in cellular differentiation (35), a process that would be easily disrupted if structural or chemical changes were caused by this dye.

\section{Sources of Measurement Error}

The net accuracy of the calculated parameters in this work was subject to the accuracy and precision of the following: 1) the time resolution that was set by the NTSC video standard $\left(1 / 30^{\text {th }}\right.$ second $\left.) ; 2\right)$ the distance calibration of the video pixels; 3$)$ deviation of the fluctuations in the stage speed from the ideal steady value; 4) fluctuations of the flow rate about the steady value, which could occur due to both hardware and events in the channel, such as sticking of particles or bubbles to the walls upstream or downstream of the sections imaged by the camera; and 5) treating the velocity profile in the glass channel as parabolic.

Since the time measurement were based on image analysis of videotape recordings, they were subject to a widely accepted video standard (NTSC) and the resolution of the measurements could not be improved beyond $1 / 30^{\text {th }}$ of a second. The distance calibration was subject to the system's video resolution (640 horizontal pixels by 480 vertical pixels) after recording the signal. Each pixel was calibrated to be 1.667 micrometers per pixel and any errors in distance measurement would be associated with the distance value assigned to each pixel with the use of a microscope micrometer as the distance standard. The first passage time measurement started when a particle changed its axial direction and ended when it crossed a fence. The fence distances were always a fixed number of pixels. The point in space or time at which the particle turned around was not known exactly and the time at which the particle center first crossed over the fence was not known exactly either. Assuming that the errors in position and time can be lumped together an estimate of the error of the time measurements can be obtained. Following 
the traditional method, the resolution error would be $1 / 2$ the minimum time resolution, in this case the minimum time resolution was one video frame. The estimation of error given here has assumed that the resolution error was one rather than half a video frame and a box plot graph with the percentage error in each measurement is shown in figure 5-1.

The stage speed was accurate to within $1 \%$ of the set value, according to manufacturer's specifications. Since the stage used in the experiments was designed to move to specific coordinates with submicrometer accuracy, that claim seemed valid. The speed of the stage was not measured to check for variations between individual or small numbers of video frames since available equipment was not sufficiently precise.

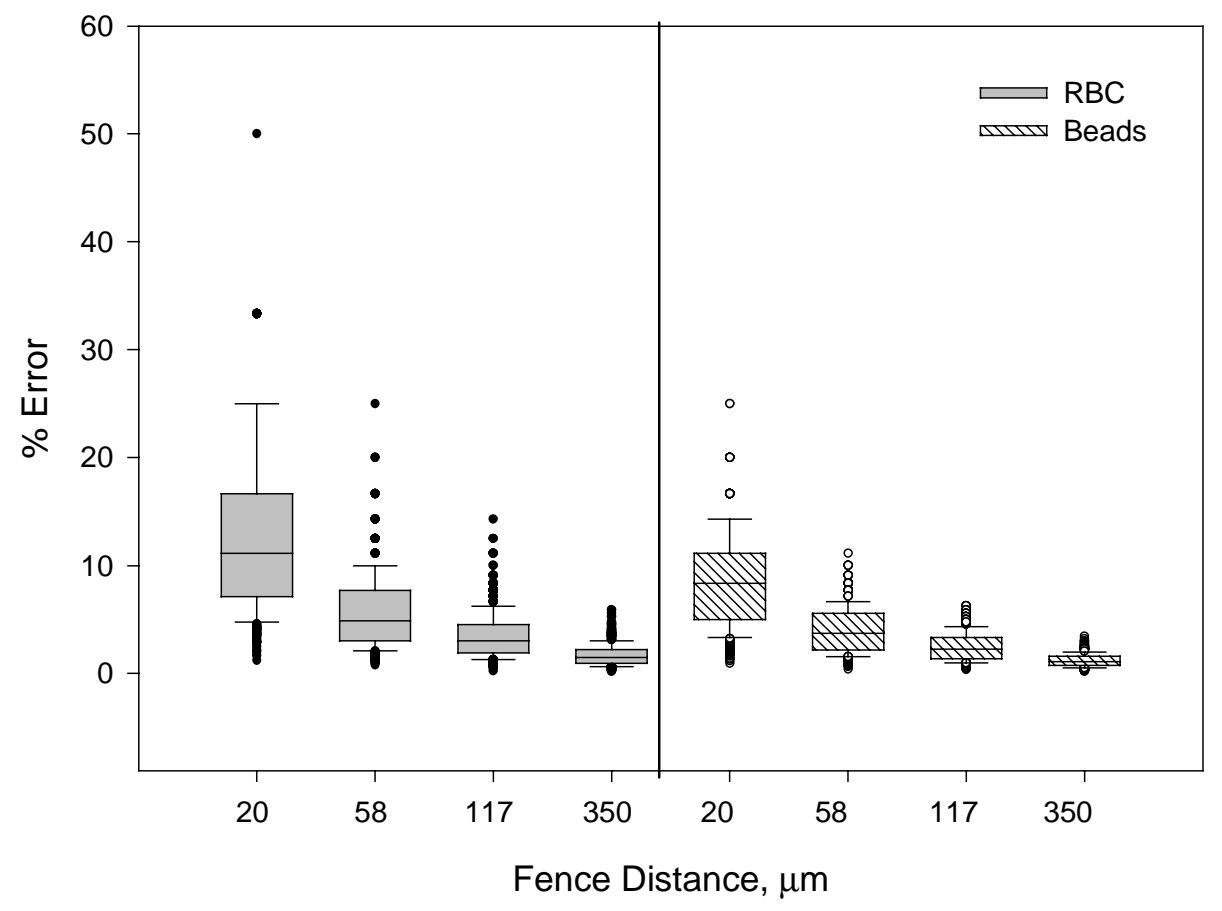

Figure 5-1. Box plots for the time measurement error. The error was calculated as one thirtieth of a second (the time resolution) divided by the first passage time. 
The pump flow rate was also accurate to within $1 \%$, according to manufacturers' specifications, for both types of pumps used in the experiments. The pump in the RBC experiments was stationed on the microscope table and was connected to the glass channel with a piece of tubing that was allowed to move freely as the stage traveled back and forth. Refer to figure 3-2 for a schematic representation of the microscope stage and flow assembly. The tubing was long and was hanging below the level of the microscope stage. The effect of the moving section of tubing was tested by allowing cells to settle in the tube. The cells were then observed after the stage had been moving back and forth a few times. There was no observed motion to the stationary cells and they started flowing readily when the tubing was raised above the microscope stage level or the syringe pump was turned on.

The measurements presented here were precise in the sense of being reproducible, but were not accurate in an absolute sense or in the sense of primary standards. In both the dilute and concentrated suspension trials the flow was assumed to be parabolic. For the beads the assumed flow profile was more accurate since the dilute suspension more closely followed Poiseuille flow, which predicted the maximum velocity to be 2500 micrometers per second given the geometry of the channel and the flow rate. For the red cells there was probably some blunting in the average velocity profile (20). But, as it was ascertained from preliminary studies that tracked particles when the stage moved with a speed of around 2400 micrometers per second, the center velocity was not blunted to a great extent because particles were still moving faster than the stage. That result is also consistent with the minimum degree of blunting expected from the $25 \%$ reservoir hematocrit (9). The channel hematocrit was lower than the feed reservoir hematocrit due to the Fahraeus effect (19). Using a published formula the channel hematocrit was estimated 
to be approximately $19 \%$ assuming that Barbee and Cokelet's formula for calculating the tube hematocrit is valid for channels (3).

The values for the beads are probably accurate within $10 \%$. The values for the red cells are accurate to the extent that the Foister and ven de Ven's formula, derived assuming convection diffusion as the mode of lateral transport, can be applied to the dispersive motions of the red cells. In any case, both the bead and RBC values compare favorably with published values obtained by different experimental and theoretical methods.

\section{Implications of the Numerical Results}

A difference in the exponents that determined the diffusive and dispersive behaviors was noted in the simulations. The exponent of the time in the Compte's relationship was 2.97 for the simple random walk and 2.79 for the Kac walk. The implication of the later result is that a different mathematical model may be more appropriate to model the events in particle suspensions and the derivation of the Telegraph equation from the Kac walk provides a starting point for such a model.

The balanced fractions of $\mathrm{p}$ - and q-type particles crossing a fence in the same direction as their initial motion (cf. figure 4-8) indicated that the particles remained close to their initial lateral position and were not exposed to the entire parabolic flow. This assumption was confirmed further by results of the numerical simulations that showed that particles clustered within a red cell diameter from their initial lateral position. 


\section{CHAPTER 6. CONCLUSIONS AND FUTURE WORK}

\section{Conclusions}

1. The experimental method is capable of estimating effective diffusion coefficients in suspension flows of varying concentration.

2. The method is capable of making diffusion estimates in local regions within the suspension.

3. The method is expected to have the capacity to distinguish between diffusion and dispersion by means of the Compte exponent.

\section{Future Work}

Future work will focus on additional experiments studying regions across the channel height. In addition, the effects of wall shear rate, hematocrit, and wall roughness will be investigated. Improved data reduction methods will be sought to speed the data analysis. Methods that will automatically track single or multiple particles will be developed to speed data reduction. Additional work will focus on the analysis of the first passage time distributions to extract information through probabilistic analysis. 


\section{LIST OF REFERENCES}




\section{LIST OF REFERENCES}

1. Aarts, P.A., S.A. van den Broek, G.W. Prins, G.D. Kuiken, J.J. Sixma, and R.M. Heethaar. 1988. Blood platelets are concentrated near the wall and red blood cells, in the center in flowing blood. Arterioscl. 8:819-824.

2. Acrivos, A. 1995. Bingham award lecture - 1994. Shear - induced particle diffusion in concentrated suspensions of non-colloidal particles. J. Rheol. 39:813.

3. Barbee, J.H., and G.R. Cokelet. 1971. The Fahraeus effect. Microvasc. Res. 3:6-16.

4. Bird, B.L., W.E. Stewart, and E.N. Lightfoot. 1960. Transport phenomena. John Wiley \& Sons, New York. 94-96.

5. Brash, J.L., J.M. Brophy, and I.A. Feuerstein. 1976. Adhesion of platelets to artificial surfaces: effect of red cells. J. Biomed. Mater. Res. 10:429-443.

6. Cerda, C. M., and T. G. M. van de Ven. 1983. Translational diffusion of axysymmetrical particles in shear flow. J. Colloidal Interface Sci. 93:54-62.

7. Chien, S.S., R.J. Usami, and M.I. Gregersen. 1967. Blood viscosity: influence of erythrocyte deformation. Science. 157:827-829.

8. Chien, S.S., R.J. Usami, and R. Skalak. 1984. Blood flow in small tubes. In Handbook of physiology. American Physiologic Society, Bethesda. 217-249.

9. Cokelet, G.R. 1980. Rheology and hemodynamics. Ann. Rev. Physiol. 42:311-324.

10. Compte, A. 1997. Continuous time random walks on moving fluids. Phys. Rev. E. 55:68216831.

11. Cussler, E.L. 1997. Diffusion: Mass Transfer in Fluid Systems. Cambridge University Press, Cambridge. 81-86.

12. Eckstein, E. C., D. G. Bailey, and A. H. Shapiro. 1977. Self-diffusion of particles in shear flow of a suspension. J. Fluid Mech. 79:191-208.

13. Evans, M., N. Hastings, and B. Peacock. 1993. Statistical distributions. John Wiley \& sons, New York. 8-126. 
14. Foister, R. T., and T. G. M. van de Ven. 1980. Diffusion of Brownian particles in shear flow. J. Fluid Mech. 96:105-132.

15. Gardiner, C.W. 1985. Handbook of Stochastic Methods. Springer Verlag, New York. 1-437.

16. Gillespie, D.T. 1992. Markov Processes: An Introduction for Physical Scientists. Academic Press, Boston. 48-58.

17. Goldsmith, H.L. 1967. Microscopic flow properties of red cells. Fed. Proc. 26:1813-1820.

18. Goldsmith, H.L. 1971. Red cell motions and wall interactions in tube flow. Fed. Proc. 30:1578-1590.

19. Goldsmith, H.L., G.R. Cokelet, and P. Gaehtgens. 1989. Robin Fohraeus: evolution of his concepts in cardiovascular physiology. Am. J. Physiol. 257:H1005-H1015

20. Goldsmith, H. L., and J. C. Marlow. 1979. Flow behavior of Erythrocytes II. Particle motions in concentrated suspensions of ghost cells. J. Colloidal Interface Sci. 71:383-407.

21. Goldsmith, H.L., and S. Spain. 1984. Margination of leukocytes in blood flow through small tubes. Microvasc. Res. 27:204-222.

22. Goldsmith, H.L., and V.T. Turitto. 1986. Rheological aspects of thrombosis and haemostasis: basic principles and applications. ICTH-Report--Subcommittee on Rheology of the International Committee on Thrombosis and Haemostasis. Thromb. Haemost. 55:415-435.

23. Hersh, R., and R. J. Griego. 1969. Brownian motion and potential theory. Sci. American. 220:66-74.

24. Kac M. 1974. A stochastic model related to telegrapher's equation. Rocky Mountain J. Math. 4:497-509.

25. Klafter J., M. F. Shlesinger, and G. Zumofen. 1996. Beyond Brownian motion. Phys. Today. 49:33-39.

26. Koh, C. J., P. Hookam, and L. G. Leal. 1994. An experimental investigation of concentrated suspension flows in a rectangular channel. J. Fluid Mech. 266:1-32.

27. Leighton, D., and A. Acrivos. 1987. Measurement of shear-induced self-diffusion in concentrated suspensions of spheres. J. Fluid Mech. 177:109-131. 
28. Montroll, E.W., and B.J. West. 1979. On an enriched collection of stochastic processes. In Fluctuation phenomena. E.W. Montroll and J.L. Lebowitz, editors. North-Holland Publishing Co., New York. 61-175.

29. Munn, L.L., R.J. Melder, and R.K. Jain. 1996. Role of erythrocytes in leukocyte-endothelial interactions: mathematical model and experimental validation. Biophys. J. 71:466-478.

30. Nobis, U., A.R. Pries, G.R. Cokelet, and P. Gaehtgens. 1985. Radial distribution of white cells during blood flow in small tubes. Microvasc. Res. 29:295-304.

31. Nott, P. R., and J. F. Brady. 1994. Pressure-driven flow of suspensions: simulations and theory. J. Fluid Mech. 275:157-199.

32. Phillips, R.J., R.C. Armstrong, R.A. Brown, A. Graham, and J.R. Abbott. 1992. A constitutive model for concentrated suspensions that accounts for shear-induced particle migration. Phys. Fluids. A. 4:30-40.

33. Quemada, D. 1993. A non-linear Maxwell model of biofluids: application to normal blood. Biorheology. 30:253-265.

34. Roth, N.M., M.R. Sontag, and M.F. Kiani. 1999. Early effects of ionizing radiation on the microvascular networks in normal tissue. Radiat. Res. 151:270-277.

35. Ruiz, M., S.T. Roodman, J.D. Bouhasin, and A.P. Knutsen. 1996. T cell differentiation/maturation of CD34+ stem cells from HIV-seropositive hemophiliacs in cultured thymic epithelial fragments. Stem Cells. 14:132-145.

36. Schmid-Schoenbein, G.W., and B.W. Zweifach. 1975. RBC velocity profiles in arterioles and venules of the rabbit omentum. Microvasc. Res. 10:153-164.

37. Tangelder, G.J., D.W. Slaaf, A.M. Muijtjens, T. Arts, M.G.A. oude Egbrink, and R.S. Reneman. 1986. Velocity profiles of blood platelets and red blood cells flowing in arterioles of the rabbit mesentery. Circ. Res. 59:505-514.

38. Taylor, G. 1953. Dispersion of soluble matter in solvent flowing slowly through a tube. Proc. Royal Soc. A219:186-203.

39. Taylor, G. 1954. Conditions under which dispersion of a solute in a stream of solvent can be used to measure molecular diffusion. Proc. Royal Soc. A225:473-477.

40. Tortora, G.J., and S.R. Grawbowski. 1996. The cardiovascular system: The blood. In Principles of anatomy and physiology. Harper Collins, New York. 552-577. 
41. Turitto, V. T., A. M. Benis, and E. F. Leonard. 1972. Platelet diffusion in flowing blood. Ind. Eng. Chem. Fund. 11:216-223.

42. Unthank, J.L., J.M. Lash, J.C. Nixon, R.A. Sidner, and H.G. Bohlen. 1993. Evaluation of carbocyanine-labeled erythrocytes for microvascular measurements. Microvasc. Res. 45:193-210.

43. Weiss, G.H. 1994. Aspects and applications of the random walk. North-Holland, New York. $1-115$.

44. Zydney, A. L., and C. K. Colton. 1988. Augmented solute transport in the shear flow of a concentrated suspension. Physicochem. Hydrod. 10:77-96. 
APPENDIX 


\title{
APPENDIX
}

\section{Simulation Code $(\mathrm{C}++)$}

\section{Simple Random Walk}

\author{
// $\mathrm{C}++$ header files, libraries \\ \#include $<$ stdio.h $>$ \\ \#include $<$ iostream.h $>$ \\ \#include $<$ math.h $>$ \\ \#include $<$ time.h $>$ \\ \#include $<$ stdlib.h $>$ \\ \#include $<$ fstream.h $>$ \\ \#include $<$ iomanip.h $>$ \\ // Main program function \\ int main(void) \\ \{
}

// Initialize and seed the uniform random number generator

srand( (unsigned)time( 0 ));

// Declare Variables

double particle_count; double height; double init_lateral_position; int count; double time; double lateral_position; double axial_position; double axial_motion; double particle_motion; double lateral_step; double time_step; double move; double fence_distance; double upper_wall; double lower_wall; double stage_motion; double particle; int initial_direction; int lateral_pointer; double max_velocity; double flow_rate; double width; int fence_count; double stage_speed; char filename[20]; double a; double b; double c; double d;

\section{// Initialize Variables}

$\begin{array}{ll}\mathrm{a}=20 ; \mathrm{b}=58 ; \mathrm{c}=117 ; \mathrm{d}=350 ; & \text { // Set four fence distances, microns } \\ \text { particle_count=1700; } & \text { // Simulate this many particles } \\ \text { height=54; } & \text { // Channel dimensions, microns } \\ \text { width=556; } & \text { // Syringe pump flow rate, } \mathrm{ml} / \mathrm{hr} \\ \text { flow_rate=0.18; } & \text { // Stage speed, microns/second } \\ \text { stage_speed=1786; } & \text { // Moving reference frame position, microns } \\ \text { init_lateral_position }=14.41 ; & \text { // Time increment, seconds } \\ \text { time_step=0.0165; } & \text { // Lateral motion step size, microns } \\ \text { lateral_step=0.3; } & \\ \text { upper_wall =height/2; } & \text { // Set boundaries (height) } \\ \text { lower_wall }=(-h e i g h t) / 2 ; & \text { // Set efflux direction (arbitrarily) } \\ \text { initial_direction=1; } & \text { // Maximum velocity, microns/second }\end{array}$


$\max \_$velocity $=1.5 *$ flow_rate $/ 3600 /$ height $/$ width $/ 1 \mathrm{e}-12$;

cout $<<$ "Enter data file name: "; $\operatorname{cin}>>$ filename; ofstream fout; fout.open(filename);

fout $<<$ filename $<<" \backslash n \backslash n$ "; fout $<<$ "time_step" $<<" \backslash t$ " $<<$ time_step $<<" \backslash n "$;

fout $<<$ "lateral_step" $<<" \backslash t "<<$ lateral_step $<<" \backslash n \backslash n \backslash n \backslash n "$;

fout $<<" \mid \mathrm{t}$ " $<<\mathrm{a}<<$ " um" $<<"|\mathrm{t}| \mathrm{t}$ " $<<\mathrm{b}<<$ " um" $<<"|\mathrm{t}| \mathrm{t}$ " $<<\mathrm{c}<<$ " um" $<<"|\mathrm{t}| \mathrm{t} "<<\mathrm{d}<<$ " um" $<<$ "|n";

for $($ particle $=0 ;$ particle $<$ particle_count;particle $=$ particle +1$)$

\{

// For each particle initialize the following variables

// The initial direction or efflux direction alternates between successive particles

time $=0$; lateral_position $=$ init_lateral_position; axial_position $=0$; count $=0$;

initial_direction=initial_direction*(-1); fence_distance $=\mathrm{a}$; fence_count $=1$;

fout $<<" \backslash n "$;

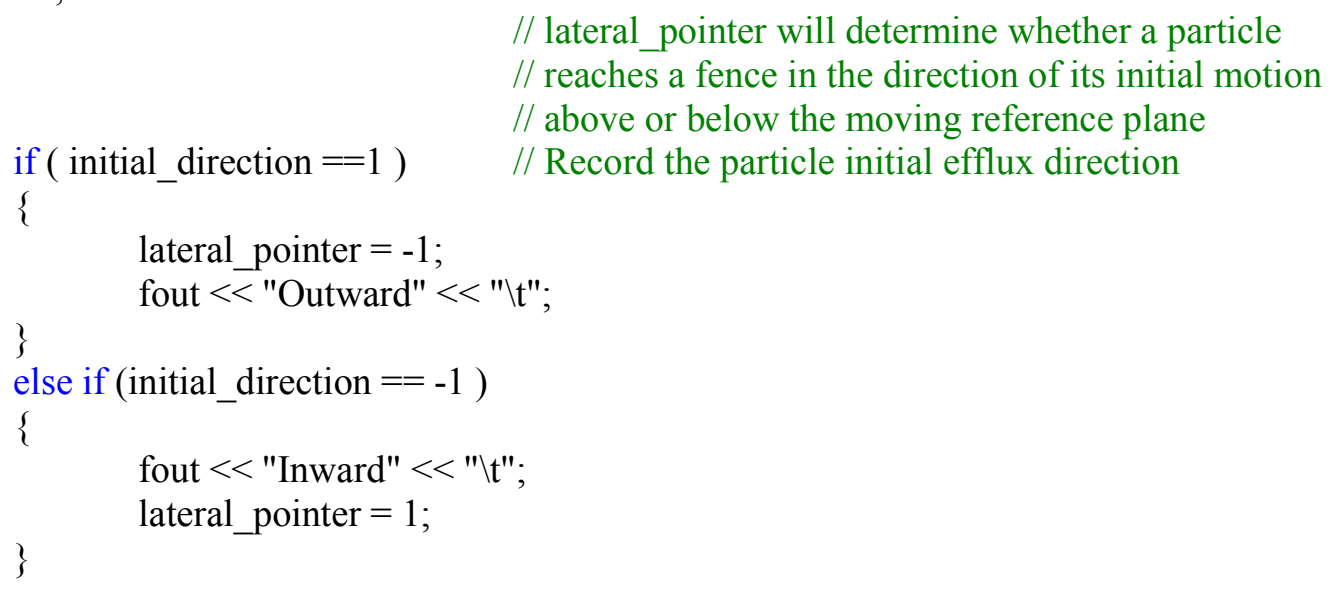

// DO WHILE LOOP - lateral and axial motions

// Position is within plus or minus the current fence distance

do

\{

move $=$ rand ()$; / /$ generate a random number (uniform distribution)

if (move $>=0.5 *$ RAND_MAX)

else

lateral_position $=$ lateral_position + lateral_step;

lateral_position = lateral_position - lateral_step;

// Test the boundary condition and reflect if necessary

// The particle center is assumed to be 2 microns from the wall

if (lateral_position > (upper_wall-2 ))

\{

\} lateral_position $=$ lateral_position - lateral_step;

else if ( lateral_position < (lower_wall+2))

\{

\}

$$
\text { lateral_position = lateral_position + lateral_step; }
$$

// Test the flow symmetry condition and flip lateral_pointer value

// The following two "else if" statements

// deal with the issue of flow symmetry

else if (lateral_position $<$-init_lateral_position $\& \&$ count $==0$ ) 
return 0 ; 


\title{
Kac Random Walk
}

\author{
// $\mathrm{C}++$ header files, libraries \\ \#include $<$ stdio.h $>$ \\ \#include $<$ iostream.h $>$ \\ \#include $<$ math.h $>$ \\ \#include $<$ time.h $>$ \\ \#include $<$ stdlib.h $>$ \\ \#include $<$ fstream.h $>$ \\ \#include $<$ iomanip.h $>$
}

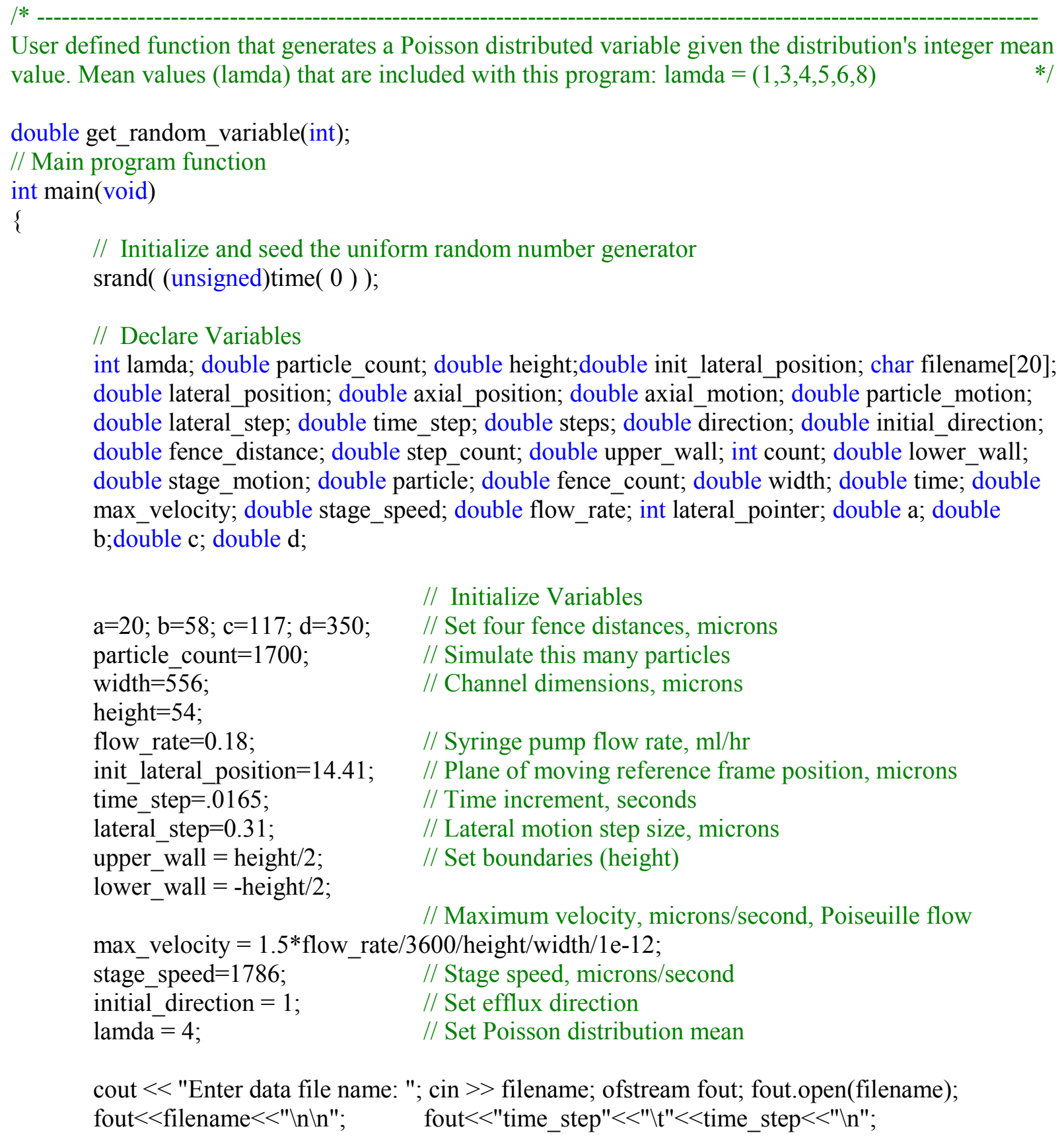




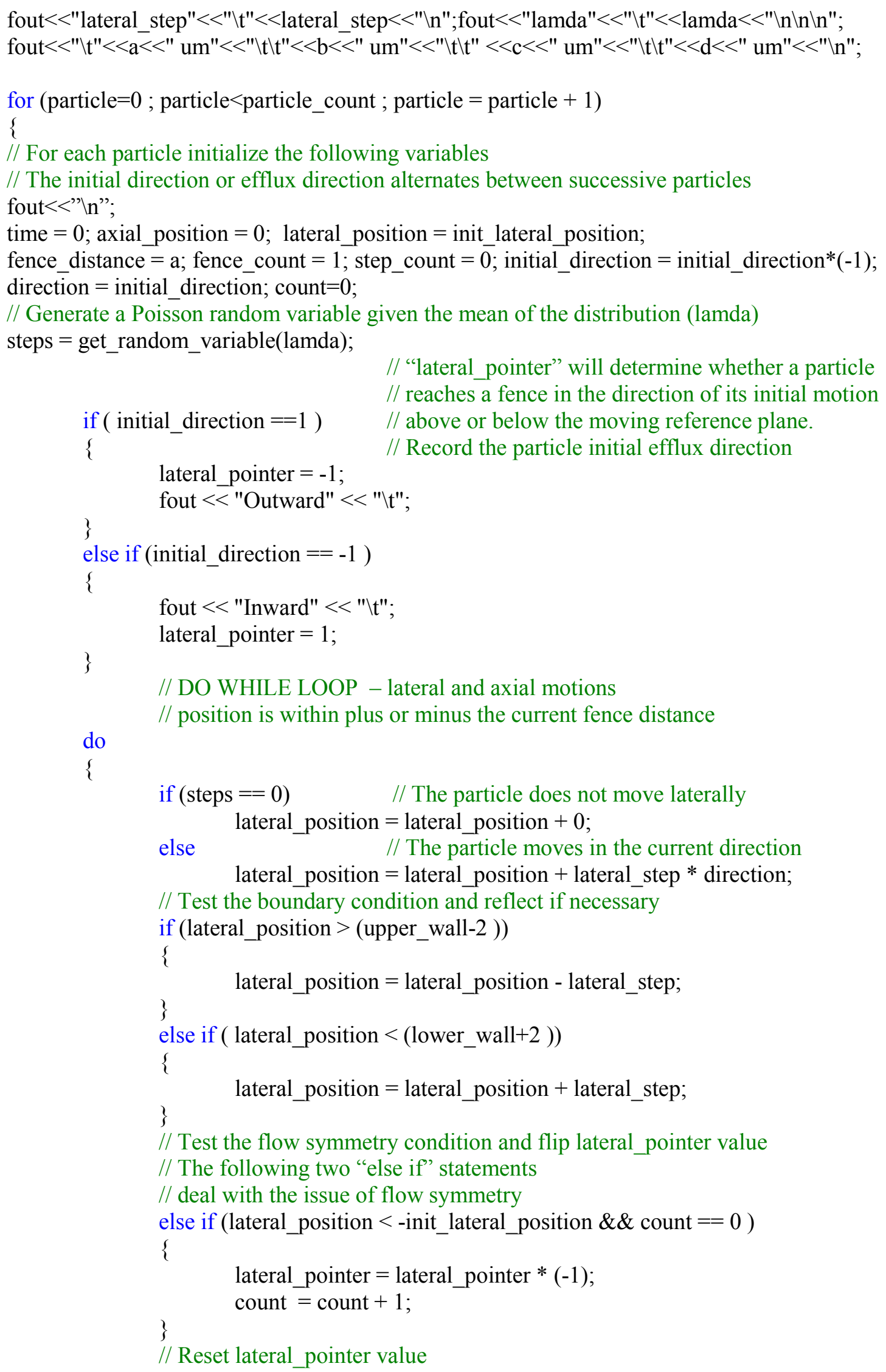




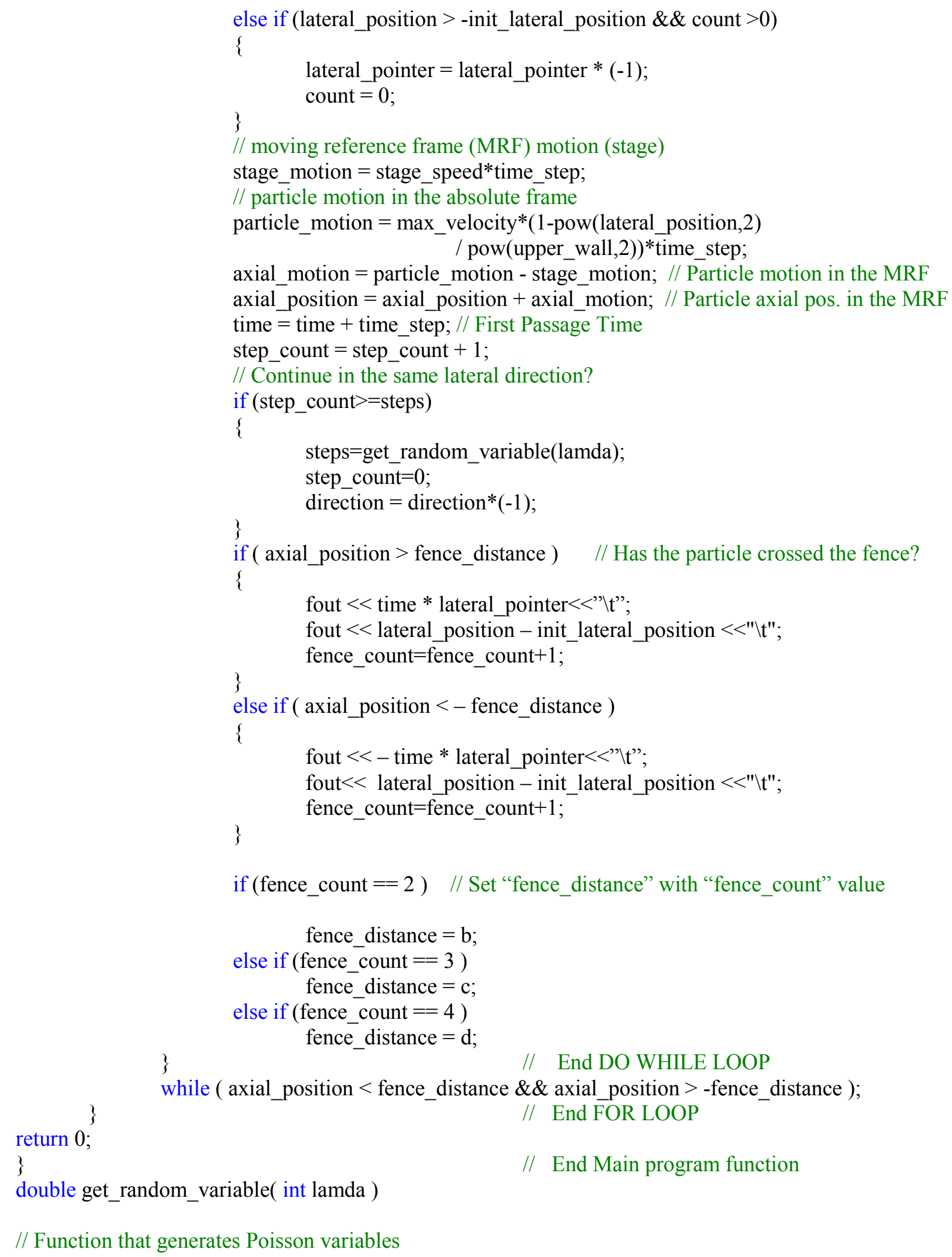

// Function that generates Poisson variables 
/* "random" is the random number generated by the "rand()" function. The "rand () function generates numbers between $0-32767$, the highest number that can be generated is RAND_MAX $=32767$

RAND_MAX is a system variable. "lamda" is the mean and variance that characterizes each distribution of variables generated by the individual if/then modules. "steps" is the value of the single random variable. The uniform pseudorandom number is used to pick a Poisson number, using the inversion technique, from a Poisson Cumulative Distribution

\{

double random; double steps;

random $=\operatorname{rand}()$;

if $($ lamda $==1)$

\{

if (random $>=0 \& \&$ random $<0.36903497 *$ RAND_MAX) steps $=0$;

if (random $>=0.36903497 *$ RAND_MAX \&\& random $<0.73804196 *$ RAND_MAX) steps $=1$;

if (random $>=0.73804196 *$ RAND_MAX \&\& random $<0.91945455^{*}$ RAND_MAX) steps $=2$;

if $\left(\right.$ random $>=0.91945455^{*}$ RAND_MAX $\& \&$ random $<0.98023776 *$ RAND_MAX) steps $=3$;

if $($ random $>=0.98023776 *$ RAND_MAX $\& \&$ random $<0.99632168 *$ RAND_MAX) steps $=4$;

if $($ random $>=0.99632168 *$ RAND MAX $\& \&$ random $<0.99938462 *$ RAND MAX) steps $=5$;

if (random $>=0.99938462 *$ RAND_MAX $\& \&$ random $<0.99994406 *$ RAND_MAX) steps $=6$;

if (random $>=0.99994406 *$ RAND_MAX $\& \&$ random $<=1 *$ RAND_MAX) steps $=7$;

\}

else if (lamda $==2)$

\{

if (random $>=0 \& \&$ random $<0.13564359 *$ RAND_MAX) steps $=0$;

else if (random $>=0.13564359 *$ RAND_MAX \&\& random $<0.40570769 *$ RAND_MAX) steps $=1$;

else if (random $>=0.40570769 *$ RAND_MAX \&\& random $<0.67615769 *$ RAND_MAX) steps $=2$;

else if (random $>=0.67615769 *$ RAND_MAX $\& \&$ random $<0.85670000 *$ RAND_MAX) steps $=3$;

else if (random $>=0.85670000 *$ RAND_MAX \&\& random $<0.94692821 *$ RAND_MAX) steps $=4$;

else if (random $>=0.94692821 *$ RAND_MAX \&\& random $<0.98343590 *$ RAND_MAX) steps $=5$;

else if (random $>=0.98343590 *$ RAND_MAX \&\& random $<0.99538590 *$ RAND_MAX) steps $=6$;

else if (random $>=0.99538590 *$ RAND_MAX \& \& random $<0.99886154 *$ RAND_MAX) steps $=7$;

else if (random $>=0.99886154 *$ RAND_MAX \& \& random $<0.99976795 *$ RAND_MAX) steps $=8$; 
else if (random $>=0.99976795 *$ RAND_MAX \& \& random $<0.99995897 *$ RAND_MAX) steps $=9$;

else if (random $>=0.99995897 *$ RAND_MAX \&\& random $<0.99999103 *$ RAND_MAX) steps $=10$;

else if (random $>=0.99999103 *$ RAND_MAX \&\& random $<=1 *$ RAND_MAX)

steps $=11$;

\}

else if (lamda $==3)$

\{

if (random $>=0 \& \&$ random $<0.04868531 *$ RAND MAX) steps $=0$;

else if (random $>=0.04868531 *$ RAND_MAX $\& \&$ random $<0.19746853 *$ RAND_MAX) steps $=1$;

else if (random $>=0.19746853 *$ RAND_MAX $\& \&$ random $<0.41899301 *$ RAND_MAX) steps $=2$;

else if (random $>=0.41899301 *$ RAND_MAX \&\& random $<0.64436364 *$ RAND_MAX) steps $=3$;

else if (random $>=0.64436364 *$ RAND_MAX \& \& random $<0.81388811 *$ RAND_MAX) steps $=4$;

else if (random $>=0.81388811 *$ RAND_MAX \&\& random $<0.91555245 *$ RAND_MAX) steps $=5$;

else if (random $>=0.91555245 *$ RAND_MAX $\& \&$ random $<0.96653147 *$ RAND_MAX) steps $=6$;

else if (random $>=0.96653147 *$ RAND_MAX $\& \&$ random $<0.98844755 *$ RAND_MAX) steps $=7$;

else if (random $>=0.98844755 *$ RAND_MAX \&\& random $<0.99629371 *$ RAND_MAX) steps $=8$;

else if (random $>=0.99629371 *$ RAND_MAX $\& \&$ random $<0.99879720 *$ RAND_MAX) steps $=9$;

else if (random $>=0.99879720 *$ RAND_MAX \&\& random $<0.99976224 *$ RAND_MAX) steps $=10$;

else if (random $>=0.99976224 *$ RAND_MAX \&\& random $<0.99993007 *$ RAND_MAX) steps $=11$;

else if (random $>=0.99993007 *$ RAND_MAX $\& \&$ random $<0.99997203 *$ RAND_MAX) steps $=12$;

else if (random $>=0.99997203 *$ RAND_MAX \&\& random $<1 *$ RAND_MAX)

steps $=13$;

\}

else if $($ lamda $==4)$

\{

if (random $>=0 \& \&$ random $<0.01833462 *$ RAND_MAX) steps $=0$;

else if (random $>=0.01833462 *$ RAND_MAX \&\& random $<0.09112436 *$ RAND_MAX) steps $=1$;

else if (random $>=0.09112436 *$ RAND_MAX \&\& random $<0.23738333 *$ RAND_MAX) steps $=2$;

else if (random $>=0.23738333 *$ RAND_MAX \&\& random $<0.43290000 *$ RAND_MAX) steps $=3$;

else if (random $>=0.43290000 *$ RAND_MAX \&\& random $<0.62816795 *$ RAND_MAX) steps $=4$;

else if (random $>=0.62816795 *$ RAND_MAX $\& \&$ random $<0.78434231 *$ RAND_MAX) 
steps $=5$;

else if (random $>=0.78434231 *$ RAND_MAX \& \& random $<0.88857692 *$ RAND_MAX) steps $=6$;

else if (random $>=0.88857692 *$ RAND_MAX \&\& random $<0.94857821 *$ RAND_MAX) steps $=7$;

else if (random $>=0.94857821 *$ RAND_MAX \&\& random $<0.97869231 *$ RAND_MAX) steps $=8$;

else if (random $>=0.97869231 *$ RAND_MAX \&\& random $<0.99182564 *$ RAND_MAX) steps $=9$;

else if (random $>=0.99182564 *$ RAND_MAX \&\& random $<0.99714359 *$ RAND_MAX) steps $=10$;

else if (random $>=0.99714359 *$ RAND_MAX $\& \&$ random $<0.99907051 *$ RAND_MAX) steps $=11$;

else if (random $>=0.99907051 *$ RAND_MAX \&\& random $<0.99972821 *$ RAND_MAX) steps $=12$;

else if (random $>=0.99972821 *$ RAND_MAX \&\& random $<0.99993333 *$ RAND_MAX) steps $=13$;

else if (random $>=0.99993333 *$ RAND_MAX \&\& random $<0.99997692 *$ RAND_MAX) steps $=14$;

else if (random $>=0.99997692 *$ RAND_MAX \&\& random $<0.9999961 *$ RAND_MAX) steps $=15$;

else if (random $>=0.9999961 *$ RAND_MAX \&\& random $<1 *$ RAND_MAX) steps $=16$;

\}

else if (lamda $==5$ )

\{

if (random $>=0$ \&\& random $<0.00690897 *$ RAND_MAX) steps $=0$;

else if (random $>=0.00690897 *$ RAND_MAX \& \& random $<0.04033205 *$ RAND_MAX) steps $=1$;

else if (random $>=0.04033205 *$ RAND_MAX \& \& random $<0.12474359 *$ RAND_MAX) steps $=2$;

else if (random $>=0.12474359 *$ RAND_MAX \& \& random $<0.26463718 *$ RAND_MAX) steps $=3$;

else if (random $>=0.26463718 *$ RAND_MAX \&\& random $<0.43995769 *$ RAND_MAX) steps $=4$;

else if (random $>=0.43995769 *$ RAND_MAX \&\& random $<0.61519231 *$ RAND_MAX) steps $=5$;

else if (random $>=0.61519231 *$ RAND_MAX \&\& random $<0.76249615 *$ RAND_MAX) steps $=6$;

else if (random $>=0.76249615 *$ RAND_MAX \&\& random $<0.86704103 *$ RAND_MAX) steps $=7$;

else if (random $>=0.86704103 *$ RAND_MAX \&\& random $<0.93226667 *$ RAND_MAX) steps $=8$;

else if (random $>=0.93226667 *$ RAND_MAX \&\& random $<0.96830000 *$ RAND_MAX) steps $=9$;

else if (random $>=0.96830000 *$ RAND_MAX \&\& random $<0.98633205 *$ RAND_MAX) steps $=10$;

else if (random $>=0.98633205 *$ RAND_MAX \&\& random $<0.99453205 *$ RAND_MAX) steps $=11$;

else if (random $>=0.99453205 *$ RAND_MAX \&\& random $<0.99791410 *$ RAND_MAX) 
steps $=12$;

else if (random $>=0.99791410 *$ RAND_MAX \&\& random $<0.99927821 *$ RAND_MAX) steps $=13$;

else if (random $>=0.99927821 *$ RAND_MAX \&\& random $<0.99974103 *$ RAND_MAX) steps $=14$

else if (random $>=0.99974103 *$ RAND_MAX \&\& random $<0.99992436 *$ RAND_MAX) steps $=15$;

else if (random $>=0.99992436 *$ RAND_MAX \&\& random $<0.99997821 *$ RAND_MAX) steps $=16$;

else if (random $>=0.99997821 *$ RAND_MAX \&\& random $<0.99998846 *$ RAND_MAX) steps $=17$;

else if (random $>=0.99998846 *$ RAND_MAX \&\& random $<0.99999744 *$ RAND_MAX) steps $=18$;

else if (random $>=0.99999744 *$ RAND_MAX \&\& random $<0.99999872 *$ RAND_MAX) steps $=19$;

else if (random $>=0.99999872 *$ RAND_MAX \&\& random $<=1 *$ RAND_MAX) steps $=20$;

\}

else if (lamda $==6)$

\{

if (random $>=0 \& \&$ random $<0.00255641 *$ RAND_MAX) steps $=0$;

else if (random $>=0.00690897 *$ RAND_MAX $\& \&$ random $<0.01753077 *$ RAND_MAX) steps $=1$;

else if (random $>=0.01753077 *$ RAND_MAX \& \& random $<0.06192436 *$ RAND_MAX) steps $=2$;

else if (random $>=0.06192436 *$ RAND_MAX \&\& random $<0.15124615 *$ RAND_MAX) steps $=3$;

else if (random $>=0.15124615 *$ RAND_MAX \& \& random $<0.28500000 *$ RAND_MAX) steps $=4$;

else if (random $>=0.28500000 *$ RAND_MAX \&\& random $<0.44495513 *$ RAND_MAX) steps $=5$;

else if (random $>=0.44495513 *$ RAND_MAX \&\& random $<0.60494231 *$ RAND_MAX) steps $=6$;

else if (random $>=0.60494231 *$ RAND_MAX \&\& random $<0.74322051 *$ RAND_MAX) steps $=7$;

else if (random $>=0.74322051 *$ RAND_MAX \&\& random $<0.84681410 *$ RAND_MAX) steps $=8$;

else if (random $>=0.84681410 *$ RAND_MAX \&\& random $<0.91555256 *$ RAND_MAX) steps $=9$;

else if (random $>=0.91555256 *$ RAND_MAX \&\& random $<0.95706410 *$ RAND_MAX) steps $=10$;

else if (random $>=0.95706410 *$ RAND_MAX \&\& random $<0.97981538 *$ RAND_MAX) steps $=11$;

else if (random $>=0.97981538 *$ RAND_MAX \&\& random $<0.99120128 *$ RAND_MAX) steps $=12$;

else if (random $>=0.99120128 *$ RAND_MAX \&\& random $<0.99640000 *$ RAND_MAX) steps $=13$;

else if (random $>=0.99640000 *$ RAND_MAX \&\& random $<0.99865000 *$ RAND_MAX) steps $=14$;

else if (random $>=0.99865000 *$ RAND_MAX \&\& random $<0.99952308 *$ RAND_MAX) 
steps $=15$;

else if (random $>=0.99952308 *$ RAND_MAX \&\& random $<0.99984744 *$ RAND_MAX) steps $=16$;

else if (random $>=0.99984744 *$ RAND_MAX \&\& random $<0.99995513 *$ RAND_MAX) steps $=17$;

else if (random $>=0.99995513 *$ RAND_MAX \&\& random $<0.99998590 *$ RAND_MAX) steps $=18$;

else if (random $>=0.99998590 *$ RAND_MAX \&\& random $<0.99999487 *$ RAND_MAX) steps $=19$;

else if (random $>=0.99999487 *$ RAND_MAX \&\& random $<1 *$ RAND_MAX) steps $=20$;

\}

else if $($ lamda $==8)$

\{

if (random $>=0 \& \&$ random $<0.00029359 *$ RAND_MAX) steps $=0$;

else if (random $>=0.00029359 *$ RAND_MAX $\& \&$ random $<0.00314359 *$ RAND_MAX) steps $=1$;

else if (random $>=0.00314359 *$ RAND_MAX $\& \&$ random $<0.01382692 *$ RAND_MAX) steps $=2$;

else if (random $>=0.01382692 *$ RAND_MAX $\& \&$ random $<0.04210769 *$ RAND_MAX) steps $=3$;

else if (random $>=0.04210769 *$ RAND_MAX \&\& random $<0.09962692 *$ RAND_MAX) steps $=4$;

else if (random $>=0.09962692 *$ RAND_MAX \& \& random $<0.19146538 *$ RAND_MAX) steps $=5$;

else if (random $>=0.19146538 *$ RAND_MAX \&\& random $<0.31265385 *$ RAND_MAX) steps $=6$;

else if (random $>=0.31265385 *$ RAND_MAX \& \& random $<0.45267949 *$ RAND_MAX) steps $=7$;

else if (random $>=0.45267949 *$ RAND_MAX \&\& random $<0.59227821 *$ RAND_MAX) steps $=8$;

else if (random $>=0.59227821 *$ RAND_MAX \& \& random $<0.71677564 *$ RAND_MAX) steps $=9$;

else if (random $>=0.71677564 *$ RAND_MAX \&\& random $<0.81542436 *$ RAND_MAX) steps $=10$;

else if (random $>=0.81542436 *$ RAND_MAX \&\& random $<0.88796410 *$ RAND_MAX) steps $=11$;

else if (random $>=0.88796410 *$ RAND_MAX \&\& random $<0.93629231 *$ RAND_MAX) steps $=12$;

else if (random $>=0.93629231 *$ RAND_MAX \&\& random $<0.96601026 *$ RAND_MAX) steps $=13$;

else if (random $>=0.96601026 *$ RAND_MAX \&\& random $<0.98285385 *$ RAND_MAX) steps $=14$;

else if (random $>=0.98285385 *$ RAND_MAX \&\& random $<0.99180641 *$ RAND_MAX) steps $=15$;

else if (random $>=0.99180641 *$ RAND_MAX \&\& random $<0.99634359 *$ RAND_MAX) steps $=16$;

else if (random $>=0.99634359 *$ RAND_MAX \&\& random $<0.99842051 *$ RAND_MAX) steps $=17$;

else if (random $>=0.99842051 *$ RAND_MAX \&\& random $<0.99937179 *$ RAND_MAX) 


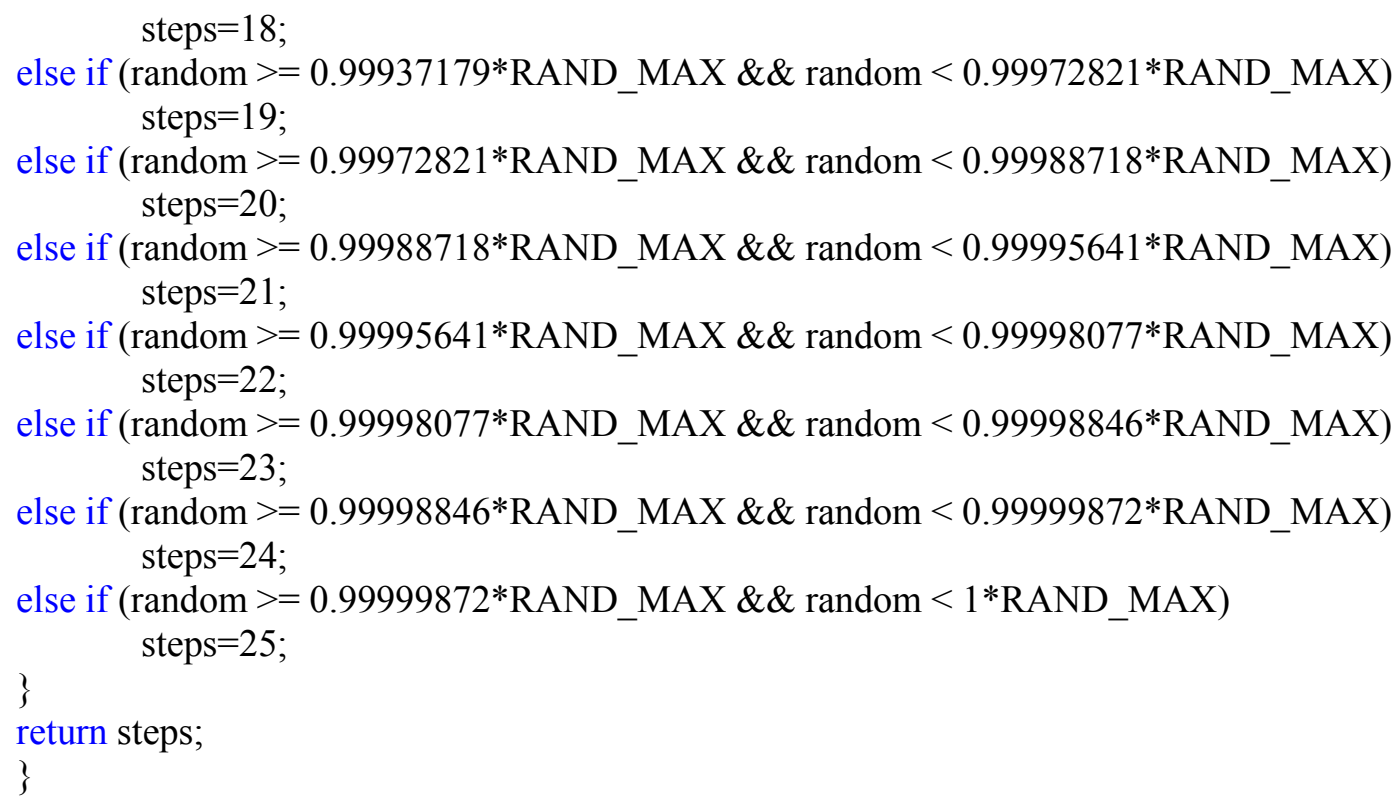




\section{VITA}

Markos Leggas was born in Athens, Greece on September 22, 1968. He immigrated to the United States in 1984 and finished his junior and senior year of high school. He entered Union University in 1986 and in June 1991, he received the degree of Bachelor of Science in Chemistry. The following October he started working as a chemist at Schering-Plough Healthcare Products in Memphis, Tennessee. In August 1996 he entered the University of Tennessee, Memphis and in June 1999 received a Master of Science degree in Biomedical Engineering.

He is presently continuing his education at the University of Tennessee, Memphis in the Ph.D. program in Biomedical Engineering. 